\title{
A Bayesian Framework for Adaptive Selection, Calibration, and Validation of Coarse-Grained Models of Atomistic Systems
}

\author{
Kathryn Farrell, J. Tinsley Oden, and Danial Faghihi \\ Institute for Computational Engineering and Sciences \\ The University of Texas at Austin \\ kfarrell@ices.utexas.edu, oden@ices.utexas.edu, danial@ices.utexas.edu
}

September 24, 2014

\begin{abstract}
A general adaptive modeling algorithm for selection and validation of coarsegrained models of atomistic systems is presented. A Bayesian framework is developed to address uncertainties in parameters, data, and model selection. Algorithms for computing output sensitivities to parameter variances, model evidence and posterior model plausibilities for given data, and for computing what are referred to as Occam Categories in reference to a rough measure of model simplicity, make up components of the overall approach. Computational results are provided for representative applications.
\end{abstract}

Keywords: Coarse graining models, Bayesian inference, output sensitivities, model plausibility, model validation.

\section{Introduction}

The subject of model validation in the presence of uncertainties - in observational data, in model parameters, and uncertainties in the generally subjective process of selecting the model itself - lies at the very foundations of the scientific method. Scientific knowledge is acquired though observations of physical events and though the development of scientific hypotheses on the causes of the events. The former requires the acquisition of relevant observational data and the latter are the consequences of inductive logic leading to mathematical and computational models, the validity of which must be tested against experiments or observations.

Here we consider issues of model selection and validation in connection with coarse graining of atomic systems: the creation of models of atomistic systems by 
aggregating clusters of atoms into "beads" or "super atoms" so as to dramatically reduce the number of degrees of freedom and also to extend time scales in which physical quantities of interest can be observed. The overriding issues in developing coarse-grained $(\mathrm{CG})$ models are how accurately they approximate key quantities of interest captured by the all-atom (AA) system; that is, is the CG model valid in some sense, and, more fundamentally, how does one select the CG model itself to faithfully represent relevant properties of the AA model? It is important that inherent uncertainties encountered in each step of the model validation and selection process must also be taken into account. In this study, a Bayesian framework is developed to address these issues.

A large literature on CG models exists, going back over a half century as early versions of CG approximations appeared in the 1940's, with more general approaches appearing decades later in computer implementations (e.g. [16, 19]). The recent survey of Noid [34] of CG models of bio-molecular system cites almost 600 works and the survey of Li et al. [29] with over 400 references attests to the great interest in CG methodologies. Among methods proposed for constructing CG models using AA-model data, we mention force-matching methods [13, 2225], the so-called "multiscale coarse-graining" methods of Izvekov and Voth et al. $[20,21,25,53,57]$ which were shown to be "physically consistent" in $[33,35,36]$, the iterative Boltzmann inversion methods of [46] in which CG parameters are chosen to fit specific probability distributions in AA systems, the Reverse Monte Carlo (RMC) method [30-32], the Conditional Reverse Work (CRW) method [6, 7], and the methods of Shell et al. based on parameter selections that minimize the relative entropics of CG and AA systems (e.g. [9, 10, 52]).

The present work, which builds on the general framework for model validation and selection advanced in [15] and [40], follows somewhat different arguments than earlier approaches, as it attempts to make specific the processes of model selection and validation in the presence of uncertainties.

Our general approach approach toward selecting and validating CG models can be described as an adaptive modeling paradigm that employs Bayesian and information theoretics and consists of the following five components:

I Parametric Model Classes: A family of possible parametric model classes with unknown parameters $\boldsymbol{\theta}$ defining a particular CG interatomic potential.

II Parameter Sensitivity Analysis: A parameter sensitivity analysis performed over the entire set of $\mathcal{M}$ models. Here we employ a variance-based sensitivity analysis that computes the sensitivity of a representative output function (here the total potential energy) due to variations in parameters using Monte-Carlo samples of parameter probability densities, retaining only parameters that significantly influence the output.

III Occam Categories: We partition the surviving models into categories numbered according to the number of unknown parameters in each model. Here we equate 
the number of parameters to the simplicity of the model in the spirit of Occam's Razor, knowing that this is not always a precise designation.

IV Model Plausibilities and Parameters Calibration: For models in a given set, we compute the Bayesian posterior plausibility of each model for given AA data in a calibration scenario. The use of model plausibilities in selecting CG models is discussed in $[14,15,41]$. The calibration scenario is viewed as an initial characterization of probability densities of parameters for models of representative molecules that are to be tested in one or more validation tests. Upon calculating plausibilities, the most plausible is selected.

V Validation Tests: One or more validation tests are designed to test the accuracy with which the most plausible model from IV predicts CG observables. A validation criterion must be specified to judge if the prediction is adequate and that, therefore, the model is not invalid ("valid"). If the model is valid, the process is terminated. The validated model is then used to solve the forward problem for the target quantities of interest (QoI) and the uncertainty in the QoI is quantified using standard measures.

Following this introduction, we present full details on each of the above steps of the model selection and validation process and give examples of applications to representative classes of models in which each component of the algorithm is addressed.

\section{The Problem Setting}

\subsection{The AA Model}

We begin with the general process of creating CG models designed to deliver approximations of properties of a "ground-truth" all-atom (AA) model, the properties of which are assumed to be known (the force field, parameters, etc.). The AA model thus provides synthetic observational data for calibrating and validating the CG model for specific observables and quantities of interest (QoI). For specificity, the behavior of the atomic system is assumed to be captured through molecular dynamics (MD) simulations, implemented using a hardened and generally accepted MD code. Also for simplicity, and without loss in generality, we restrict attentions to configurational energies of canonical ensembles of AA systems. In the present investigation, we employ LAMMPS [44]. A force field (the functional form of the potential energy) of the general form calibrated in $[27,28]$ is employed,

$$
u(\mathbf{r})=V_{\text {bond }}(\mathbf{r})+V_{\text {angle }}(\mathbf{r})+V_{\text {dihedral }}(\mathbf{r})+V_{\text {non-bonded }}(\mathbf{r})+V_{\text {coulomb }}(\mathbf{r}),
$$


where $\mathbf{r}=\mathbf{r}^{n}=\left\{\mathbf{r}_{1}, \mathbf{r}_{2}, \ldots, \mathbf{r}_{n}\right\}$ is the vector of atomic coordinates in an $n$-atom system, and, as typical examples of energy characterizations,

$$
\begin{aligned}
V_{\text {bond }}(\mathbf{r}) & =\sum_{i=1}^{N_{b}} \frac{1}{2} k_{r i}\left(r_{i}-r_{0 i}\right)^{2}, \\
V_{\text {angle }}(\mathbf{r}) & =\sum_{i=1}^{N_{a}} \frac{1}{2} k_{\theta i}\left(\theta_{i}-\theta_{0 i}\right)^{2}, \\
V_{\text {dihedral }}(\mathbf{r}) & =\sum_{i=1}^{N_{d}} \sum_{n=1}^{4} \frac{V_{n} i}{2}\left[1+(-1)^{n-1} \cos \left(n \phi_{i}\right)\right],
\end{aligned}
$$

and, typically, the non-bonded energy is of a Lennard-Jones form,

$$
\begin{aligned}
V_{\text {non-bonded }}(\mathbf{r}) & =\sum_{i=1}^{N_{n b}} \sum_{j>i} 4 \epsilon_{i j}\left(\left(\frac{\sigma_{i j}}{r_{i j}}\right)^{\alpha}-\left(\frac{\sigma_{i j}}{r_{i j}}\right)^{\beta}\right), \quad r_{i j} \leq r_{c}, \\
V_{\text {columb }}(\mathbf{r}) & =\sum_{i=1}^{N_{q}-1} \sum_{j>i}^{N_{q}} 4 \epsilon_{0} \frac{q_{i} q_{j}}{r_{i j}}
\end{aligned}
$$

Here $N_{b}, N_{a}, \ldots, N_{q}$ are the numbers of representative interaction types, $(\alpha, \beta)$ is the Lennard-Jones (LJ) pair (typically $\alpha=12, \beta=6$ ), $r_{c}$ is the cut-off distance for non-bonded interactions, $\varepsilon_{i j}=\sqrt{\varepsilon_{i i} \varepsilon_{j j}}, \sigma_{i j}=\sqrt{\sigma_{i i} \sigma_{j j}}, \epsilon_{0}$ is the universal permitivity constant, and $r_{i j}=\left\|\mathbf{r}_{i}-\mathbf{r}_{j}\right\|$. Force field constants $k_{r i}, k_{\theta i}, \varepsilon_{i i}$, and $V_{n i}$; geometric constants $r_{0 i}, \theta_{0 i}$, and $\sigma_{i i}$; and the Coulomb charges at atoms $i$ and $j, q_{i}$ and $q_{j}$, respectively, are the force field parameters. Many alternative forms of the potential $u(\mathbf{r})$ can be found in the literature and are used in other well-established MD codes (e.g. $[8,11,56])$. Our general approach can accommodate any of the common potentials used in MD calibrations. Generally, we consider charge-neutral systems so that $V_{\text {coulomb }}=0$.

To provide further focus on modeling issues, we generally confine ourselves to the setting of canonical ensembles, also called NVT ensembles, in which the number of atoms, volume, and temperature are fixed. For such ensembles, the probability with which configurations of the system are accessed is given by the Boltzmann distribution,

$$
\rho(\omega)=\exp \{-\beta u(\omega)+a \beta\}
$$

where $\omega=\mathbf{r}^{n}, \beta=k_{B} T$, ( $k_{B}$ being the Boltzmann constant, $T$ the absolute temperature, and $a$ a normalization constant). The observables of the system are often taken to be ensemble averages of phase functions $q(\omega)$, denoted

$$
\langle q\rangle=\int_{\Gamma_{A A}} \rho(\omega) q(\omega) d \omega,
$$


$\Gamma_{A A}$ being the AA phase-space. Note that observables may also be distributions $\pi(q(\omega))$. In general, we invoke the ergodic hypothesis and evaluate (4) for given $q(\omega)$ using samples drawn from MD calculations with, for example, the Nosé-Hoover thermostat employed to maintain constant temperature.

\subsection{The CG Models}

It has been understood for decades that the enormous size and complexity of AA models needed to capture events at length and time scales relevant in countless applications in material science, nanomanufacturing, biomedicine, and other areas, far exceeds the capacity of today's computing systems or even those envisioned many years into the future. Thus, methods for reducing the number of degrees of freedom and lengthening simulation time periods by aggregating atoms into coarse-grained molecular models is viewed as a necessary approach to studies of most atomistic simulations.

Let $\mathbf{R}^{N}=\left\{\mathbf{R}_{1}, \mathbf{R}_{2}, \ldots, \mathbf{R}_{N}\right\}$ denote the molecule ("bead") coordinates in a CG representation of an AA system which contains $n$ atoms. It is convenient to regard each configuration $\mathbf{R}^{N}$ as the image of a set of atom coordinates $\mathbf{r}^{n} \in$ $\Gamma_{A A}$ under a map $G$, and for simplicity in notation, we write $\mathbf{r}^{n}=\omega$ and $\mathbf{R}^{n}=$ $G(\omega)$. We elaborate on the structure and definition of $G$ in the next section. As we are considering only conformational energies, the resulting Hamiltonian of the CG system is,

$$
H\left(\mathbf{R}^{N} ; \boldsymbol{\theta}\right)=H(G(\omega) ; \boldsymbol{\theta})=U(G(\omega) ; \boldsymbol{\theta})
$$

where $U(.,$.$) is the potential energy and \boldsymbol{\theta}$ is the vector of parameters for the particular CG representation. The general form of $U(.,$.$) is identical to (1) with \mathbf{r}$ replaced by the $N$ CG coordinates $\mathbf{R}^{N}$, and,

$$
\boldsymbol{\theta}=\left\{K_{R i}, R_{0 i}, K_{\theta i}, \theta_{0 i}, V_{n i}, \epsilon_{i i}, \sigma_{i i}\right\}
$$

are the unknown parameters of the CG model. Our goal is to calculate an acceptable approximation of the QoI (4) using the CG model,

$$
Q(\boldsymbol{\theta})=\langle q(\boldsymbol{\theta})\rangle=\int_{\Gamma_{C G}} \rho_{C G}(G(\omega) ; \boldsymbol{\theta}) q(G(\omega)) d G(\omega),
$$

$\Gamma_{C G}$ being the reduced phase space in the $\mathrm{CG}$ model and $\rho_{C G}$ is the CG Boltzmann distribution,

$$
\rho_{C G}=\exp \{-\beta U(G(\omega) ; \boldsymbol{\theta})+\beta A(\boldsymbol{\theta})\} .
$$

Thus, we again consider a canonical ensemble with N, V, T fixed. Note that since $\boldsymbol{\theta}$ will be a random variable, stochastically determined as discussed in Section $3, Q(\boldsymbol{\theta})$ is actually a probably density function. Further details are given in Section 3.5.

The significant problems with these approximations are obvious: 1) the number $N$ of CG particles is not precisely specified (i.e. the choice of the map $G$ is not 


\section{BAYESIAN FRAMEWORK FOR MODEL VALIDATION UNDER}

well-defined); 2) once specified, the choice and structure of the interaction potentials $V_{\text {bond }}, V_{\text {angle }}, \ldots, V_{\text {non-bonded }}$ of (2) is unspecified; 3 ) importantly, the parameters $\boldsymbol{\theta}$ are unknown for every choice of $N$ and every set of potentials chosen; and 4) each step is met with uncertainties in the AA data, the parameters, and the model output.

\subsection{Parametric Model Classes}

The general setting is illustrated in Figure 1, where several possible CG approximations are declared, each with a dependent AA-to-CG map $G$, and for each choice, different choices of intermolecular force fields $V$, such as those in (1) are possible. For each choice of $G$ (equivalently $N$ ) and potentials $V$, the model parameters $\boldsymbol{\theta}$ are unknown.

The idea of mapping a set of atoms in the AA system into a CG bead is standard in literature on coarse-graining methods, but the actual definition of such maps can involve technicalities. For example, " $G: \mathrm{AA} \rightarrow \mathrm{CG}$ " may be a multi-valued relation (not a function) on coordinate pairs $\left(\mathbf{r}_{n}, \mathbf{R}_{N}\right)$ in which an AA coordinate vector $\mathbf{r}_{\alpha}, 1 \leq \alpha \leq n$, is identified with a CG coordinate vector $\mathbf{R}_{A}, 1 \leq A \leq N$, the coordinate labels $\alpha$ being members of an index set $\mathcal{J}_{A}$. In the case of bonded CG systems, for example, an AA coordinate vector can be associated with more than one index set. The CG coordinate vector $\mathbf{R}_{A}$ may be located at any convenient point within the bead " $A$ ", but often is chosen as the mass center $\left(\mathbf{R}_{A}=\sum_{\alpha \in \mathcal{J}_{A}} m_{\alpha} \mathbf{r}_{\alpha} / \sum_{\alpha \in \mathcal{J}_{A}} m_{\alpha}\right.$, it being understood that fractions of atomic masses are distributed to beads sharing atoms). The relationship between AA and CG coordinate vector labels can be expressed as simply $\mathbf{R}_{A}=G_{A}^{\cdot \alpha} \mathbf{r}_{\alpha}$, sum on $\alpha$, where $G_{A}^{\cdot \alpha}$ is the Boolean array, $G_{A}^{\cdot \alpha}=1$ if $\alpha \in \mathcal{J}_{A}$ and $G_{A}^{\cdot \alpha}=0$ if otherwise. We generally write symbolically, $\mathbf{R}^{N}=G\left(\mathbf{r}^{n}\right)=G(\omega),\left(\omega=\mathbf{r}^{n}\right)$.

The CG process can thus lead to a large family $\mathcal{M}$ of possible model classes $\mathcal{M}_{i}$ each with a different AA-to-CG map $G$,

$$
\begin{aligned}
\mathcal{M} & =\left\{\mathcal{M}_{1}, \mathcal{M}_{2}, \ldots, \mathcal{M}_{k}\right\} \\
\mathcal{M}_{i} & =\left\{\mathcal{P}_{i 1}\left(\boldsymbol{\theta}_{i 1}\right), \mathcal{P}_{i 2}\left(\boldsymbol{\theta}_{i 2}\right), \ldots, \mathcal{P}_{i m}\left(\boldsymbol{\theta}_{i m}\right)\right\}, \quad i=1,2, \ldots, k .
\end{aligned}
$$

For simplicity in notation, we pick one class $\mathcal{M}\left(=\mathcal{M}_{1}\right)$ and denote its members $\left\{\mathcal{P}_{1}\left(\boldsymbol{\theta}_{1}\right), \mathcal{P}_{2}\left(\boldsymbol{\theta}_{2}\right), \ldots, \mathcal{P}_{m}\left(\boldsymbol{\theta}_{m}\right)\right\}$.

\section{Bayesian Framework for Model Validation Under Un- certainty}

To provide an appropriate context for developments in Sections 4 and 5, we outline a Bayesian framework for validation (see, e.g. $[2,14,15,38-40]$ ). Our goal is the following: given $A A$ data in one or more calibration scenarios (defined below) find the most plausible model $\mathcal{P}_{j}\left(\boldsymbol{\theta}_{j}\right) \in \mathcal{M}$, in a parametric model class $\mathcal{M}_{i} \in \mathcal{M}$, and (simultaneously) determine probability density functions (pdfs) representing the 


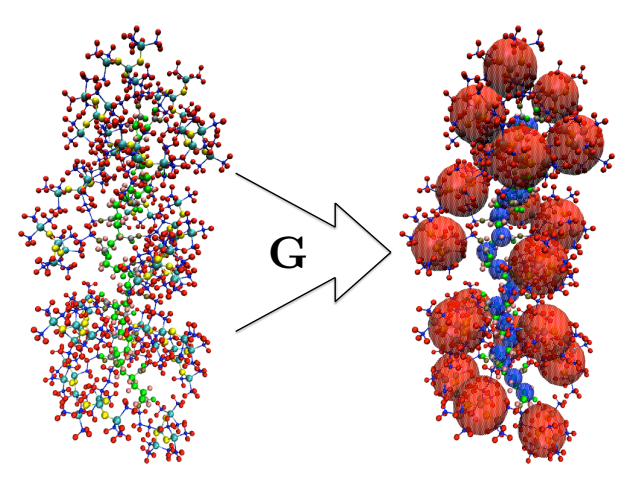

(a)

$$
\mathcal{M}_{k} \text { : }
$$

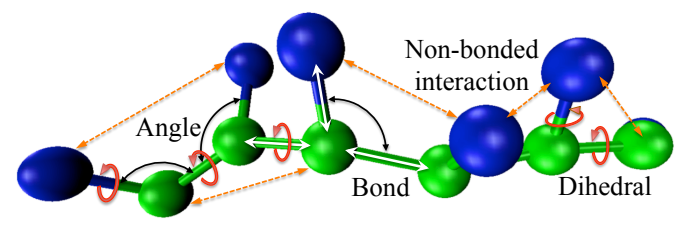

$\mathcal{P}_{k 1}$

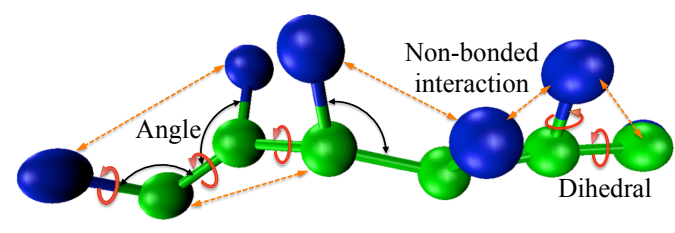

$\mathcal{P}_{k 3}$

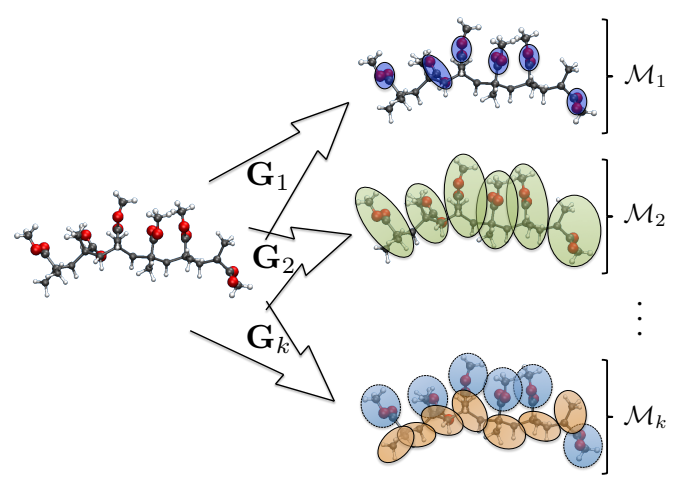

(b)

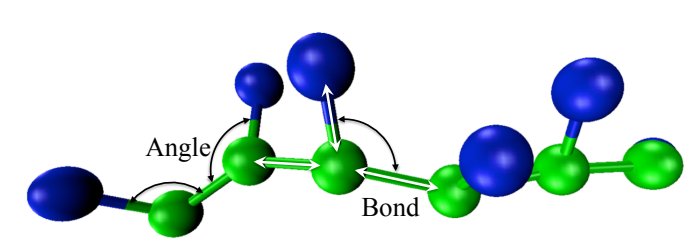

$\mathcal{P}_{k 2}$

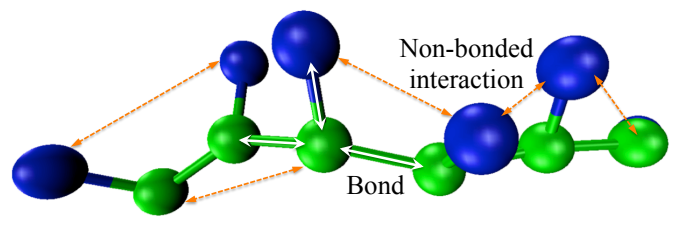

$\mathcal{P}_{k m}$

(c)

Figure 1: (a) Coarse-graining of an all-atom system; (b) different choices of AAto-CG map $G$; (c) different choices of intermolecular force fields $V$, for each map $G$.

uncertainty in parameters of the most plausibe model in the class. Then, having statistically calibrated a model deemed most plausible in $\mathcal{M}$, perform validation tests in validation scenarios to determine if the accuracy with which the model predicts validation observable is sufficient to claim the model is "valid" (not invalid) for the purpose for which it was intended, it being assumed that the validation observables depict in some reasonable way the quantity of interest.

We note that a fundamental tenant in the art and science of model validation is that the notion of validity of a model is only meaningful in connection with specific quantities of interest; a model may be deemed valid for predicting one QoI but invalid for another [40]. Philosophically, the QoI is not an observable; it is data that 
must be extrapolated beyond the range of data accessible in validation tests. Thus, a challenge in designing validation tests is to choose subjectively observables that represent the QoI. These concepts are illustrated in example calculations to follow.

\subsection{Scenarios}

The idea of calibration, validation, and prediction scenarios is described in several papers $[2,14,15,38-40]$ and is a useful concept for orchestrating the process of model validation. Scenarios are basically restricted solution domains of mathematical models of physical events, the calibration scenarios generally representing those appearing in simple component tests designed to calibrate parameters, while the validation scenarios correspond to more complex subsystem experiments designed to test the accuracy of model prediction against carefully selected observables that hopefully depict key quantities of interest that reside as extrapolations of the observable data. The prediction scenario corresponds to the full forward problem in which a validated model is used to predict quantities of interest.

In general, the calibration, validation, and prediction scenarios are conceptualized as levels in a "prediction pyramid" (see, e.g. [1, 2, 14, 15, 38-40]). The calibration scenario $S_{c}$ is represented by the base of the pyramid where the model accesses calibration data $\mathbf{D}_{c}$. Traveling upwards, the validation scenario $S_{v}$ accesses validation observables represented by validation data $\mathbf{D}_{v}$, and $S_{p}$, the full-system prediction scenario, is at the peak of the pyramid where the non-observable QoI resides.

\subsection{Output Sensitivity}

In many cases, one or more model parameters may be ignored or eliminated in parametric models if key model outputs are not affected by changes in the parameter values. One approach, which we adopt in this work, is to employ variance-based methods in which the sensitivity pattern of a model is described by decomposing the variance $V(Y)$ of an output function $Y=Y\left(\theta_{1}, \theta_{2}, \cdots, \theta_{k}\right)$ into contributions from each of the parameters $[12,54,55]$. By using Monte Carlo samples of each parameter, total sensitivity indices are estimated via,

$$
S_{T_{i}}=\frac{E_{\boldsymbol{\theta}_{\sim i}}\left(V_{X_{i}}\left(Y \mid \boldsymbol{\theta}_{\sim i}\right)\right)}{V(Y)}
$$

Here, $\boldsymbol{\theta}_{\sim i}$ is the input vector excluding the $i$ th component $\theta_{i}, V_{X_{i}}\left(Y \mid \boldsymbol{\theta}_{\sim i}\right)$ is the variance due to $\theta_{i}$ of the output produced by $\boldsymbol{\theta}_{\sim i}$ and $E_{\boldsymbol{\theta}_{\sim i}}$ is the conditional expectation of the model given $\boldsymbol{\theta}_{\sim i}$. The indices $S_{T_{i}}$ provide a measure of the sensitivity of each input factor on the total output variance. Further details are given in Appendix A.

A more qualitative route to computing sensitivities is to construct scatterplots [49]. In this method, the parameters are randomly sampled and the output 
function $Y$ is computed as mentioned above. For each parameter, samples are compared directly to the value of $Y$. A parameter is considered to be important if a clear correlation between the parameter's value and the output value can be seen in the scatterplot patterns. Two examples are discussed in the next subsection.

We assert that when applied to the Bayesian framework discussed in this section, the sensitivity analysis should be performed in both the calibration and prediction scenarios. When implemented in the prediction scenario, the output function should be the QoI. The parameters shown to be important are those that have the most influence on the QoI and should therefore be included in the model calibration. When implemented in the calibration scenario, the output function should be the same observable used as calibration data in the Bayesian update (14). Then, not only are the important parameters influential to the output, we can say that the observable will inform the parameters during calibration. Both points are critical: the parameters should influence the QoI and they need to be informed (updated) by the calibration observable.

\subsubsection{Example: Polyethylene Chain}

In order to reduce the parameter space dimension, quantitative sensitivity analysis is conducted on the CG system to determine the relative level of importance of each parameter to the others. As the first sensitivity scenario, a single chain with 12 beads, with two carbon atoms per bead, is considered in a constant volume and temperature (i.e. $T=100 \mathrm{~K}$ ) canonical ensemble. The chain is deformed by applying a force to the bead at one end while the other end is constrained. As model output $Y$, we take the difference between average potential energies before and after deformation. That is,

$$
Y(\boldsymbol{\theta})=\Delta\langle U\rangle=\left\langle U\left(\boldsymbol{\theta}, G_{1}\right)\right\rangle-\left\langle U\left(\boldsymbol{\theta}, G_{0}\right)\right\rangle,
$$

where $G_{0}$ is the initial conformation of the chain and $G_{1}$ is the conformation after deformation.

With respect to this observable, the scatterplots for some of the parameters are shown in Fig. 2. These plots were obtained by sampling the parameters from given distributions and computing the model output at each sample point. The distributions assumed are

$$
\begin{array}{rlrl}
r_{0} & \sim \mathcal{U}(0.1,5) ; & & K_{r} \sim \mathcal{U}(0.1,90) ; \\
\theta_{0} \sim \mathcal{U}(80,180) ; & K_{\theta} & \sim \mathcal{U}(0,15) ; \\
V_{1} & \sim \mathcal{U}(-1,1) ; & & V_{2} \sim \mathcal{U}(-1,1) ; \\
V_{2} & \sim \mathcal{U}(-1,1) ; & & V_{4} \sim \mathcal{U}(-1,1) ; \\
\sigma & \sim \mathcal{U}(0.05,7) ; & & \epsilon \mathcal{U}(0.01,5) .
\end{array}
$$

Total sensitivity indices (10) are also estimated using the method described in Section A.3. The result for each parameter is shown in Fig 3. It can be seen 


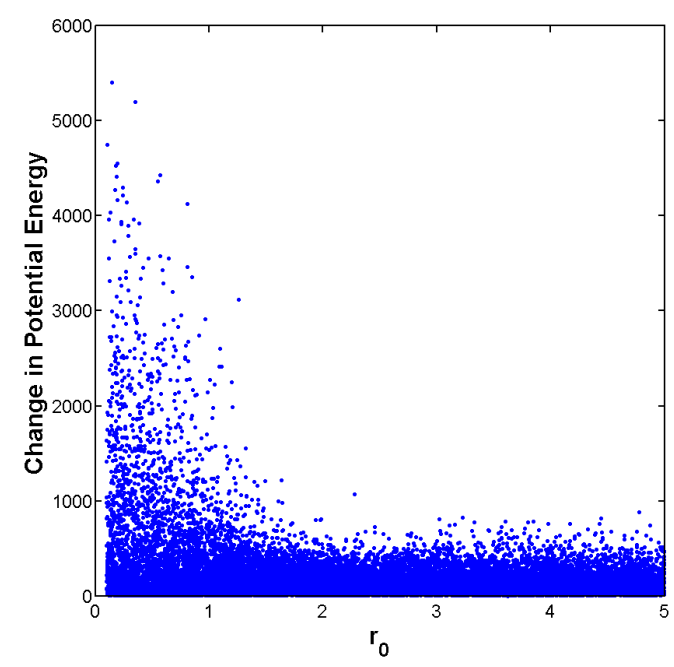

(a)

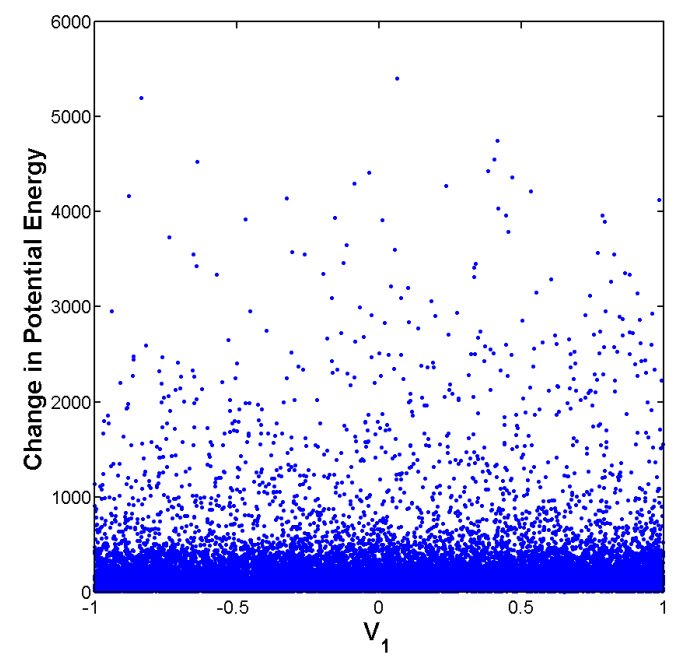

(c)

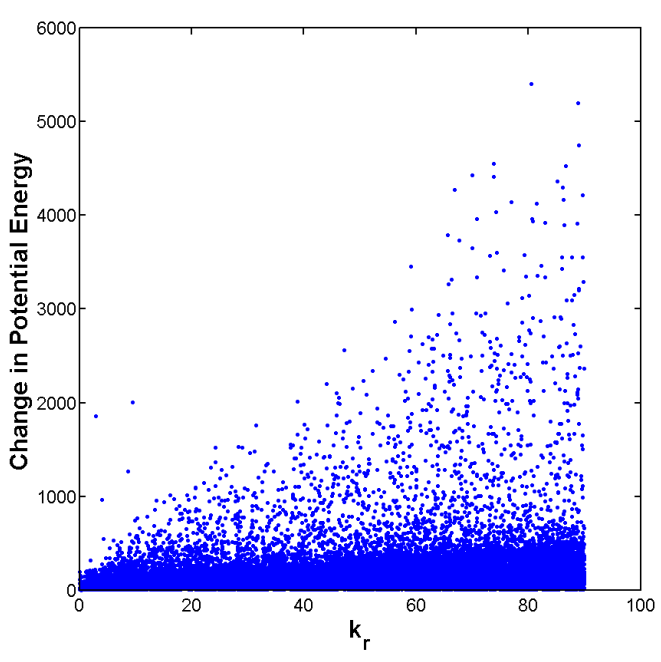

(b)

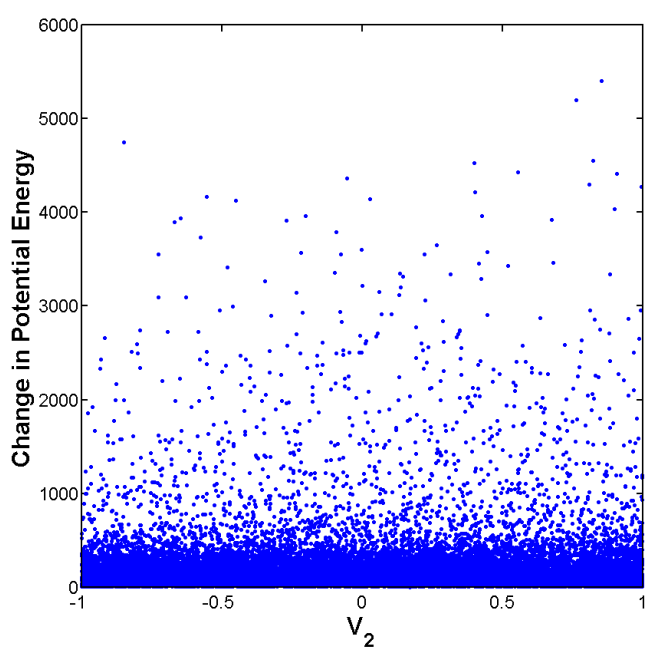

(d)

Figure 2: Scatterplots illustrating a qualitative sensitivity analysis for the polyethylene chain using 40,000 Monte Carlo samples of the parameter space. The observable is the change in potential energy as the chain is deformed.

qualitatively from scatterplots of Fig. 2 and quantitatively from total sensitivity indices of Fig. 3 that the dihedral parameters, $V_{n}, n=1, \ldots, 4$, do not have a significant effect on the value of the change in potential energy as the polyethylene chain is deformed. 


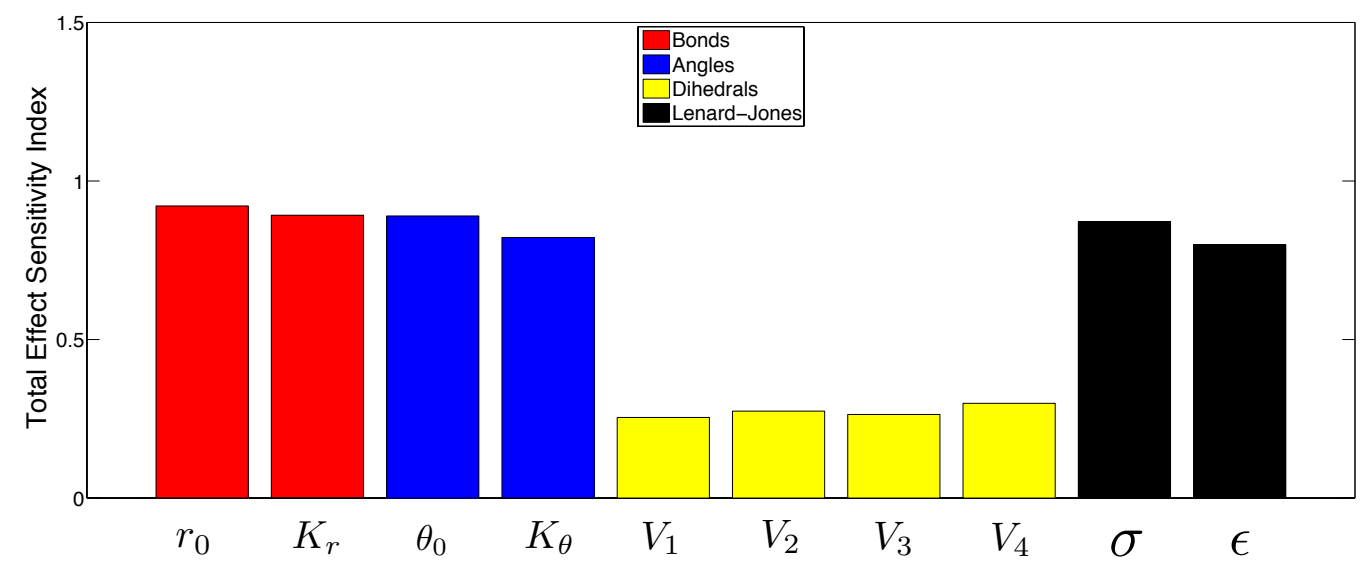

Figure 3: Bar chart illustrating the results of the sensitivity analysis using total sensitivity indices for the deformation of the polyethylene chain using 20,000 Monte Carlo samples of the parameter space.

To confirm this result, we compute the same output function $Y=\Delta\langle U\rangle$, now considering two sets of parameters, one in which the dihedral parameters are sampled and used in the calculation and the other in which the dihedral parameters are excluded. The kernel density estimate (KDE) for each case is presented in Fig. 4. The minimal difference between the two KDEs indicates the insignificant effect of the dihedral parameters on the change in the average potential energy as the chain is deformed. Therefore, we conclude that these parameters may be excluded from the calibration process.

\subsubsection{Example: Polyethylene System}

A natural question that arises is whether sensitivity tests performed at the calibration scenario remain relevant for the full system or for validation scenarios. To explore this issue, we consider a more complex polyethylene system closer to a prediction scenario consisting of a complex system of polymer chains shown in Fig. 5. A configuration of a polyethylene system initially contained in a volume of size $10 \times 10 \times 10 \mathrm{~nm}$ and under biaxial deformation (along the $x$ and $y$ directions) is considered. The initial chain structure is created using a method similar to those previously developed using Monte Carlo self-avoiding random walks [5]. The initial structure shown in Fig. 5 contains 500 chains with $10 \mathrm{CG}$ beads per chain. Before applying the deformation, an equilibration sequence is performed on the initial structure, following the steps presented [18]. The equlibrated polyethylene system is then deformed under biaxial tensile strain applied at a constant strain rate. The mechanical response of the system can be characterized by the variation of the Second Piola-Kirchhoff stress against the Lagrangian Green strain in each 


\section{BAYESIAN FRAMEWORK FOR MODEL VALIDATION UNDER}

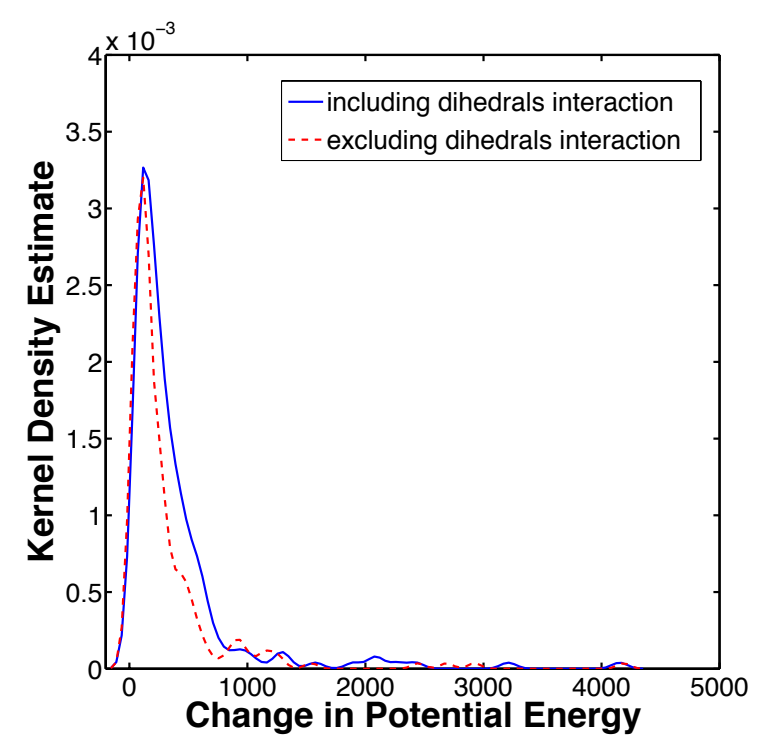

Figure 4: Comparison between the kernel density estimates of the change in potential energy resulting from the deformation of a polyethylene chain for the cases when dihedral parameters are included and excluded from calculations. Results show 200 Monte Carlo samples of the parameter space.

direction and, correspondingly, against the Helmholtz free energy, here equivalent to the strain-energy density function $W$. As model output $Y$, we take the values of $W$ at $\lambda_{x}=1.3$, where $\lambda_{x}$ (i.e. the principal stretch) is defined as the ratio of the length of the deformed simulation box along the $x$-axis to the undeformed length measured at the end of the equilibration step. That is,

$$
Y(\boldsymbol{\theta})=W\left(\lambda_{x}=1.3\right) .
$$

Scatterplots generated for bond length $r_{0}$ and angle $\theta_{0}$ as well as two dihedral coefficients, $V_{3}$ and $V_{4}$, are shown in Fig. 6. The estimated total effect sensitivity indices for all parameters are presented in Fig. 7. Comparing these results to those in Section 3.2.1, we conclude that the effect of bond length and angle are more pronounced in the larger scale polyethylene system, while the dihedral interactions between the beads remain weaker. The latter observation confirms the assumptions made in $[42,43]$ for simulating large scale amorphous polyethylene and illustrates that, indeed, dihedrals can be excluded from the calibration.

\subsection{Calibration}

Let $\mathbf{D}_{c}$ be the vector of observational data supplied by the AA model for a calibration scenario $\mathbf{D}_{c}$ being a vector in a space $\mathcal{D}$ of observational data. If $d: \Theta \rightarrow \mathcal{D}$ is 
the parameter-to-observation map provided by model $\mathcal{P}$, and if the discrepancy $\varepsilon$ between observational data and model prediction is additive and represented by a random variable with density $p$, then the density

$$
\pi\left(\mathbf{D}_{c} \mid \boldsymbol{\theta}\right)=p\left(\mathbf{D}_{c}-\mathbf{d}(\boldsymbol{\theta})\right)
$$

is the likelihood function, the conditional probability density of the data given parameters $\boldsymbol{\theta}$. The error $\varepsilon$ represents the sum of the observational noise and error due to model inadequacy $[14,40]$. The density $p$ is often assumed to be Gaussian with covariance matrix defined by parameters that are added to the list of model parameter $\boldsymbol{\theta}$ and are determined by the calibration tests.

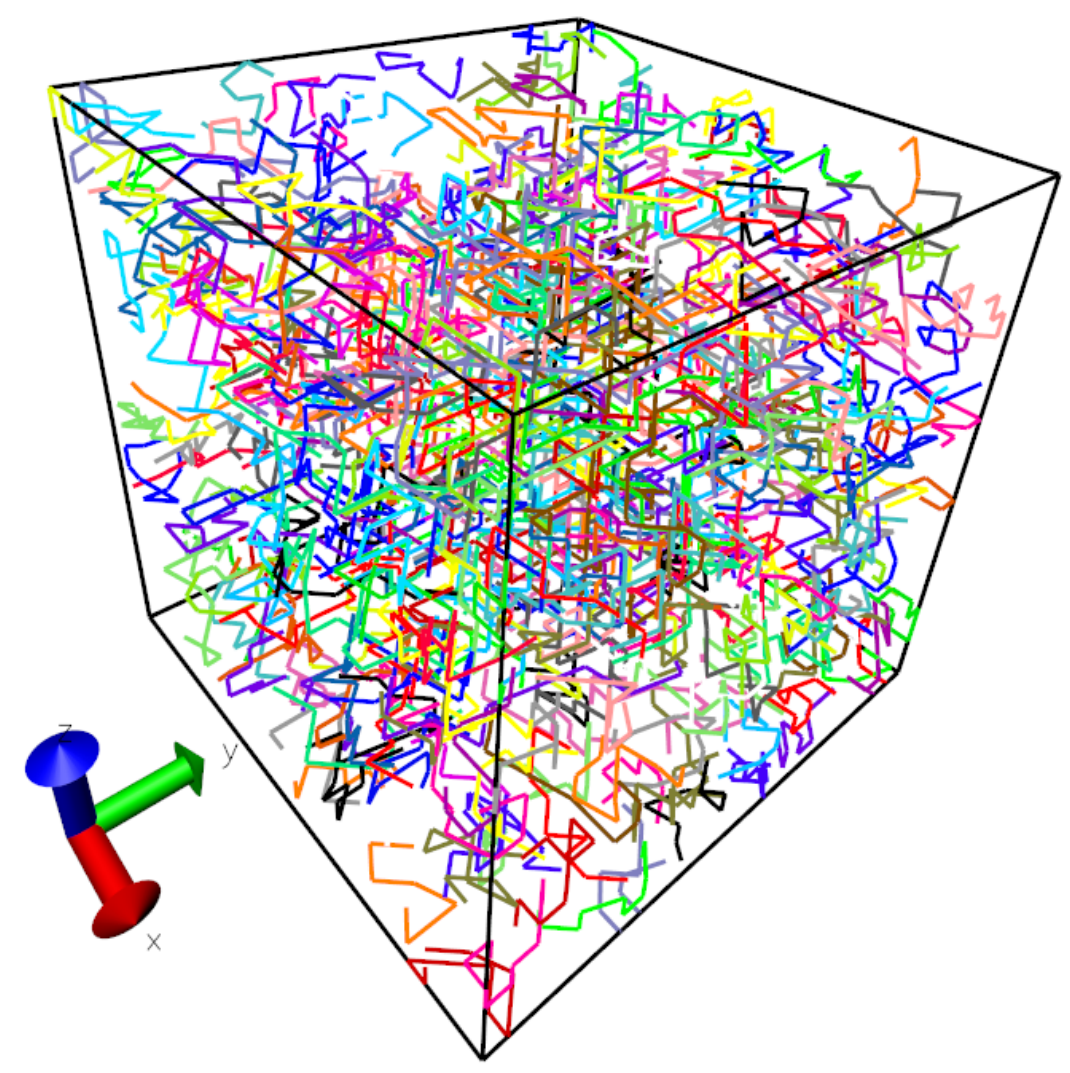

Figure 5: Sensitivity analysis scenario close to the prediction scenario of a polyethylene system. The configuration is generated using the Monte Carlo self-avoiding random walk algorithm within a cube of dimensions $10 \times 10 \times 10 \mathrm{~nm}$. The colored lines represent different chains (total number of chains $=500$, chain length $=10 \mathrm{CG}$ beads). 


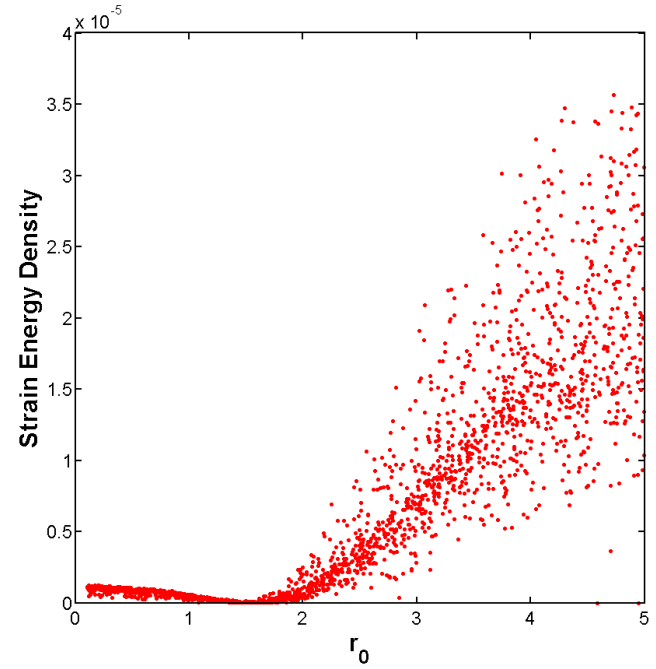

(a)

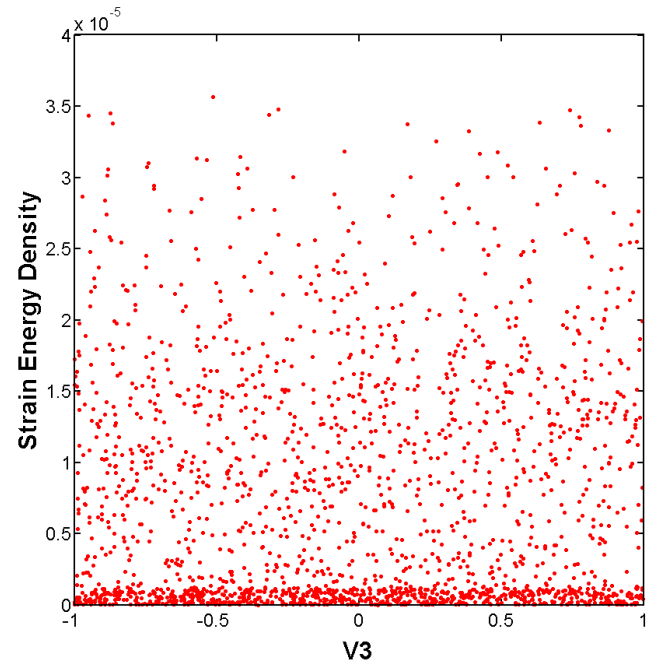

(c)

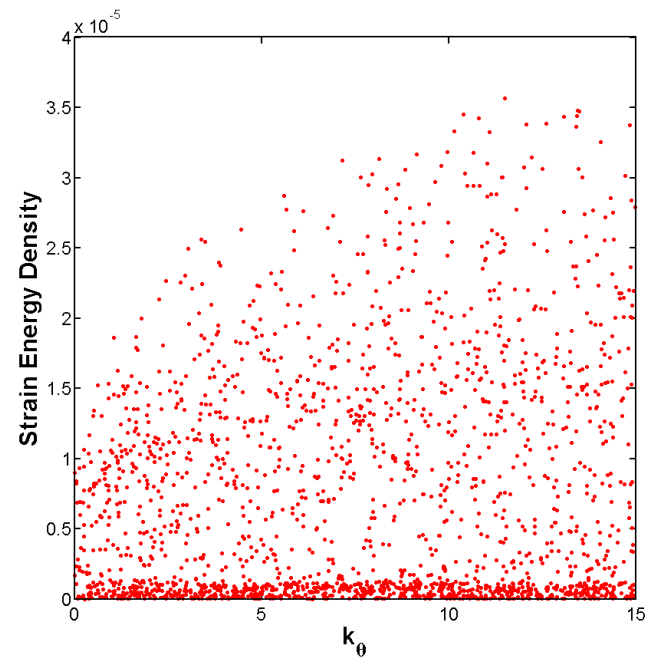

(b)

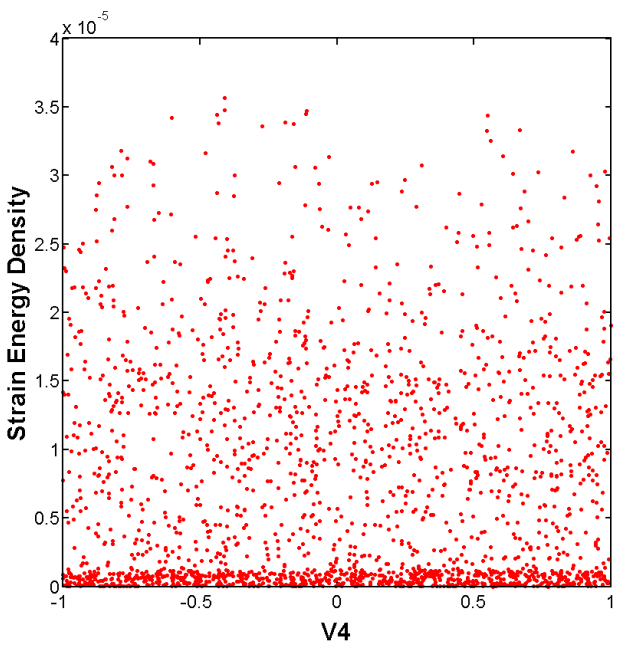

(d)

Figure 6: Scatterplots illustrating a qualitative sensitivity analysis for the prediction scenario of the polyethylene system using 1000 Monte Carlo samples of the parameter space. The observable is the value of the strain-energy density function $W$ at the deformation corresponding to $\lambda_{x}=1.3$.

The posterior probability density is given by Bayes's rule,

$$
\pi\left(\boldsymbol{\theta} \mid \mathbf{D}_{c}\right)=\frac{\pi\left(\mathbf{D}_{c} \mid \boldsymbol{\theta}\right) \pi(\boldsymbol{\theta})}{\pi\left(\mathbf{D}_{c}\right)}
$$




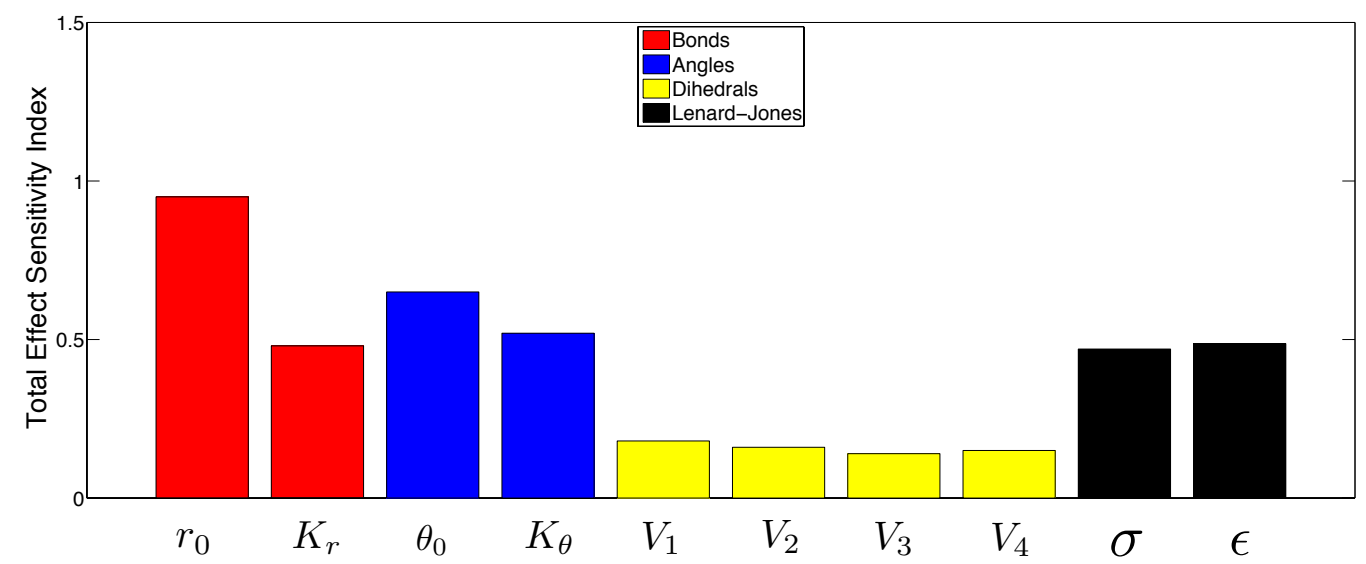

Figure 7: Bar chart illustrating the results of the sensitivity analysis using total sensitivity indices for the deformation of the prediction scenario of the polyethylene system using 1000 Monte Carlo samples of the parameter space.

where $\pi(\boldsymbol{\theta})$ is the prior, representing prior information on $\boldsymbol{\theta}$ and $\pi\left(\mathbf{D}_{c}\right)$ is the evidence,

$$
\pi\left(\mathbf{D}_{c}\right)=\int_{\Theta} \pi\left(\mathbf{D}_{c} \mid \boldsymbol{\theta}\right) \pi(\boldsymbol{\theta}) d \boldsymbol{\theta}
$$

$\Theta$ being the parameter space for model $\mathcal{P}$.

When prior information about parameters can be inferred from data from the AA model, the prior can be computed using the maximum entropy method advocated by Jaynes [26]. In [15] it is demonstrated that useful prior probabilities can be derived from AA data. Given suitable priors and the likelihood function, the actual posterior distribution (14) is determined by implementing any one of several robust sampling algorithms. In the examples described later, we employ a MCMC (Markov Chain Monte Carlo) algorithm available in the software package QUESO $[45]$.

\subsection{Model Plausibility}

Returning to Bayes's rule (14), and recognizing explicitly that we are calibrating a single model $\mathcal{P}_{j}$ among a set of possible models $\mathcal{M}$, we rewrite the rule in an equivalent way that recognizes probabilities conditioned on the choices $\mathcal{P}_{j}$ and $\mathcal{M}$,

$$
\pi\left(\boldsymbol{\theta}_{j} \mid \mathbf{D}_{c}, \mathcal{P}_{j}, \mathcal{M}\right)=\frac{\pi\left(\mathbf{D}_{c} \mid \boldsymbol{\theta}_{j}, \mathcal{P}_{j}, \mathcal{M}\right) \pi\left(\boldsymbol{\theta} \mid \mathcal{P}_{j}, \mathcal{M}\right)}{\pi\left(\mathbf{D}_{c} \mid \mathcal{P}_{j}, \mathcal{M}\right)}, \quad 1 \leq j \leq m
$$

The marginalization of the numerator on the right-hand side is the model evidence,

$$
\pi\left(\mathbf{D}_{c} \mid \mathcal{P}_{j}, \mathcal{M}\right)=\int_{\Theta} \pi\left(\mathbf{D}_{c} \mid \boldsymbol{\theta}, \mathcal{P}_{j}, \mathcal{M}\right) \pi\left(\boldsymbol{\theta} \mid \mathcal{P}_{j}, \mathcal{M}\right) d \boldsymbol{\theta}
$$


The model evidence can be regarded as a likelihood density in a higher form of Bayes's rule, where we compute the posterior model plausibility,

$$
\rho_{j}=\pi\left(\mathcal{P}_{j} \mid \mathbf{D}_{c}, \mathcal{M}\right)=\frac{\pi\left(\mathbf{D}_{c} \mid \mathcal{P}_{j}, \mathcal{M}\right) \pi\left(\mathcal{P}_{j} \mid \mathcal{M}\right)}{\pi\left(\mathbf{D}_{c} \mid \mathcal{M}\right)}, \quad 1 \leq j \leq m
$$

where,

$$
\sum_{j=1}^{m} \rho_{j}=1 .
$$

The model(s) in class $\mathcal{M}$ with plausibilities $\rho_{j}$ closest to one are deemed the most plausible for given data $\mathbf{D}_{c}$. The use of model plausibilies as a basis for model selection has been advocated $[4,37,40,41]$ and used successfully in selecting CG models in [15].

\subsection{Validation}

With the calibration posterior $\pi\left(\boldsymbol{\theta} \mid \mathbf{D}_{c}\right)$ for the model judged at this point to be the most plausible in hand, we compute the posterior for a validation scenario with validation data $\mathbf{D}_{v}$ :

$$
\pi\left(\boldsymbol{\theta} \mid \mathbf{D}_{v}, \mathbf{D}_{c}\right)=\frac{\pi\left(\mathbf{D}_{v} \mid \boldsymbol{\theta}, \mathbf{D}_{c}\right) \pi\left(\boldsymbol{\theta} \mid \mathbf{D}_{c}\right)}{\pi\left(\mathbf{D}_{v} \mid \mathbf{D}_{c}\right)}
$$

Thus, the validation posterior in (20) represents an update of the calibrated parameters obtained using new data $\mathbf{D}_{v}$ designed to reflect the best representation of the QoI possible in the validation scenario (see, e.g. $[15,40]$ ). While not always necessary, it is possible at this point to compute the information gain,

$$
I\left(\pi\left(\boldsymbol{\theta} \mid \mathbf{D}_{v}, \mathbf{D}_{c}\right), \pi\left(\boldsymbol{\theta} \mid \mathbf{D}_{c}\right)\right)=\int_{\mathbf{D}_{v}} D_{K L}\left(\pi\left(\boldsymbol{\theta} \mid \mathbf{D}_{v}, \mathbf{D}_{c}\right) \| \pi\left(\boldsymbol{\theta} \mid \mathbf{D}_{c}\right)\right) \pi\left(\mathbf{D}_{v}\right) d \mathbf{D}_{v}
$$

as a measure of the value of the validation-test information over that embodied in the calibrations. Here, $D_{K L}(\cdot \| \cdot)$ is the Kullback-Leibler divergence between two distributions $p$ and $q$ defined to be

$$
D_{K L}(p \| q)=\int p(y) \log \frac{p(y)}{q(y)} d y .
$$

With the updated parameters determined by (20), we now solve the forward problem for the validation observable and compute the accuracy of the CG prediction. It is important to note that the observable computed by the CG model depends on the parameters $\boldsymbol{\theta}$. Since the parameters are random variables, so are the observables. Therefore, to solve the forward problem, the validation posterior $\pi\left(\boldsymbol{\theta} \mid \mathbf{D}_{v}, \mathbf{D}_{c}\right)$ is stochastically sampled and a pdf $\pi(U(\boldsymbol{\theta}, G))$ of the observable, for example the potential energy, is produced. 
If the target observable, such as the AA potential energy, is a probability distribution, $\pi\left(u_{A A}\right)$, a natural measure of the accuracy of the CG approximation is the Kullback-Leibler divergence,

$$
\gamma=D_{K L}\left(\pi\left(u_{A A}\right) \| \pi(U(\boldsymbol{\theta}, G))\right)=\int_{\Gamma_{v}} \pi\left(u_{A A}(\omega)\right) \log \frac{\pi\left(u_{A A}(\omega)\right)}{\pi(U(\boldsymbol{\theta}, G(\omega)))} d \omega,
$$

where $\Gamma_{v}$ is the reduced phase space available in the validation scenario. If instead, the target observable is a scalar, usually an ensemble average such as $\left\langle u_{A A}\right\rangle$, the corresponding CG value must be found by taking the expected value,

$$
\langle U\rangle=\mathbb{E}_{\pi_{v}}[\langle U(\boldsymbol{\theta}, G)\rangle]=\int_{\Theta} \pi(U(\boldsymbol{\theta}, G)) \pi\left(\boldsymbol{\theta} \mid \mathbf{D}_{v}, \mathbf{D}_{c}\right) d \boldsymbol{\theta}
$$

Then the results may be compared in a Euclidean metric,

$$
\gamma=\left|\left\langle u_{A A}\right\rangle-\langle U\rangle\right|
$$

For a preset tolerance $\gamma_{t o l}$, we declare the model valid (technically, "not invalid") if

$$
\gamma \leq \gamma_{t o l}
$$

Otherwise, the model is invalid and we return to the class of models and choose the most plausible in the next level of Occam categories, and repeat the calibration, validation process. This is discussed in detail in Section 4.

\section{The Occam-Plausibility Algorithm}

Occam's razor is the principle that states that among competing theories that lead to the same prediction, the one that relies on the fewest assumptions is the best. In particular, when choosing among a set of models, the simplest valid model is the best choice. Exactly how to quantify simplicity has been a subject of debate for centuries. Here, the simplicity of a model is assumed to be determined by the number of parameters in the model: a model with two parameters is simpler than a model with three. This is in part due to the complexity and computational cost of solving the inverse problem that yields these parameters. Furthermore, the notion of model validity is determined by a Bayesian validation test. With this principle in mind, the following Occam-Plausibility (OP) algorithm is proposed. An example with full detail is given in Section 5 .

\section{Initialization}

The algorithm begins when a set $\mathcal{M}$ of possible models is identified. Each model $\mathcal{P}_{j}$ in this set has its own parameters, $\boldsymbol{\theta}_{j}$ so that

$$
\mathcal{M}=\left\{\mathcal{P}_{1}\left(\boldsymbol{\theta}_{1}\right), \ldots, \mathcal{P}_{m}\left(\boldsymbol{\theta}_{m}\right)\right\}
$$


Generally, the models within $\mathcal{M}$ will be closely related; there may be two or more models that have some of the same parameters. For example, in the case of coarsegraining, $\mathcal{M}$ is created by choosing an AA-to-CG map (or several of such maps) and tabulating the possible combinations of types of interactions, as detailed in Section 5 below.

\section{Sensitivity Analysis}

By computing model sensitivities, as described in Section 3.2, a new set of possible models may be defined

$$
\overline{\mathcal{M}}=\left\{\overline{\mathcal{P}}_{1}\left(\overline{\boldsymbol{\theta}}_{1}\right), \ldots, \overline{\mathcal{P}}_{l}\left(\overline{\boldsymbol{\theta}}_{l}\right)\right\}, \quad l \leq m,
$$

where $\overline{\mathcal{P}}_{j}$ are the models in $\mathcal{M}$ that contain only parameters to which the model output is sensitive and models containing output-insensitive parameters have been eliminated.

\section{Occam Step}

After eliminating models that contain insensitive parameters to produce the new set of models $\overline{\mathcal{M}}$, the remaining models can be separated into categories based on the number of model parameters. The models with the least parameters belong to Category 1, Category 2 models are slightly more complex and so on. See, for example, Table 1.

In the Occam step, the set

$$
\mathcal{M}^{*}=\left\{\mathcal{P}_{1}^{*}\left(\boldsymbol{\theta}_{1}^{*}\right), \ldots, \mathcal{P}_{k}^{*}\left(\boldsymbol{\theta}_{k}^{*}\right)\right\}
$$

is created such that all of the models $\mathcal{P}_{i}^{*}, 1 \leq i \leq k$ are Category 1 . That is, $\mathcal{M}^{*}$ contains those models in $\overline{\mathcal{M}}$ that have the smallest number of parameters. We begin the calibration and validation cycle (discussed in Section 3) with the simplest model, thus adhering to Occam's Razor.

\section{Calibration and Plausibility Steps}

All of the models in $\mathcal{M}^{*}$ are calibrated via Bayes' rule (16) and their plausibilities (18) are calculated. The most plausible model $\mathcal{P}_{j}^{*}$ may be identified such that

$$
\rho_{i}^{*} \leq \rho_{j}^{*}, \quad i=1, \ldots, k, \quad i \neq j .
$$

Only $\mathcal{P}_{j}^{*}$ will be subjected to the validation test(s). 


\section{Validation Step}

The parameters of the most plausible model are updated via Bayes' rule (20), where the observational data $\mathbf{D}_{v}$ comes from the validation scenario. These updated parameters are then used in a forward problem and measured for accuracy via (23) or (25), with validity determined by (26). If the model $\mathcal{P}_{j}^{*}$ is deemed valid, it is suitable for use in the prediction scenario; otherwise, the model is invalid. Once a valid model is identified, the $(\mathrm{OP})$ algorithm is complete. Otherwise the algorithm proceeds to the iterative Occam step.

\section{Iteration}

If $\mathcal{P}_{j}^{*}$ is invalid, $\mathcal{M}^{*}$ is redefined so as to contain models of the next category. If $\mathcal{P}_{j}^{*}$ is Category 1 , the new $\mathcal{M}^{*}$ would contain Category 2 models, and so on. One additional constraint that may be imposed is to mandate that all of the models in the new $\mathcal{M}^{*}$ contain all of the parameters in $\mathcal{P}_{j}^{*}$ so that they build on the information learned about the preference the system has about model parameters.

If this iteration has been repeated enough times so that $\mathcal{P}_{j}^{*}$ already belongs to the highest category of models in $\overline{\mathcal{M}}$, the modeler must define a new set of models $\mathcal{M}$, conduct a new sensitivity analysis, and iterate through the algorithm until a valid model is found. A flowchart summarizing the full OP algorithm is shown in Figure 8.

\section{An Example Application}

We return to the application of coarse graining to polyethylene to illustrate the OP Algorithm. Recall that in this example, to coarse grain polyethylene, two carbon atoms and their accompanying hydrogen atoms are aggregated into a single coarse-grained particle. The coarse-grained system of polyethylene has one type of particle, yielding only one type of bond, one type of angle, and one type of dihedral interaction.

\section{Initialization}

As mentioned previously, the set of coarse-grained models $\mathcal{M}=\left\{\mathcal{P}_{1}\left(\boldsymbol{\theta}_{1}\right), \ldots\right.$, $\left.\mathcal{P}_{23}\left(\boldsymbol{\theta}_{23}\right)\right\}$ is created by tabulating the possible combinations of interactions, which are listed in Table 1. Note that the Lennard-Jones 9-6 potential has been added as an alternative to the Lennard-Jones 12-6. The LJ 9-6 is a softer potential to account for the empty space that results from grouping atoms into a single sphere, which is an idealization of the CG particle. Furthermore, previous studies have indicated that an additive constant parameter should be added to the potential [15]. This parameter, $A$, represents the potential energy lost due to the coarse graining process and can be thought of as the internal bead energy. 


\section{START}

Identify a set of possible models

$$
\mathcal{M}=\left\{\mathcal{P}_{1}\left(\boldsymbol{\theta}_{1}\right), \ldots, \mathcal{P}_{m}\left(\boldsymbol{\theta}_{m}\right)\right\}
$$

\section{SENSITIVITY ANALYSIS}

Eliminate models with parameters to

which the model output is insensitive $\mathcal{M} \mapsto \overline{\mathcal{M}}=\left\{\overline{\mathcal{P}}_{1}\left(\overline{\boldsymbol{\theta}}_{1}\right), \ldots, \overline{\mathcal{P}}_{l}\left(\overline{\boldsymbol{\theta}}_{l}\right)\right\}, \quad l \leq m$

$$
\longleftarrow \begin{gathered}
\text { Identify new set of possible } \\
\text { models } \\
\mathcal{M}=\left\{\mathcal{P}_{1}\left(\boldsymbol{\theta}_{1}\right), \ldots, \mathcal{P}_{m^{\prime}}\left(\boldsymbol{\theta}_{m^{\prime}}\right)\right\}
\end{gathered}
$$

\section{$\downarrow$}

OCCAM STEP

Choose model(s) in lowest Occam category

$\mathcal{M}^{*}=\left\{\mathcal{P}_{1}^{*}\left(\boldsymbol{\theta}_{1}^{*}\right), \ldots, \mathcal{P}_{k}^{*}\left(\boldsymbol{\theta}_{k}^{*}\right)\right\}, \quad k<l$

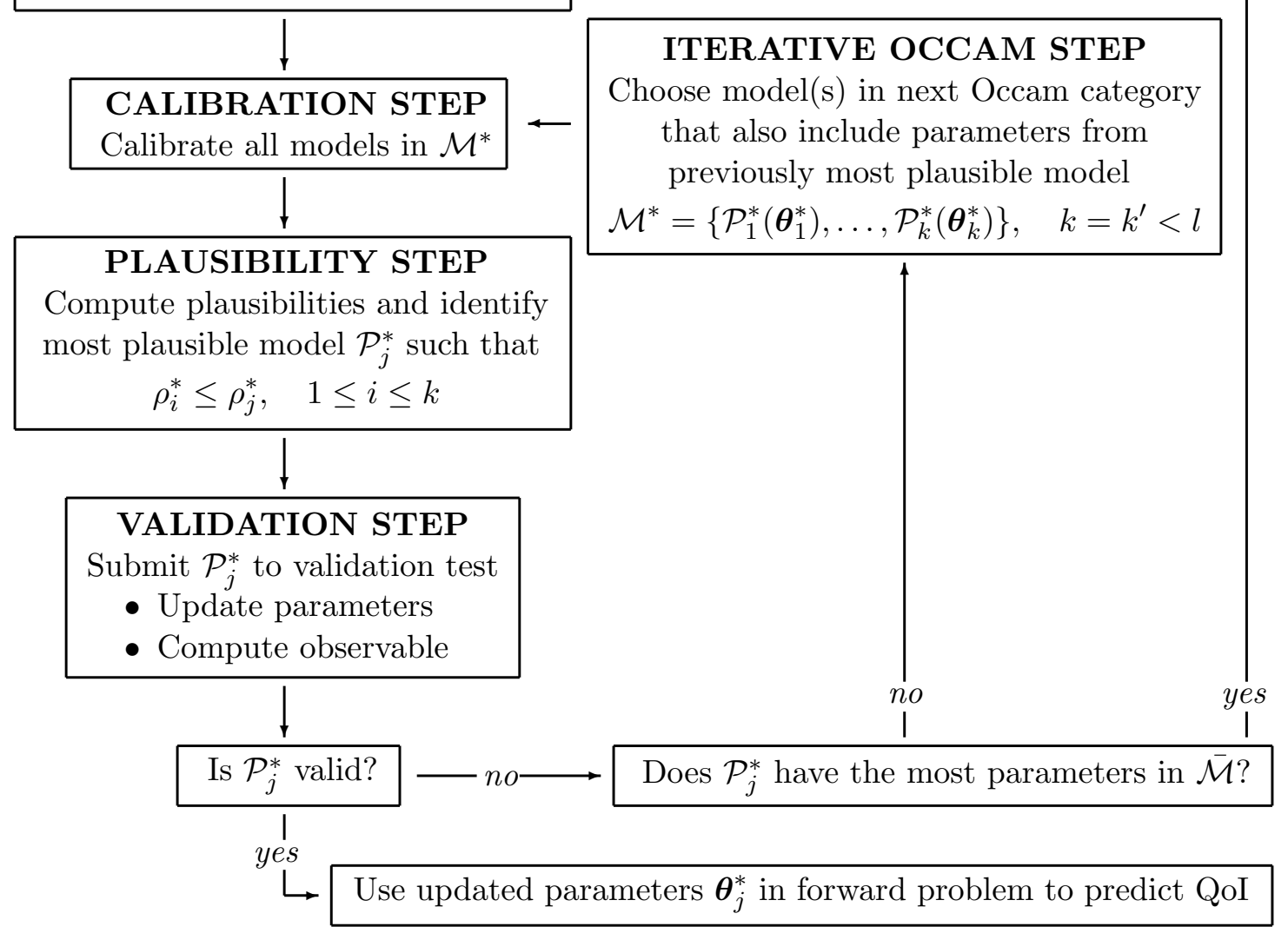

Figure 8: Flowchart of the Occam-Plausibility Algorithm. 


\begin{tabular}{|c|c|c|c|c|c|c|c|}
\hline Model & Bonds & Angles & Dihedrals & LJ 12-6 & LJ 9-6 & Param & Cat. \\
\hline $\mathcal{P}_{1}$ & $\checkmark$ & & & & & 3 & \multirow[t]{4}{*}{1} \\
\hline $\mathcal{P}_{2}$ & & $\checkmark$ & & & & 3 & \\
\hline $\mathcal{P}_{3}$ & & & & $\checkmark$ & & 3 & \\
\hline $\mathcal{P}_{4}$ & & & & & $\checkmark$ & 3 & \\
\hline $\mathcal{P}_{5}$ & $\checkmark$ & $\checkmark$ & & & & 5 & \multirow[t]{6}{*}{2} \\
\hline $\mathcal{P}_{6}$ & $\checkmark$ & & & $\checkmark$ & & 5 & \\
\hline $\mathcal{P}_{7}$ & $\checkmark$ & & & & $\checkmark$ & 5 & \\
\hline $\mathcal{P}_{8}$ & & $\checkmark$ & & $\checkmark$ & & 5 & \\
\hline $\mathcal{P}_{9}$ & & $\checkmark$ & & & $\checkmark$ & 5 & \\
\hline $\mathcal{P}_{10}$ & . & & $\checkmark$ & & & 5 & \\
\hline $\mathcal{P}_{11}$ & $\checkmark$ & $\checkmark$ & & $\checkmark$ & & 7 & \multirow[t]{6}{*}{3} \\
\hline $\mathcal{P}_{12}$ & $\checkmark$ & $\checkmark$ & & & $\checkmark$ & 7 & \\
\hline $\mathcal{P}_{13}$ & $\checkmark$ & & $\checkmark$ & & & 7 & \\
\hline $\mathcal{P}_{14}$ & & $\checkmark$ & $\checkmark$ & & & 7 & \\
\hline $\mathcal{P}_{15}$ & & & $\checkmark$ & $\checkmark$ & & 7 & \\
\hline $\mathcal{P}_{16}$ & & & $\checkmark$ & & $\checkmark$ & 7 & \\
\hline $\mathcal{P}_{17}$ & $\checkmark$ & $\checkmark$ & $\checkmark$ & & & 9 & \multirow[t]{5}{*}{4} \\
\hline $\mathcal{P}_{18}$ & $\checkmark$ & & $\checkmark$ & $\checkmark$ & & 9 & \\
\hline $\mathcal{P}_{19}$ & $\checkmark$ & & $\checkmark$ & & $\checkmark$ & 9 & \\
\hline $\mathcal{P}_{20}$ & & $\checkmark$ & $\checkmark$ & $\checkmark$ & & 9 & \\
\hline $\mathcal{P}_{21}$ & & $\checkmark$ & $\checkmark$ & & $\checkmark$ & 9 & \\
\hline $\mathcal{P}_{22}$ & $\checkmark$ & $\checkmark$ & $\checkmark$ & $\checkmark$ & & 11 & \multirow[t]{2}{*}{5} \\
\hline $\mathcal{P}_{23}$ & $\checkmark$ & $\checkmark$ & $\checkmark$ & & $\checkmark$ & 11 & \\
\hline
\end{tabular}

Table 1: Table of possible CG models created by all combinations of interaction potentials that may be included in the representation of the potential energy along with the number of parameters they contain (including $A$ ) and their Occam categories.

\section{Sensitivity Analysis}

As an observable, we consider the change in the potential energy of a system of polyethylene as a load is applied to it. Data can be collected from the AA version of the system and is compared to the corresponding CG representation, as described in Section 3.2. In the case of calibration, the external force is applied to a chain of 24 carbon atoms and $12 \mathrm{CG}$ particles in the AA and CG systems, respectively. With this in mind, a sensitivity analysis is performed, with the output function $Y$ being the change in potential energy as the chain is stretched. It was shown that of the ten possible parameters, the dihedral parameters are the least influential to the chosen observable. All models with dihedral parameters are consequently removed from the set of possible models, leaving $\overline{\mathcal{M}}=\left\{\overline{\mathcal{P}}_{1}, \ldots, \overline{\mathcal{P}}_{11}\right\}=\left\{\mathcal{P}_{1}, \ldots, \mathcal{P}_{9}, \mathcal{P}_{11}, \mathcal{P}_{12}\right\}$. 


\section{Occam Step}

The remaining models are separated into categories according to the number of model parameters. Category 1 contains models $\overline{\mathcal{P}}_{1}, \overline{\mathcal{P}}_{2}, \overline{\mathcal{P}}_{3}$, and $\overline{\mathcal{P}}_{4}$, each of which have two parameters. Models with four parameters, $\overline{\mathcal{P}}_{5}, \overline{\mathcal{P}}_{6}, \overline{\mathcal{P}}_{7}, \overline{\mathcal{P}}_{8}$, and $\overline{\mathcal{P}}_{9}$, are classified as Category 2. The Category 3 models, $\overline{\mathcal{P}}_{10}$ and $\overline{\mathcal{P}}_{11}$, have six parameters each.

In the first Occam Step, only Category 1 models are considered so that

$$
\mathcal{M}^{*}=\left\{\mathcal{P}_{1}^{*}\left(\boldsymbol{\theta}_{1}^{*}\right), \ldots, \mathcal{P}_{4}^{*}\left(\boldsymbol{\theta}_{4}^{*}\right)\right\},
$$

where $\mathcal{P}_{i}^{*}=\overline{\mathcal{P}}_{i}$. That is, $\mathcal{P}_{1}^{*}$ contains only bonded interactions with $\boldsymbol{\theta}_{1}^{*}=\left\{r_{0}, k_{r}\right\}$; $\mathcal{P}_{2}^{*}$ has only angular interactions, where $\boldsymbol{\theta}_{2}^{*}=\left\{\theta_{0}, k_{\theta}\right\}$; and $\mathcal{P}_{3}^{*}$ and $\mathcal{P}_{4}^{*}$ only account for Lennard-Jones non-bonded interactions with $\boldsymbol{\theta}_{3}^{*}=\boldsymbol{\theta}_{4}^{*}=\{\sigma, \epsilon\}$, though the representations differ slightly as previously mentioned.

\section{Calibration and Plausibility Steps}

Each of these models is calibrated using a Gaussian likelihood distribution with $\mathbf{D}=\left\{D_{i}\right\}_{i=1}^{150}=\left\{u\left(\omega_{i}\right)\right\}_{i=1}^{150}$, where $u\left(\omega_{i}\right)$ is the potential energy of a single hexane molecule in configuration $\omega_{i}$, and $\mathbf{d}(\boldsymbol{\theta})=\left\{U\left(G\left(\omega_{i}\right), \boldsymbol{\theta}\right)\right\}_{i=1}^{150}$, where $U$ is the CG potential energy and $G\left(\omega_{i}\right)$ is the CG representation of the AA configuration $\omega_{i}$. Priors for parameters are computed using the maximum entropy method on statistical data gathered from the AA system, as detailed in the Appendix of [15], yielding the prior distribution functions $\pi\left(\boldsymbol{\theta}_{i}^{*} \mid \mathcal{P}_{i}^{*}, \mathcal{M}\right)$. After all four models are calibrated, the following plausibilities are calculated using (18),

$$
\rho_{1}^{*}=1, \quad \rho_{2}^{*}=0, \quad \rho_{3}^{*}=0, \quad \rho_{4}^{*}=0,
$$

illustrating $\mathcal{P}_{1}^{*}$ is the most (and the only) plausible model.

\section{Validation Step}

Moving into the validation step, the calibration posterior $\pi\left(\boldsymbol{\theta}_{1}^{*} \mid \mathbf{D}, \mathcal{P}_{1}^{*}, \mathcal{M}^{*}\right)$ becomes the prior in the validation scenario, where we consider a canonical ensemble simulation of a single molecule of octadecane. Again, the likelihood is taken to be Gaussian with $\mathbf{D}_{v}=\left\{D_{i}\right\}_{i=1}^{150}=\left\{u\left(\omega_{i}\right)\right\}_{i=1}^{150}$, where $u\left(\omega_{i}\right)$ is the potential energy of the system in configuration $\omega_{i}$, and $\mathbf{d}(\boldsymbol{\theta})=\left\{U\left(G\left(\omega_{i}\right), \boldsymbol{\theta}\right)\right\}_{i=1}^{150}$, where $U$ is the CG potential energy of the corresponding CG configuration $G\left(\omega_{i}\right)$. The parameters for $\mathcal{P}_{1}^{*}$ are updated via $(20)$, and the updated parameters are used to simulate a CG system of octadecane.

For the validation observable, we use the potential energy of the system so that $\pi\left(Q \mid \boldsymbol{\theta}_{1, v}^{*}\right)=\pi\left(U\left(G, \boldsymbol{\theta}_{1, v}^{*}\right)\right)$ if the observable is the entire distribution of the potential energy or, if a scalar is selected, $Q=\left\langle U\left(G, \boldsymbol{\theta}_{1, v}^{*}\right)\right\rangle$. The distributions of 


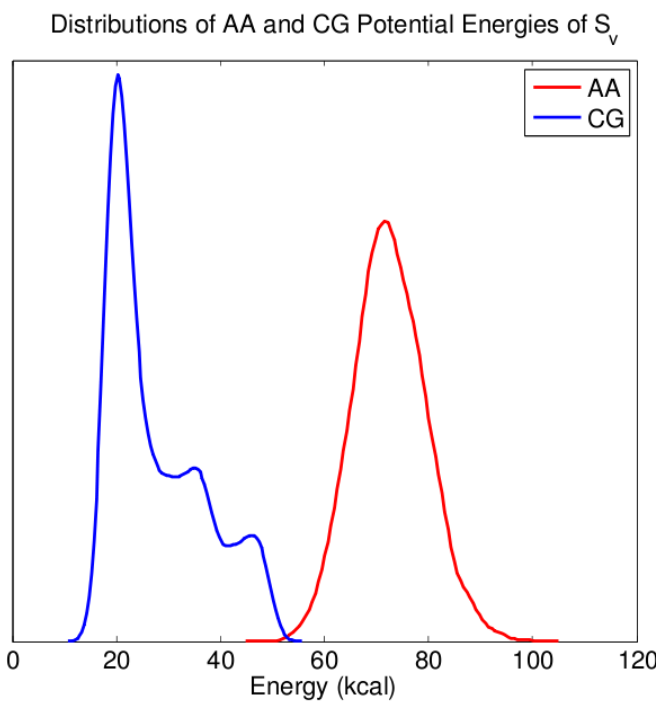

(a)

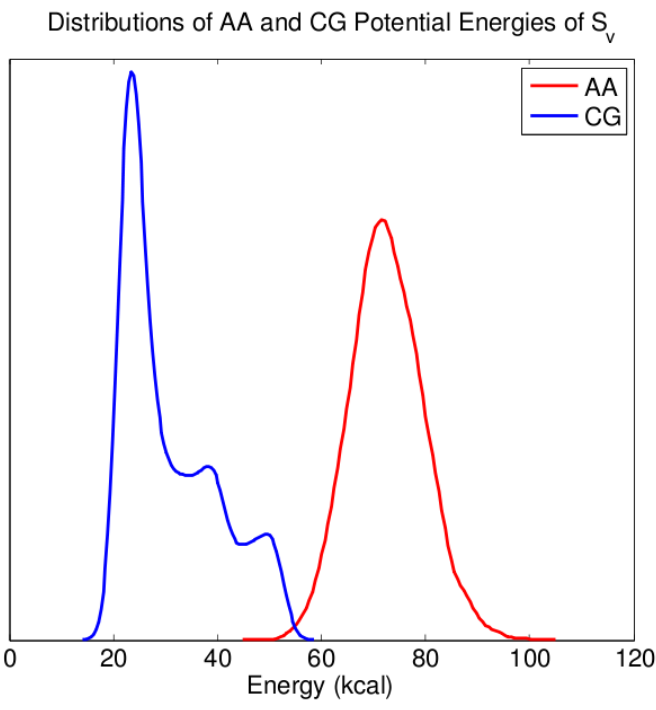

(b)

Figure 9: Comparison of the AA and CG distributions of the validation observable. The CG observables are computed using the validated parameters from the first Occam level (a) and the second Occam level (b). Qualitatively, it can be seen that the CG models are not valid.

the potential energy of the CG and AA systems of octadecane are shown in Fig. 9. From this it can be qualitatively seen that the model is not valid.

Quantitatively, we select the validation criteria for the scalar and pdf observables, respectively, to be

$$
\gamma_{1, t o l}=0.2 Q_{A A}, \quad \gamma_{2, t o l}=0.05 \sigma_{A A}^{2},
$$

where $Q_{A A}=\left\langle u_{A A}\right\rangle$ and $\sigma_{A A}^{2}$ is the variance of $\pi\left(u_{A A}\right)$. Using (23) and (25), we obtain

$$
\gamma_{1}=0.6173 Q_{A A}, \quad \gamma_{2}=0.2435 \sigma_{A A}^{2},
$$

rendering $\mathcal{P}_{1}^{*}$ invalid as $\gamma_{1}>\gamma_{1, t o l}$ and $\gamma_{2}>\gamma_{2, t o l}$.

\section{Iteration}

Moving into the iterative Occam step, a new $\mathcal{M}^{*}$ is defined which contains models in the next category, in this case is Category 2. In addition, we impose the constraint that the models in this set contain the parameters that were included in the previously most-plausible model. That is, models in $\mathcal{M}^{*}$ must contain bond parameters. This constraint creates an algorithm that is closer to hierarchical modeling in which the most plausible model is built upon and improved instead of discarded when invalidated. 
The new $\mathcal{M}^{*}$ is therefore

$$
\mathcal{M}^{*}=\left\{\mathcal{P}_{1}^{*}\left(\boldsymbol{\theta}_{1}^{*}\right), \mathcal{P}_{2}^{*}\left(\boldsymbol{\theta}_{2}^{*}\right), \mathcal{P}_{3}^{*}\left(\boldsymbol{\theta}_{3}^{*}\right)\right\},
$$

where $\mathcal{P}_{1}^{*}=\overline{\mathcal{P}}_{5}$ contains bonded and angular interactions with $\boldsymbol{\theta}_{1}^{*}=\left\{k_{r}, r_{0}, k_{\theta}, \theta_{0}\right\}$, $\mathcal{P}_{2}^{*}=\overline{\mathcal{P}}_{6}$ considers bonded and LJ 12-6 interactions so that $\boldsymbol{\theta}_{2}^{*}=\left\{k_{r}, r_{0}, \sigma, \epsilon\right\}$, and $\mathcal{P}_{3}^{*}=\overline{\mathcal{P}}_{7}$ has bonded and LJ 9-6 interactions with $\boldsymbol{\theta}_{3}^{*}=\left\{k_{r}, r_{0}, \sigma, \epsilon\right\}$.

Considering the same calibration scenario described above, each model in $\mathcal{M}^{*}$ is calibrated. The ensuing plausibility calculations yield,

$$
\rho_{1}^{*}=3.7891 \times 10^{-7}, \quad \rho_{2}^{*}=0.3420, \quad \rho_{3}^{*}=0.6580,
$$

making $\mathcal{P}_{3}^{*}$ the most plausibile model with $\mathcal{P}_{2}^{*}$ close behind. Considering also the same validation scenario, the parameters of $\mathcal{P}_{3}^{*}$ are updated and the same validation test is performed. The resulting distributions of the potential energy are shown in Fig. 9. Using the same observables, $\pi\left(Q \mid \boldsymbol{\theta}_{3, v}^{*}\right)=\pi\left(U\left(G, \boldsymbol{\theta}_{3, v}^{*}\right)\right)$ and $Q=\left\langle U\left(G, \boldsymbol{\theta}_{3, v}^{*}\right)\right\rangle$, we find

$$
\gamma_{1}=0.5731 Q_{A A}, \quad \gamma_{2}=0.2084 \sigma_{A A}^{2}
$$

by (25) and (23), respectively. Thus $\mathcal{P}_{3}^{*}$ is invalid by criteria (33).

We again move through the iterative Occam step, where $\mathcal{M}^{*}$ now contains models of Category 3 that include parameters from the previous $\mathcal{P}_{3}^{*}$. That is,

$$
\mathcal{M}^{*}=\left\{\mathcal{P}_{1}^{*}\left(\boldsymbol{\theta}_{1}^{*}\right), \mathcal{P}_{2}^{*}\left(\boldsymbol{\theta}_{2}^{*}\right)\right\},
$$

where $\mathcal{P}_{1}^{*}=\overline{\mathcal{P}}_{10}$ includes bonded, angular, and LJ 12-6 interactions and $\mathcal{P}_{2}^{*}=\overline{\mathcal{P}}_{11}$ includes bonded, angular, and LJ 9-6 interactions. Both models have the same parameter vector, $\boldsymbol{\theta}_{j}^{*}=\left\{k_{r}, r_{0}, k_{\theta}, \theta_{0}, \sigma, \epsilon\right\}$. Calibration and plausibility calculations show

$$
\rho_{1}^{*}=0.5, \quad \rho_{2}^{*}=0.5,
$$

making both models equally plausible. Performing the validation update and validation test, the results of which are shown in Fig. 10, we calculate

$$
\gamma_{1}=0.1721 Q_{A A}, \quad \gamma_{2}=0.0452 \sigma_{A A}^{2}
$$

Therefore, $\gamma_{1} \leq \gamma_{1, \text { tol }}$ and $\gamma_{2} \leq \gamma_{2, t o l}$, making model $\mathcal{P}_{1}^{*}$ valid according to the validation criteria and observables selected.

\section{Summary and Conclusions}

Bayesian statistical inverse analysis can provide a powerful approach to the calibration and validation of coarse-grained models of atomistic systems, an approach 


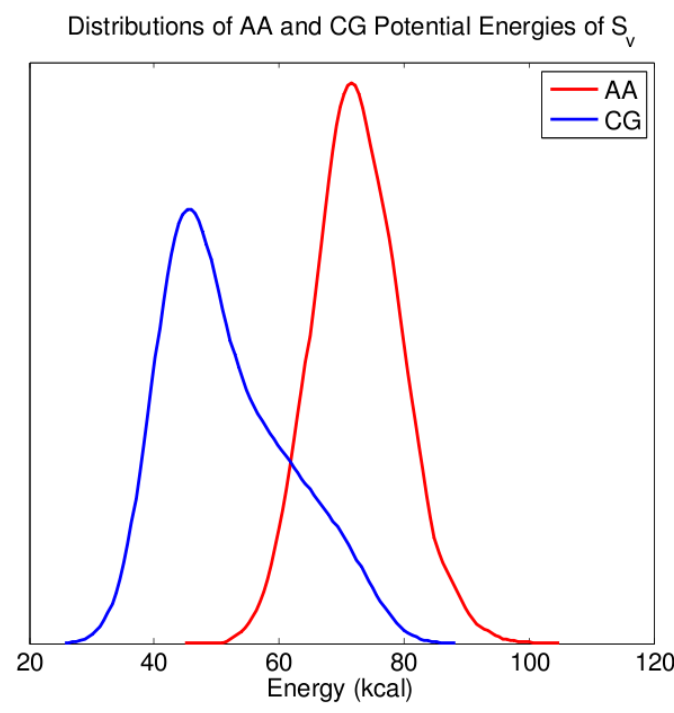

(a)

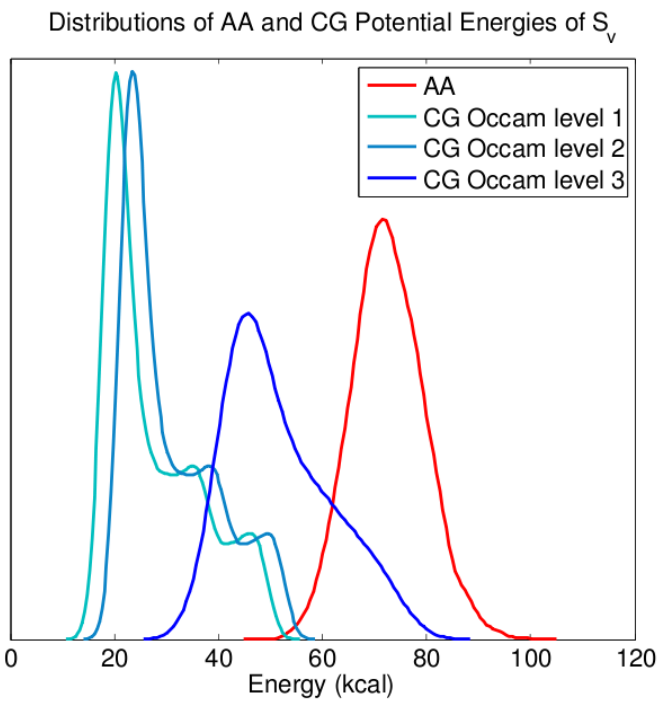

(b)

Figure 10: Comparison of the AA and CG distributions of the validation observable. The CG observables are computed using the validated parameters from the last Occam level (a). In (b), we see that the CG distributions get closer to the AA distribution with each iteration of the Occam-Plausibility Algorithm.

that inherently copes with uncertainties in model parameters, data, and even the selection the model itself.

Each family of CG models is perceived to be the result of a map $G$ from the AA system to the CG configurations, and each image under the map corresponds to a set of parametric classes of models differing in the conformation of potential energy terms and in model parameters. There can be many maps $G\left(=G_{1}, G_{2}, \cdots, G_{k}\right)$, each corresponding to a decision on the structure of the CG model.

In many cases, key observables and quantities of interest to be predicted by CG models are not sensitive to changes in model parameters. The calculation of variance-based model sensitivities provides an a priori indication of which parameters do and do not influence predictions, and those that do not, can, in theory, be eliminated at the onset. A more qualitative indication of parameter sensitivities can also be provided by scatterplots, which can be used in conjunction with sensitivity calculations. We provided examples of cases in which sensitivities computed for both calibration and prediction scenarios produced consistent indications of parameters that could be removed without appreciably influencing predicted observables.

Once a sensitivity analysis is implemented and irrelevant parameters are discarded, one can partition the surviving model classes into a hierarchy we refer to as Occam categories, in deference to Occam's razor, with model simplicity equated 
to the number of parameters in each model class. We, therefore, need to use only models in lowest category containing a valid model: once a valid model class is identified, it is not necessary to consider other models which, by construction, have more than the minimum number of required parameters. We calibrate these models in a calibration scenario, as discussed in Section 3.2. But which models among a calibrated category are best?

The notion of Bayesian posterior model plausibilities can greatly reduce the ambiguity in model selection and can provide a systematic basis for model selection among classes of CG models for given AA data. The most plausible model in a sensitivity class is determined using (18) and (19) for given AA data.

Is the most plausible model in a category valid? To answer this question requires that we construct a validation scenario (or experiment), select a meaningful AA observables and, importantly, select a tolerance that we set as the tolerable accuracy we will accept for the models's ability to predict the observable. We impose conditions such as (23), (25), and (26). If these criteria are met, the model is declared "valid", even though we understand that the choice of the observable, the means to measure prediction accuracy, and the chance of the tolerance $\gamma$ are subjective choices: different choices may lead to the conclusion that the model is invalid (hence, one sometimes claims a model is "not invalid" as a weaker conclusion than the model is "valid").

If the model is invalid, we must move to a higher Occam category of models. If models in all categories prove to be invalid, we must redefine the AA-to-CG map, going to another family of model classes. The choice of a $G_{k}$ more likely to succeed is, at this point, a subjective decision, based largely on prior information and the experience of the modeler.

We refer to the process just described and demonstrated in this work as the Occam-Plausibility Algorithm. Several possibilities for improvement and refinement of steps in the process exist, and will hopefully be the subject of future work.

\section{Acknowledgments}

This material is based upon work supported by the U.S. Department of Energy Office

of Science, Office of Advanced Scientific Computing Research, Applied Mathematics program under Award Number DE-5C0009286. We like to acknowledge the many fruitful discussions with Peter Rossky and Eric Wright, from which we've benefited greatly, and earlier interactions with Serge Prudhomme.

\section{References}

[1] M. Adams, D. Higdon, et al., editors. Assessing the Reliability of Complex Models: Mathematical and Statistical Foundations of Verification, Validation, 
and Uncertainty Quantification. The National Academic Press, Washington, D.C., 2012.

[2] I. Babuška, R. Tempone, and F. Nobile. A systematic approach to model validation based on Bayesian updates and prediction-related rejection criteria. Computer Methods in Applied Mechanics and Engineering, 197:2517-2539, 2008.

[3] P. T. Bauman, J. T. Oden, and S. Prudhomme. Adaptive multiscale modeling of polymeric materials with Arlequin coupling and Goals algorithms. Computational Methods in Applied Mechanics and Engineering, 198:799-818, 2009.

[4] J. L. Beck and K.-V. Yuan. Model selection using response measurements: Bayesian probabilistic approach. Journal of Engineering Mechanics, 130(2):192-203, 2004.

[5] K. Binder. Monte Carlo and Molecular Dynamics Simulations in Polymer Science. Oxford Univ. Press, New York, 1995, 1995.

[6] E. Brini, V. Marcon, and N. F. A. van der Vegt. Conditional reversible work method for molecular coarse graining applications. 13(22):10468-10474, 2011.

[7] E. Brini and N. F. A. van der Vegt. Chemically transferable coarse-grained potentials from conditional reverse work calculations. The Journal of Chemical Physics, 137(15):154113, 2012.

[8] B. R. Brooks, R. E. Bruccoleri, B. D. Olafson, D. J. States, S. Swaminathan, and M. Karplus. CHARMM: A program for macromolecular energy, minimization and dynamics calculations. Journal of Computational Chemistry, 4(2):187$217,1983$.

[9] S. P. Carmichael and M. S. Shell. A new multiscale algorithm and its application to coarse-grained peptide models for self-assembly. The Journal of Physical Chemistry B, 116(29):8383-8393, 2012.

[10] A. Chaimovich and M. S. Shell. Coarse-graining errors and numerical optimization using a relative entropy framework. The Journal of Chemical Physics, 134(9):094112, 2011.

[11] W. D. Cornell, P. Cieplak, C. I. Bayly, I. R. Gould, K. M. Merz, Jr., D. M. Ferguson, D. C. Spellmeyer, T. Fox, J. W. Caldwell, and P. A. Kollman. A second generation force field for the simulation of proteins, nucleic acids, and organic molecules. Journal of the American Chemical Society, 117(19):51795197, 1995. 
[12] R. I. Cukier, C. M. Fortuin, K. E. Shuler, A. G. Petschek, and J. H. Schaibly. Study of the sensitivity of coupled reaction systems to uncertainties in rate coefficients. i theory. The Journal of Chemical Physics, 59(8):3873-3878, 1973.

[13] F. Ercolessi and J. B. Adams. Interatomic potentials from first-principles calculations: The force-matching method. Europhysics Letters, 26(8):583, 1994.

[14] K. Farrell and J. T. Oden. Statistical calibration and validation methods of coarse-grained and macro models of atomic systems. ICES REPORT 12-45, 2012 .

[15] K. Farrell and J. T. Oden. Calibration and validation of coarse-grained models of atomic systems: Application to semiconductor manufacturing. Comput. Mech., 54(1):3-19, July 2014.

[16] P. J. Flory. Thermodynamics of high polymer solutions. The Journal of Computational Physics, 10(1):51-61, 1942.

[17] T. Homma and A. Saltelli. Importance measures in global sensitivity analysis of nonlinear models. Reliability Engineering and System Safety, 52(1):1 - 17, 1996.

[18] D. Hossain, M. Tschopp, D. Ward, J. Bouvard, P. Wang, and M. Horstemeyer. Molecular dynamics simulations of deformation mechanisms of amorphous polyethylene. Polymer, 51(25):6071 - 6083, 2010.

[19] M. L. Huggins. Solutions of long chaim compounds. The Journal of Chemical Physics, 9(5):440, 1941.

[20] S. Izvekov. Towards an understanding of many-particle effects in hydrophobic association in methane solutions. The Journal of Chemical Physics, 134(3):034104, 2011.

[21] S. Izvekov, P. W. Chung, and B. M. Rice. The multiscale coarse-graining method: Assessing its accuracy and introducing density dependent coarsegraining potentials. The Journal of Chemical Physics, 133(6):064109, 2010.

[22] S. Izvekov, M. Parrienllo, C. J. Burnham, and G. A. Voth. Effective force fields for condensed phase systems from ab initio molecular dynamics simulation: A new method for force matching. The Journal of Chemical Physics, 120(23):10896-10913, 2004.

[23] S. Izvekov and G. A. Voth. A multiscale coarse-graining method for biomolecular systems. The Journal of Physical Chemistry B, 109(7):2469-2473, 2005.

[24] S. Izvekov and G. A. Voth. Multiscale coarse graining of liquid-state systems. The Journal of Chemical Physics, 123(13):134105, 2005. 
[25] S. Izvekov and G. A. Voth. Modeling real dynamics in the coarse-grained representation of condensed phase systems. The Journal of Chemical Physics, 125(15):151101, 2006.

[26] E. T. Jaynes. Probability Theory: The Logic of Science. Cambridge University Press, Cambridge, 2003.

[27] W. L. Jorgensen, D. S. Maxwell, and J. Tirado-Rives. Development and testing of the OPLS all-atom force field on conformational energetics and properties of organic liquids. Journal of the American Chemical Society, 118(45):1122511236, 1996.

[28] W. L. Jorgensen and J. Tirado-Rives. The OPLS potential functions for proteins. Energy minimizations for crystals of cyclic peptides and crambin. Journal of the American Chemical Society, 110(6):1657-1666, 1988.

[29] Y. Li, B. C. Abberton, M. Kröger, and W. K. Liu. Challenges in multiscale modeling of polymer dynamics. Polymers, 5(2):751-832, 2013.

[30] A. P. Lyubartsev and A. Laaksonen. Calculation of effective interaction potentials from radial distribution functions: A reverse Monte Carlo approach. Physical Review E, 52(4):3730-3737, 1995.

[31] A. P. Lyubartsev and A. Laaksonen. Effective potentials for ion-DNA interactions. The Journal of Chemical Physics, 111(24):11207, 1999.

[32] R. L. McGreevy and L. Pusztai. Reverse Monte Carlo simulation: A new technique for the determination of disordered structures. Molecular Simulation, 1(6):359-367.

[33] J. W. Mullinax and W. G. Noid. Extended ensemble approach for deriving transferable coarse-grained potentials. The Journal of Chemical Physics, 131(10):104110, 2009.

[34] W. G. Noid. Perspective: Coarse-grained models for biomolecular systems. The Journal of Chemical Physics, 139:090901, 2013.

[35] W. G. Noid, J.-W. Chu, G. S. Ayton, V. Krishna, S. Izvekov, G. A. Voth, A. Das, and H. C. Andersen. The multiscale coarse-graining method. I. A rigorous bridge between atomistic and coarse-grained models. The Journal of Chemical Physics, 128:244114, 2008.

[36] W. G. Noid, P. Liu, Y. Wang, J.-W. Chu, G. S. Ayton, S. Izvekov, H. C. Andersen, and G. A. Voth. The multiscale coarse-graining method. II. Numerical implementation for coarse-grained molecular models. The Journal of Chemical Physics, 128:244115, 2008. 
[37] J. T. Oden, A. Hawkins, and S. Prudhomme. General diffuse-interface theories and an approach to predictive tumor growth modeling. Mathematical Models and Methods of Applied Science, 20(3):1-41, 2010.

[38] J. T. Oden, R. Moser, and O. Ghattas. Computer predictions with quantified uncertainty, Part I. SIAM News, 43(9), November 2010.

[39] J. T. Oden, R. Moser, and O. Ghattas. Computer predictions with quantified uncertainty, Part II. SIAM News, 43(10), December 2010.

[40] J. T. Oden, E. E. Prudencio, and P. T. Bauman. Virtual model validation of complex multiscale systems: Applications to nonlinear elastostatics. Computer Methods in Applied Mechanics and Engineering, 266:162-184.

[41] J. T. Oden, E. E. Prudencio, and A. Hawkins-Daarud. Selection and assessment of phenomenological models of tumor growth. Mathematical Models and Methods in Applied Sciences, 23(7):1309-1338, 2013.

[42] J. T. Padding and W. J. Briels. Uncrossability constraints in mesoscopic polymer melt simulations: Non-rouse behavior of c120h242. The Journal of Chemical Physics, 115(6):2846-2859, 2001.

[43] J. T. Padding and W. J. Briels. Zero-shear stress relaxation and long time dynamics of a linear polyethylene melt: A test of rouse theory. The Journal of Chemical Physics, 114(19):8685-8693, 2001.

[44] S. Plimpton. Fast parallel algorithms for short-range molecular dynamics. Journal of Computational Physics, 117:1-19, 1995.

[45] E. Prudencio and K. Schulz. The parallel c++ statistical library queso: Quantification of uncertainty for estimation, simulation and optimization. In M. Alexander, P. DAmbra, A. Belloum, G. Bosilca, M. Cannataro, M. Danelutto, B. Martino, M. Gerndt, E. Jeannot, R. Namyst, J. Roman, S. Scott, J. Traff, G. Valle, and J. Weidendorfer, editors, Euro-Par 2011: Parallel Processing Workshops, volume 7155 of Lecture Notes in Computer Science, pages 398-407. Springer Berlin Heidelberg, 2012.

[46] D. Reith, M. Pütz, and F. Müller-Plathe. Deriving effective mesoscale potentials from atomistic simulations. The Journal of Computational Chemistry, 24(13):1624-1636, 2003.

[47] A. Saltelli. Making best use of model evaluations to compute sensitivity indices. Computer Physics Communications, 145(2):280 - 297, 2002.

[48] A. Saltelli, P. Annoni, I. Azzini, F. Campolongo, M. Ratto, and S. Tarantola. Variance based sensitivity analysis of model output. design and estimator for 
the total sensitivity index. Computer Physics Communications, 181(2):259 $270,2010$.

[49] A. Saltelli, K. Chan, and E. Scott. Sensitivity Analysis. Number no. 2008 in Wiley paperback series. Wiley, 2009.

[50] A. Saltelli, M. Ratto, T. Andres, F. Campolongo, J. Cariboni, D. Gatelli, M. Saisana, and S. Tarantola. Global Sensitivity Analysis: The Primer. Wiley, 2008.

[51] A. Saltelli and S. Tarantola. On the relative importance of input factors in mathematical models. Journal of the American Statistical Association, 97(459):702-709, 2002.

[52] M. S. Shell. The relative entropy is fundamental to multiscale and inverse thermodynamic problems. The Journal of Chemical Physics, 129(14):144108, 2008 .

[53] Q. Shi, S. Izvekov, and G. A. Voth. Mixed atomistic and coarse-grained molecular dynamics: Simulation of membrance-bound ion channel. The Journal of Physical Chemistry B, 110(31):15045-15048, 2006.

[54] I. M. Sobol'. Sensitivity estimates for nonlinear mathematical models. Matematicheskoe Modelirovanie, 2:112-118, 1990.

[55] I. M. Sobol'. Sensitivity analysis for non-linear mathematical models. Mathematical Modeling and Computational Experiment, 1:407-414, 1993.

[56] P. K. Weiner and P. A. Kollman. AMBER: Assisted Model Building with Energy Refinement. A general program for modeling molecules and their interactions. Journal of Computational Chemistry, 2(3):287-303, 1981.

[57] J. Zhou, I. F. Thorpe, S. Izvekov, and G. A. Voth. Coarse-grained peptide modeling using a systematic multiscale approach. Biophysical Journal, 92(12):42894303, 2007.

\section{A Sensitivity Analysis}

Sensitivity analysis is the study of how the change in input factors of a model, qualitatively and quantitatively, affects the variation and uncertainty in the model outputs. Among the various existing techniques for sensitivity analysis of model output, variance-based methods $[12,54,55]$ has been proven to be effective and well suited for practical application. In these methods the sensitivity of the output to an input variable is measured by the amount of (conditional) variance in the output caused by that input. Variance-based methods are model independent and address interaction effects among input factors. 


\section{A.1 Decomposition of Variance}

Suppose a model $Y=f\left(\theta_{1}, \theta_{2}, \ldots, \theta_{k}\right)$ with $k$ uncertain input factors $\theta_{i}$, where $Y$ is model output and $f$ is a square integrable function over the $k$-dimensional unit hypercube,

$$
\Omega^{k}=\left(\boldsymbol{\theta} \mid 0 \leq \theta_{i} \leq 1 ; i=1, \ldots, k\right) .
$$

Using the Hoeffding decomposition of $f[54,55]$ and conditional expectations of the model, $E\left(Y \mid \theta_{i}\right)$, one can retrieve the following decomposition of the output variance, $V(Y)$,

$$
V(Y)=\sum_{i} V_{i}+\sum_{i} \sum_{j>i} V_{i j}+\cdots+V_{12 \ldots k}
$$

where

$$
\begin{array}{ccc}
V_{i}=V\left(f_{i}\left(\theta_{i}\right)\right)= & V_{\theta_{i}}\left(E_{\boldsymbol{\theta}_{\sim i}}\left(Y \mid \theta_{i}\right)\right) \\
V_{i j}=V\left(f_{i j}\left(\theta_{i}, \theta_{j}\right)\right)= & V_{\theta_{i} \theta_{j}}\left(E_{\boldsymbol{\theta}_{\sim i j}}\left(Y \mid \theta_{i}, \theta_{j}\right)\right) \quad-V_{\theta_{i}}\left(E_{\boldsymbol{\theta}_{\sim i}}\left(Y \mid \theta_{i}\right)\right) \\
& -V_{\theta_{j}}\left(E_{\boldsymbol{\theta}_{\sim j}}\left(Y \mid \theta_{j}\right)\right),
\end{array}
$$

and so on for higher order terms. In (43) $\theta_{i}$ is the $i$-th factor, $\boldsymbol{\theta}_{\sim i}$ denotes the matrix of all factors but $\theta_{i}, V_{\theta_{i}}\left(E_{\boldsymbol{\theta}_{\sim i}}\left(Y \mid \theta_{i}\right)\right)$ and $V_{\theta_{j}}\left(E_{\boldsymbol{\theta}_{\sim j}}\left(Y \mid \theta_{j}\right)\right)$ are the first-order effects, and $V_{\theta_{i} \theta_{j}}\left(E_{\boldsymbol{\theta}_{\sim i j}}\left(Y \mid \theta_{i}, \theta_{j}\right)\right)$ is the joint effect of the pair $\left(\theta_{i} ; \theta_{j}\right)$ on $Y$. In $V_{\theta_{i}}\left(E_{\theta_{\sim i}}\left(Y \mid \theta_{i}\right)\right)$, the inner expectation operator accounts for the mean of $Y$ taken over all possible values of $\boldsymbol{\theta}_{\sim i}$ while keeping $\theta_{i}$ fixed, and the outer variance is taken over all possible values of $\theta_{i}$. Therefore, $V_{\theta_{i}}\left(E_{\boldsymbol{\theta}_{\sim i}}\left(Y \mid \theta_{i}\right)\right)$ is the expected reduction in variance that would be obtained when $\theta_{i}$ is fixed.

\section{A.2 Sensitivity indices}

For non-additive models (e.g. the molecular models under study), the response of $Y$ depends on the interaction between $\theta_{i}$ and $\theta_{j}$. Dividing (42) by $V(Y)$, forms the sensitivity indices $S_{i}, S_{i j}, \cdots, S_{12 \ldots k}$, yielding

$$
\sum_{i} S_{i}+\sum_{i} \sum_{j>i} S_{i j}+\ldots+S_{12 \ldots k}=1 .
$$

Decomposing the variance by conditioning with respect to all factors but $X_{i}$,

$$
V(Y)=V_{\boldsymbol{\theta}_{\sim i}}\left(E_{\theta_{i}}\left(Y \mid \boldsymbol{\theta}_{\sim i}\right)\right)+E_{\boldsymbol{\theta}_{\sim i}}\left(V_{\theta_{i}}\left(Y \mid \boldsymbol{\theta}_{\sim i}\right)\right) .
$$

and dividing both sides by $V(Y)$, results in a variance-based sensitivity measure, the so-called the total effect index $[17,51]$,

$$
S_{T_{i}}=\frac{E_{\boldsymbol{\theta}_{\sim i}}\left(V_{\theta_{i}}\left(Y \mid \boldsymbol{\theta}_{\sim i}\right)\right)}{V(Y)}=1-\frac{V_{\boldsymbol{\theta}_{\sim i}}\left(E_{\theta_{i}}\left(Y \mid \boldsymbol{\theta}_{\sim i}\right)\right)}{V(Y)},
$$


where $E_{\boldsymbol{\theta}_{\sim i}}\left(V_{X_{i}}\left(Y \mid \boldsymbol{\theta}_{\sim i}\right)\right)$ is the remaining variance of $Y$ for fixed values of $\theta_{i}$ and $V_{\boldsymbol{\theta}_{\sim i}}\left(E_{\theta_{i}}\left(Y \mid \boldsymbol{\theta}_{\sim i}\right)\right)$ is the expected reduction in variance if all values other than $\theta_{i}$ are fixed.

Total effect $S_{T_{i}}$ measures the total contribution to the output variation due to factor $\theta_{i}$. Small values of the total effect index $\left(S_{T_{i}} \approx 0\right)$ imply that $X_{i}$ can be fixed at any value within its range of variability (uncertainty) without appreciably affecting the output. $S_{T_{i}}$ provides information on the non-additive feature of model. Note that since the interaction effect among factors $\theta_{i}$ and $\theta_{j}$ is counted in both $S_{T_{i}}$ and $S_{T_{j}}$, the sum of the $S_{T_{i}}$ will be larger than $1, \sum_{i=1}^{k} S_{T_{i}} \geq 1$.

\section{A.3 Computation of the sensitivity indices}

Saltelli $[47,48,50]$ presented a Monte Carlo scheme to compute the indices with a reduced computational cost of evaluating multi-dimensional integrals. The method is the extension of the original approach proposed by Sobol' $[54,55]$ and Homma and Saltelli [17] with the following procedure:

- Generate two $N \times k$ independent sampling matrices, $\mathbf{A}$ and $\mathbf{B}$, where each row is a sample point in the hyperspace of $k$ dimensions. $N$ is called a base sample with respect to the probability distributions of the input variables.

- Define a matrix $\mathbf{D}_{i}$ formed by all columns of $\mathbf{A}$ except the $i$ th column, which is from $\mathbf{B}$.

- Compute the model outputs for all the matrices (i.e. $N \times 1$ vectors of model outputs):

$$
\mathcal{Y}_{A}=f(\mathbf{A}) \quad ; \quad \mathcal{Y}_{B}=f(\mathbf{B}) \quad ; \quad \mathcal{Y}_{D_{i}}=f\left(\mathbf{D}_{i}\right) .
$$

- The total-effect indices can be estimated by,

$$
S_{T_{i}} \approx 1-\frac{\frac{1}{N} \sum_{j=1}^{N} \mathcal{Y}_{A}^{(j)} \mathcal{Y}_{D_{i}}^{(j)}-\left(\frac{1}{N} \sum_{j=1}^{N} \mathcal{Y}_{A}^{(j)}\right)^{2}}{\frac{1}{N} \sum_{j=1}^{N} \mathcal{Y}_{A}^{(j)} \mathcal{Y}_{A}^{(j)}-\left(\frac{1}{N} \sum_{j=1}^{N} \mathcal{Y}_{A}^{(j)}\right)^{2}}
$$

The relation (48) can be obtained by substituting the Monte-Carlo estimators proposed by Saltelli [47],

$$
V_{\boldsymbol{\theta}_{\sim i}}\left[E_{\theta_{i}}\left(Y \mid \boldsymbol{\theta}_{\sim i}\right)\right] \approx \frac{1}{N} \sum_{j=1}^{N} \mathcal{Y}_{A}^{(j)} \mathcal{Y}_{D_{i}}^{(j)}-E^{2}(Y),
$$

along with

$$
V(Y)=E\left(Y^{2}\right)-E^{2}(Y)=\frac{1}{N} \sum_{j=1}^{N} \mathcal{Y}_{A}^{(j)} \mathcal{Y}_{A}^{(j)}-\left(\frac{1}{N} \sum_{j=1}^{N} \mathcal{Y}_{A}^{(j)}\right)^{2}
$$

into (46). 


\title{
A Bayesian Framework for Adaptive Selection, Calibration, and Validation of Coarse-Grained Models of Atomistic Systems
}

\author{
Kathryn Farrell, J. Tinsley Oden, and Danial Faghihi \\ Institute for Computational Engineering and Sciences \\ The University of Texas at Austin \\ kfarrell@ices.utexas.edu, oden@ices.utexas.edu, danial@ices.utexas.edu
}

February 16, 2015

\begin{abstract}
A general adaptive modeling algorithm for selection and validation of coarsegrained models of atomistic systems is presented. A Bayesian framework is developed to address uncertainties in parameters, data, and model selection. Algorithms for computing output sensitivities to parameter variances, model evidence and posterior model plausibilities for given data, and for computing what are referred to as Occam Categories in reference to a rough measure of model simplicity, make up components of the overall approach. Computational results are provided for representative applications.
\end{abstract}

Keywords: Coarse graining models, Bayesian inference, output sensitivities, model plausibility, model validation.

\section{Introduction}

The subject of model validation in the presence of uncertainties - in observational data, in model parameters, and uncertainties in the generally subjective process of selecting the model itself - lies at the very foundations of the scientific method. Scientific knowledge is acquired through observations of physical events and through the development of scientific hypotheses on the causes of the events. The former requires the acquisition of relevant observational data and the latter are the consequences of inductive logic leading to mathematical and computational models, the validity of which must be tested against experiments or observations.

Here we consider issues of model selection and validation in connection with coarse graining of atomic systems: the creation of models of atomistic systems by 
aggregating clusters of atoms into "beads" or "super atoms" so as to dramatically reduce the number of degrees of freedom and also to extend time scales in which physical quantities of interest can be observed. The overriding issues in developing coarse-grained $(\mathrm{CG})$ models are how accurately they approximate key quantities of interest captured by the all-atom (AA) system; that is, is the CG model valid in some sense, and, more fundamentally, how does one select the CG model itself to faithfully represent relevant properties of the AA model? It is important that inherent uncertainties encountered in each step of the model validation and selection process must also be taken into account. In this study, a Bayesian framework is developed to address these issues.

A large literature on CG models exists, going back over a half century as early versions of CG approximations appeared in the 1940's, with more general approaches appearing decades later in computer implementations (e.g. $[22,25])$. The recent survey of Noid [42] of CG models of bio-molecular system cites almost 600 works and the survey of $\mathrm{Li}$ et al. [36] with over 400 references attests to the great interest in CG methodologies. Among methods proposed for constructing CG models using AA-model data, we mention force-matching methods [18, 2831], the so-called "multiscale coarse-graining" methods of Izvekov and Voth et al. $[26,27,31,61,66]$ and Das and Andersen $[16,17]$ which were shown to be "physically consistent" in [41, 43, 44], the iterative Boltzmann inversion methods of [54] in which CG parameters are chosen to fit specific probability distributions in AA systems, the Reverse Monte Carlo (RMC) method [38-40], the Conditional Reverse Work (CRW) method [9, 10], and the methods of Shell et al. based on parameter selections that minimize the relative entropics of CG and AA systems (e.g. [12, 13, 19, 60]).

The present work, which builds on the general framework for model validation and selection advanced in [21] and [48], follows somewhat different arguments than earlier approaches, as it attempts to make specific the processes of model selection and validation in the presence of uncertainties.

Our general approach toward selecting and validating CG models can be described as an adaptive modeling paradigm that employs Bayesian and information theoretics and consists of the following five components:

I Parametric Model Classes: A family of possible parametric model classes with unknown parameters $\boldsymbol{\theta}$ defining a particular CG interatomic potential.

II Parameter Sensitivity Analysis: A parameter sensitivity analysis performed over the entire set of $\mathcal{M}$ models. Here we employ a variance-based sensitivity analysis that computes the sensitivity of a representative output function (here the total potential energy) due to variations in parameters using Monte-Carlo samples of parameter probability densities, retaining only parameters that significantly influence the output.

III Occam Categories: We partition the surviving models into categories numbered according to the number of unknown parameters in each model. Here we equate 
the number of parameters to the simplicity of the model in the spirit of Occam's Razor, knowing that this is not always a precise designation.

IV Model Plausibilities and Parameters Calibration: For models in a given set, we compute the Bayesian posterior plausibility of each model for given AA data in a calibration scenario. The use of model plausibilities in selecting CG models is discussed in $[20,21,49]$. The calibration scenario is viewed as an initial characterization of probability densities of parameters for models of representative molecules that are to be tested in one or more validation tests. Upon calculating plausibilities, the most plausible is selected.

V Validation Tests: One or more validation tests are designed to test the accuracy with which the most plausible model from IV predicts CG observables. A validation criterion must be specified to judge if the prediction is adequate and that, therefore, the model is not invalid ("valid"). If the model is valid, the process is terminated. The validated model is then used to solve the forward problem for the target quantities of interest (QoI) and the uncertainty in the QoI is quantified using standard measures.

Other proposals for Bayesian calibration methods for molecular systems can be found in the papers of Liu, et al. [37], Espanñol and Zúñiga [19], and Wang et al. [64], and more recently in the work of Angelikopoulos et al. [2, 3]. Also relevant are the works of Das and Anderson [16, 17] on their multiscale coarse-graining methods.

Following this introduction, we present an overview modeling atomistic systems and creating a coarse-grained model. In Section 3, a Bayesian framework for building predictive models is detailed. Section 4 presents the Occam-Plausibility Algorithm, which pairs the concept of Occam's Razor with the Bayesian framework laid down in Section 3. An example application of this algorithm to coarse-grained polyethylene is given in Section 5. Concluding remarks can be found in Section 6 .

\section{The Problem Setting}

\subsection{The AA Model}

We begin with the general process of creating CG models designed to deliver approximations of properties of a "ground-truth" all-atom (AA) model, the properties of which are assumed to be known (the force field, parameters, etc.). The AA model thus provides synthetic observational data for calibrating and validating the $\mathrm{CG}$ model for specific observables and quantities of interest (QoI). For specificity, the behavior of the atomic system is assumed to be captured through molecular dynamics (MD) simulations, implemented using a hardened and generally accepted MD code. Also for simplicity, and without loss in generality, we restrict attentions to configurational energies of canonical ensembles of AA systems. In the present 
investigation, we employ LAMMPS [52]. A force field (the functional form of the potential energy) of the general form calibrated in $[34,35]$ is employed,

$$
u(\mathbf{r})=V_{\text {bond }}(\mathbf{r})+V_{\text {angle }}(\mathbf{r})+V_{\text {dihedral }}(\mathbf{r})+V_{\text {non-bonded }}(\mathbf{r})+V_{\text {coulomb }}(\mathbf{r}),
$$

where $\mathbf{r}=\mathbf{r}^{n}=\left\{\mathbf{r}_{1}, \mathbf{r}_{2}, \ldots, \mathbf{r}_{n}\right\}$ is the vector of atomic coordinates in an $n$-atom system, and, as typical examples of energy characterizations,

$$
\begin{aligned}
V_{\text {bond }}(\mathbf{r}) & =\sum_{i=1}^{N_{b}} \frac{1}{2} k_{r i}\left(r_{i}-r_{0 i}\right)^{2}, \\
V_{\text {angle }}(\mathbf{r}) & =\sum_{i=1}^{N_{a}} \frac{1}{2} k_{\theta i}\left(\theta_{i}-\theta_{0 i}\right)^{2}, \\
V_{\text {dihedral }}(\mathbf{r}) & =\sum_{i=1}^{N_{d}} \sum_{n=1}^{4} \frac{V_{n} i}{2}\left[1+(-1)^{n-1} \cos \left(n \phi_{i}\right)\right],
\end{aligned}
$$

and, typically, the non-bonded energy is of a Lennard-Jones form,

$$
\begin{aligned}
V_{\text {non-bonded }}(\mathbf{r}) & =\sum_{i=1}^{N_{n b}} \sum_{j>i} 4 \epsilon_{i j}\left(\left(\frac{\sigma_{i j}}{r_{i j}}\right)^{\alpha}-\left(\frac{\sigma_{i j}}{r_{i j}}\right)^{\beta}\right), \quad r_{i j} \leq r_{c}, \\
V_{\text {columb }}(\mathbf{r}) & =\sum_{i=1}^{N_{q}-1} \sum_{j>i}^{N_{q}} 4 \epsilon_{0} \frac{q_{i} q_{j}}{r_{i j}} .
\end{aligned}
$$

Here $N_{b}, N_{a}, \ldots, N_{q}$ are the numbers of representative interaction types, $(\alpha, \beta)$ is the Lennard-Jones (LJ) pair (typically $\alpha=12, \beta=6$ ), $r_{c}$ is the cut-off distance for non-bonded interactions, $\varepsilon_{i j}=\sqrt{\varepsilon_{i i} \varepsilon_{j j}}, \sigma_{i j}=\sqrt{\sigma_{i i} \sigma_{j j}}, \epsilon_{0}$ is the universal permitivity constant, and $r_{i j}=\left\|\mathbf{r}_{i}-\mathbf{r}_{j}\right\|$. Force field constants $k_{r i}, k_{\theta i}, \varepsilon_{i i}$, and $V_{n i}$; geometric constants $r_{0 i}, \theta_{0 i}$, and $\sigma_{i i}$; and the Coulomb charges at atoms $i$ and $j, q_{i}$ and $q_{j}$, respectively, are the force field parameters. Many alternative forms of the potential $u(\mathbf{r})$ can be found in the literature and are used in other well-established MD codes (e.g. $[11,14,65])$. Our general approach can accommodate any of the common potentials used in MD calibrations. Generally, we consider charge-neutral systems so that $V_{\text {coulomb }}=0$.

To provide further focus on modeling issues, we generally confine ourselves to the setting of canonical ensembles, also called NVT ensembles, in which the number of atoms, volume, and temperature are fixed. For such ensembles, the probability with which configurations of the system are accessed is given by the Boltzmann distribution,

$$
\rho(\omega)=\exp \{-\beta u(\omega)+a \beta\}
$$

where $\omega=\mathbf{r}^{n}, \beta=k_{B} T$, $\left(k_{B}\right.$ being the Boltzmann constant, $T$ the absolute temperature, and $a$ a normalization constant). The observables of the system are often 
taken to be ensemble averages of phase functions $q(\omega)$, denoted

$$
\langle q\rangle=\int_{\Gamma_{A A}} \rho(\omega) q(\omega) d \omega,
$$

$\Gamma_{A A}$ being the AA phase-space. However, other quantities may be of interest, depending on the system and application. These may be defined at the modeler's discretion. Note that observables may also be distributions $\pi(q(\omega))$. In general, we invoke the ergodic hypothesis and evaluate (4) for given $q(\omega)$ using samples drawn from MD calculations with, for example, the Nosé-Hoover thermostat employed to maintain constant temperature.

\subsection{The CG Models}

It has been understood for decades that the enormous size and complexity of AA models needed to capture events at length and time scales relevant in countless applications in material science, nanomanufacturing, biomedicine, and other areas, far exceeds the capacity of today's computing systems or even those envisioned many years into the future. Thus, methods for reducing the number of degrees of freedom and lengthening simulation time periods by aggregating atoms into coarse-grained molecular models is viewed as a necessary approach to studies of most atomistic simulations.

Let $\mathbf{R}^{N}=\left\{\mathbf{R}_{1}, \mathbf{R}_{2}, \ldots, \mathbf{R}_{N}\right\}$ denote the molecule ("bead") coordinates in a $\mathrm{CG}$ representation of an AA system which contains $n$ atoms. It is convenient to regard each configuration $\mathbf{R}^{N}$ as the image of a set of atom coordinates $\mathbf{r}^{n} \in$ $\Gamma_{A A}$ under a map $G$, and for simplicity in notation, we write $\mathbf{r}^{n}=\omega$ and $\mathbf{R}^{n}=$ $G(\omega)$. We elaborate on the structure and definition of $G$ in the next section. As we are considering only conformational energies, the resulting Hamiltonian of the CG system is,

$$
H\left(\mathbf{R}^{N} ; \boldsymbol{\theta}\right)=H(G(\omega) ; \boldsymbol{\theta})=U(G(\omega) ; \boldsymbol{\theta})
$$

where $U(.,$.$) is the potential energy and \boldsymbol{\theta}$ is the vector of parameters for the particular CG representation. The general form of $U(.,$.$) is identical to (1) with \mathbf{r}$ replaced by the $N$ CG coordinates $\mathbf{R}^{N}$, and,

$$
\boldsymbol{\theta}=\left\{K_{R i}, R_{0 i}, K_{\theta i}, \theta_{0 i}, V_{n i}, \epsilon_{i i}, \sigma_{i i}\right\},
$$

are the unknown parameters of the CG model. Our goal is to calculate an acceptable approximation of the QoI (4) using the CG model,

$$
Q(\boldsymbol{\theta})=\langle q(\boldsymbol{\theta})\rangle=\int_{\Gamma_{C G}} \rho_{C G}(G(\omega) ; \boldsymbol{\theta}) q(G(\omega)) d G(\omega),
$$

$\Gamma_{C G}$ being the reduced phase space in the $\mathrm{CG}$ model and $\rho_{C G}$ is the CG Boltzmann distribution,

$$
\rho_{C G}=\exp \{-\beta U(G(\omega) ; \boldsymbol{\theta})+\beta A(\boldsymbol{\theta})\}
$$


Thus, we again consider a canonical ensemble with $\mathrm{N}, \mathrm{V}, \mathrm{T}$ fixed. Note that since $\boldsymbol{\theta}$ will be a random variable, stochastically determined as discussed in Section $3, Q(\boldsymbol{\theta})$ is actually a probably density function. Further details are given in Section 3.5.

The significant problems with these approximations are obvious: 1) the number $N$ of $\mathrm{CG}$ particles is not precisely specified (i.e. the choice of the map $G$ is not well-defined); 2) once specified, the choice and structure of the interaction potentials $V_{\text {bond }}, V_{\text {angle }}, \ldots, V_{\text {non-bonded }}$ of (2) is unspecified; 3 ) importantly, the parameters $\boldsymbol{\theta}$ are unknown for every choice of $N$ and every set of potentials chosen; and 4) each step is met with uncertainties in the AA data, the parameters, and the model output.

\subsection{Parametric Model Classes}

The general setting is illustrated in Figure 1, where several possible CG approximations are declared, each with a dependent AA-to-CG map $G$, and for each choice, different choices of intermolecular force fields $V$, such as those in (1) are possible. For each choice of $G$ (equivalently $N$ ) and potentials $V$, the model parameters $\boldsymbol{\theta}$ are unknown.

The idea of mapping a set of atoms in the AA system into a CG bead is standard in literature on coarse-graining methods, but the actual definition of such maps can involve technicalities. For example, " $G: \mathrm{AA} \rightarrow \mathrm{CG}$ " may be a multi-valued relation (not a function) on coordinate pairs $\left(\mathbf{r}_{n}, \mathbf{R}_{N}\right)$ in which an AA coordinate vector $\mathbf{r}_{\alpha}, 1 \leq \alpha \leq n$, is identified with a CG coordinate vector $\mathbf{R}_{A}, 1 \leq A \leq N$, the coordinate labels $\alpha$ being members of an index set $\mathcal{J}_{A}$. In the case of bonded CG systems, for example, an AA coordinate vector can be associated with more than one index set. The CG coordinate vector $\mathbf{R}_{A}$ may be located at any convenient point within the bead " $A$ ", but often is chosen as the mass center $\left(\mathbf{R}_{A}=\sum_{\alpha \in \mathcal{J}_{A}} m_{\alpha} \mathbf{r}_{\alpha} / \sum_{\alpha \in \mathcal{J}_{A}} m_{\alpha}\right.$, it being understood that fractions of atomic masses are distributed to beads sharing atoms). The relationship between AA and CG coordinate vector labels can be expressed as simply $\mathbf{R}_{A}=G_{A}^{\cdot \alpha} \mathbf{r}_{\alpha}$, sum on $\alpha$, where $G_{A}^{\cdot \alpha}$ is the Boolean array, $G_{A}^{\cdot \alpha}=1$ if $\alpha \in \mathcal{J}_{A}$ and $G_{A}^{\cdot \alpha}=0$ if otherwise. We generally write symbolically, $\mathbf{R}^{N}=G\left(\mathbf{r}^{n}\right)=G(\omega),\left(\omega=\mathbf{r}^{n}\right)$.

The CG process can thus lead to a large family $\mathcal{M}$ of possible model classes $\mathcal{M}_{i}$ each with a different AA-to-CG map $G$,

$$
\begin{aligned}
\mathcal{M} & =\left\{\mathcal{M}_{1}, \mathcal{M}_{2}, \ldots, \mathcal{M}_{k}\right\} \\
\mathcal{M}_{i} & =\left\{\mathcal{P}_{i 1}\left(\boldsymbol{\theta}_{i 1}\right), \mathcal{P}_{i 2}\left(\boldsymbol{\theta}_{i 2}\right), \ldots, \mathcal{P}_{i m}\left(\boldsymbol{\theta}_{i m}\right)\right\}, \quad i=1,2, \ldots, k
\end{aligned}
$$

For simplicity in notation, we pick one class $\mathcal{M}\left(=\mathcal{M}_{1}\right)$ and denote its members $\left\{\mathcal{P}_{1}\left(\boldsymbol{\theta}_{1}\right), \mathcal{P}_{2}\left(\boldsymbol{\theta}_{2}\right), \ldots, \mathcal{P}_{m}\left(\boldsymbol{\theta}_{m}\right)\right\}$ 


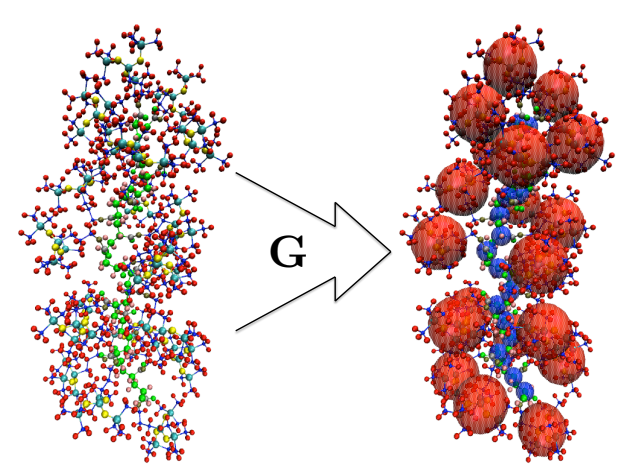

(a)

$$
\mathcal{M}_{k}
$$

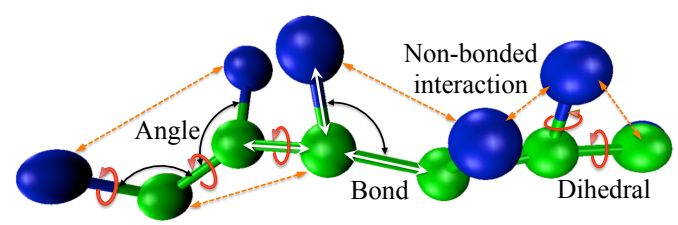

$\mathcal{P}_{k 1}$

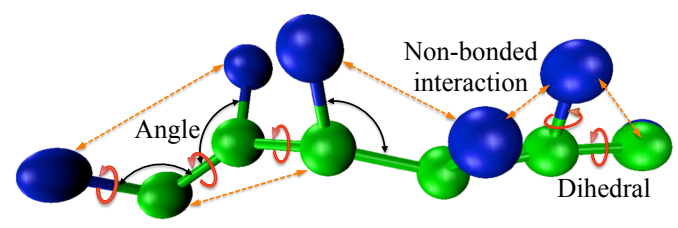

$\mathcal{P}_{k 3}$

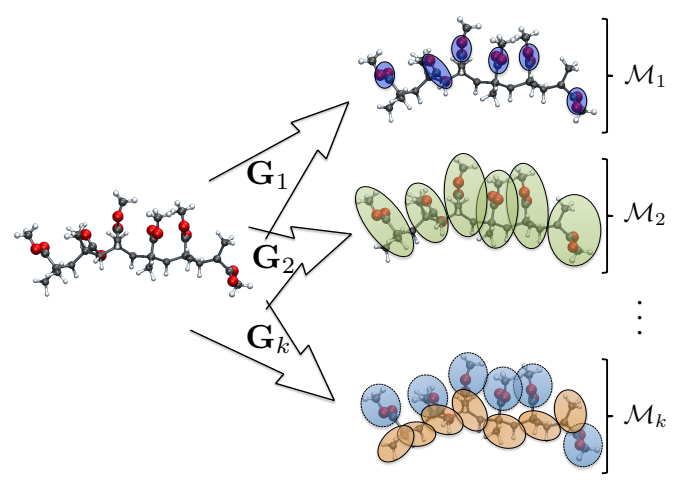

(b)

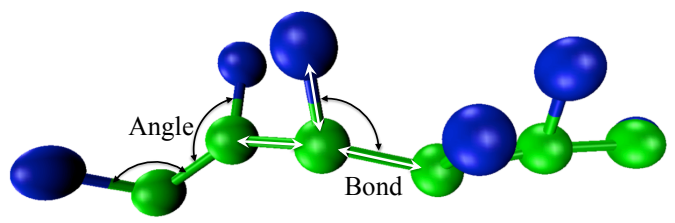

$\mathcal{P}_{k 2}$

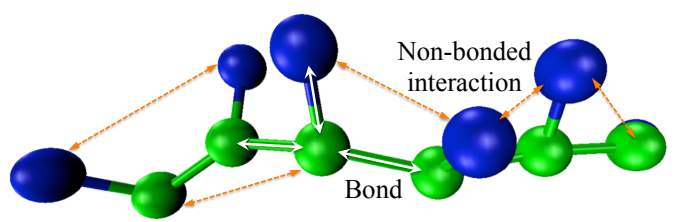

$\mathcal{P}_{k m}$

(c)

Figure 1: (a) Coarse-graining of an all-atom system; (b) different choices of AAto-CG map $G$; (c) different choices of intermolecular force fields $V$, for each map $G$.

\section{Bayesian Framework for Model Validation Under Un- certainty}

To provide an appropriate context for developments in Sections 4 and 5, we outline a Bayesian framework for validation (see, e.g. $[4,20,21,46-48]$ ). Our goal is the following: given AA data in one or more calibration scenarios (defined below) find the most plausible model $\mathcal{P}_{j}\left(\boldsymbol{\theta}_{j}\right) \in \mathcal{M}$, in a parametric model class $\mathcal{M}_{i} \in \mathcal{M}$, and (simultaneously) determine probability density functions (pdfs) representing the uncertainty in parameters of the most plausibe model in the class. Then, having statistically calibrated a model deemed most plausible in $\mathcal{M}$, perform validation tests 


\section{BAYESIAN FRAMEWORK FOR MODEL VALIDATION UNDER}

in validation scenarios to determine if the accuracy with which the model predicts validation observable is sufficient to claim the model is "valid" (not invalid) for the purpose for which it was intended, it being assumed that the validation observables depict in some reasonable way the quantity of interest.

We note that a fundamental tenant in the art and science of model validation is that the notion of validity of a model is only meaningful in connection with specific quantities of interest; a model may be deemed valid for predicting one QoI but invalid for another [48]. Philosophically, the QoI is not an observable; it is data that must be extrapolated beyond the range of data accessible in validation tests. Thus, a challenge in designing validation tests is to choose subjectively observables that represent the QoI. These concepts are illustrated in example calculations to follow.

\subsection{Scenarios}

The idea of calibration, validation, and prediction scenarios is described in several papers $[4,20,21,46-48]$ and is a useful concept for orchestrating the process of model validation. Scenarios are basically restricted solution domains of mathematical models of physical events, the calibration scenarios generally representing those appearing in simple component tests designed to calibrate parameters, while the validation scenarios correspond to more complex subsystem experiments designed to test the accuracy of model prediction against carefully selected observables that hopefully depict key quantities of interest that reside as extrapolations of the observable data. The prediction scenario corresponds to the full forward problem in which a validated model is used to predict quantities of interest.

In general, the calibration, validation, and prediction scenarios are conceptualized as levels in a "prediction pyramid" (see, e.g. $[1,4,20,21,46-48]$ ). The calibration scenario $S_{c}$ is represented by the base of the pyramid where the model accesses calibration data $\mathbf{D}_{c}$. Traveling upwards, the validation scenario $S_{v}$ accesses validation observables represented by validation data $\mathbf{D}_{v}$, and $S_{p}$, the full-system prediction scenario, is at the peak of the pyramid where the non-observable QoI resides.

Computational cost increases as we move up the pyramid. It must be kept in mind that the purpose of developing reduced-order models in the first place is that the enormous high-fidelity or experimental system is, in general, hopelessly impossible to solve or test. The same can be said of the validation scenario models, representing, by definition, as depicting as much as affordable the full prediction scenario. Computing model plausibilities of a set of models of the validation experiment is generally infeasible. The validation test for reasonable atomic and molecular configurations can run for days or weeks on multi-processor machines. The design of appropriate validation experiments is itself a very non-trivial task as they cannot represent a simple perturbation of a calibration test as they must be able to deliver an acceptable information gain (in the posterior) over the calibration experiments. Equally important, the parameter sensitivities of outputs in the validation scenario 
must be essentially the same or close to those of the full system prediction scenario (including the QoI), else the validation experiment is poorly designed and cannot inform the full-system parametric model.

\subsection{Output Sensitivity}

In many cases, one or more model parameters may be ignored or eliminated in parametric models if key model outputs are not affected by changes in the parameter values. One approach, which we adopt in this work, is to employ variance-based methods in which the sensitivity pattern of a model is described by decomposing the variance $V(Y)$ of an output function $Y=Y\left(\theta_{1}, \theta_{2}, \cdots, \theta_{k}\right)$ into contributions from each of the parameters [15, 62, 63]. By using Monte Carlo samples of each parameter, total sensitivity indices are estimated via,

$$
S_{T_{i}}=\frac{E_{\boldsymbol{\theta}_{\sim i}}\left(V_{X_{i}}\left(Y \mid \boldsymbol{\theta}_{\sim i}\right)\right)}{V(Y)}
$$

Here, $\boldsymbol{\theta}_{\sim i}$ is the input vector excluding the $i$ th component $\theta_{i}, V_{X_{i}}\left(Y \mid \boldsymbol{\theta}_{\sim i}\right)$ is the variance due to $\theta_{i}$ of the output produced by $\boldsymbol{\theta}_{\sim i}$ and $E_{\boldsymbol{\theta}_{\sim i}}$ is the conditional expectation of the model given $\boldsymbol{\theta}_{\sim i}$. The indices $S_{T_{i}}$ provide a measure of the sensitivity of each input factor on the total output variance. Further details are given in Appendix A.

A more qualitative route to computing sensitivities is to construct scatterplots [57]. In this method, the parameters are randomly sampled and the output function $Y$ is computed as mentioned above. For each parameter, samples are compared directly to the value of $Y$. A parameter is considered to be important if a clear correlation between the parameter's value and the output value can be seen in the scatterplot patterns. Two examples are discussed in the next subsection.

We assert that when applied to the Bayesian framework discussed in this section, the sensitivity analysis should be performed in both the calibration and prediction scenarios. When implemented in the prediction scenario, the output function should be the QoI. The parameters shown to be important are those that have the most influence on the QoI and should therefore be included in the model calibration. When implemented in the calibration scenario, the output function should be the same observable used as calibration data in the Bayesian update (14). Then, not only are the important parameters influential to the output, we can say that the observable will inform the parameters during calibration. Both points are critical: the parameters should influence the QoI and they need to be informed (updated) by the calibration observable.

\subsubsection{Example: Polyethylene Chain}

In order to reduce the parameter space dimension, quantitative sensitivity analysis is conducted on the CG system to determine the relative level of importance of each parameter to the others. As the first sensitivity scenario, a single chain with 
12 beads, with two carbon atoms per bead, is considered in a constant volume and temperature (i.e. $T=100 \mathrm{~K}$ ) canonical ensemble. The chain is deformed by applying a force to the bead at one end while the other end is constrained. As model output $Y$, we take the difference between average potential energies before and after deformation. That is,

$$
Y(\boldsymbol{\theta})=\Delta\langle U\rangle=\left\langle U\left(\boldsymbol{\theta}, G_{1}\right)\right\rangle-\left\langle U\left(\boldsymbol{\theta}, G_{0}\right)\right\rangle,
$$

where $G_{0}$ is the initial conformation of the chain and $G_{1}$ is the conformation after deformation.

With respect to this observable, the scatterplots for some of the parameters are shown in Fig. 2. These plots were obtained by sampling the parameters from given distributions and computing the model output at each sample point. The distributions assumed are

$$
\begin{array}{rlrl}
r_{0} & \sim \mathcal{U}(0.1,5) ; & & K_{r} \sim \mathcal{U}(0.1,90) ; \\
\theta_{0} \sim \mathcal{U}(80,180) ; & K_{\theta} \sim \mathcal{U}(0,15) ; \\
V_{1} \sim \mathcal{U}(-1,1) ; & V_{2} \sim \mathcal{U}(-1,1) ; \\
V_{2} \sim \mathcal{U}(-1,1) ; & V_{4} \sim \mathcal{U}(-1,1) ; \\
\sigma \sim \mathcal{U}(0.05,7) ; & & \epsilon \sim \mathcal{U}(0.01,5) .
\end{array}
$$

Total sensitivity indices (10) are also estimated using the method described in Section A.3. The result for each parameter is shown in Fig 3. It can be seen qualitatively from scatterplots of Fig. 2 and quantitatively from total sensitivity indices of Fig. 3 that the dihedral parameters, $V_{n}, n=1, \ldots, 4$, do not have a significant effect on the value of the change in potential energy as the polyethylene chain is deformed.

To confirm this result, we compute the same output function $Y=\Delta\langle U\rangle$, now considering three sets of parameters, one in which all the interactions are used in the calculation and the others in which the dihedral and angel parameters are excluded from sampling. The kernel density estimate (KDE) for each case is presented in Fig. 4. The minimal difference between the KDEs due to excluding dihedrals (comparison with the results of excluding angles) indicates the insignificant effect of the dihedral parameters on the change in the average potential energy as the chain is deformed. Therefore, we conclude that these parameters may be excluded from the calibration process.

\subsubsection{Example: Polyethylene System}

A natural question that arises is whether sensitivity tests performed at the calibration scenario remain relevant for the full system or for validation scenarios. To explore this issue, we consider a more complex polyethylene system closer to a prediction scenario consisting of a complex system of polymer chains shown in Fig. 5. A configuration of a polyethylene system initially contained in a volume of 


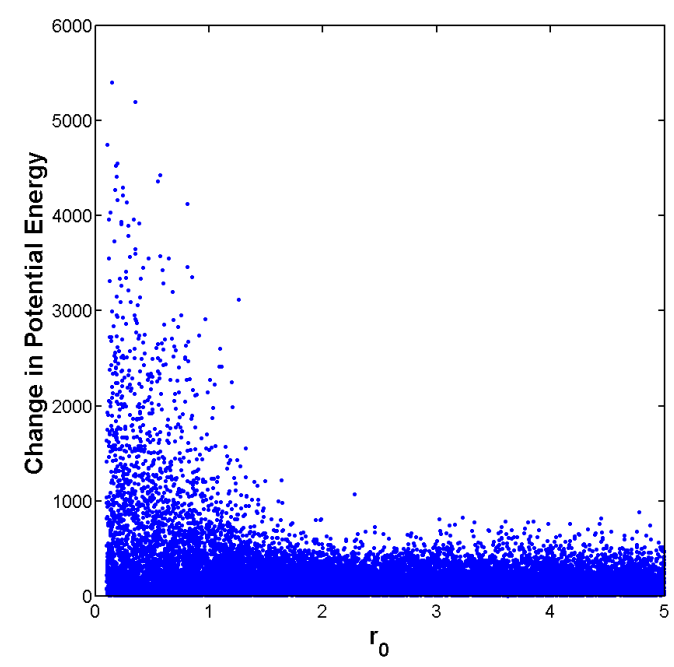

(a)

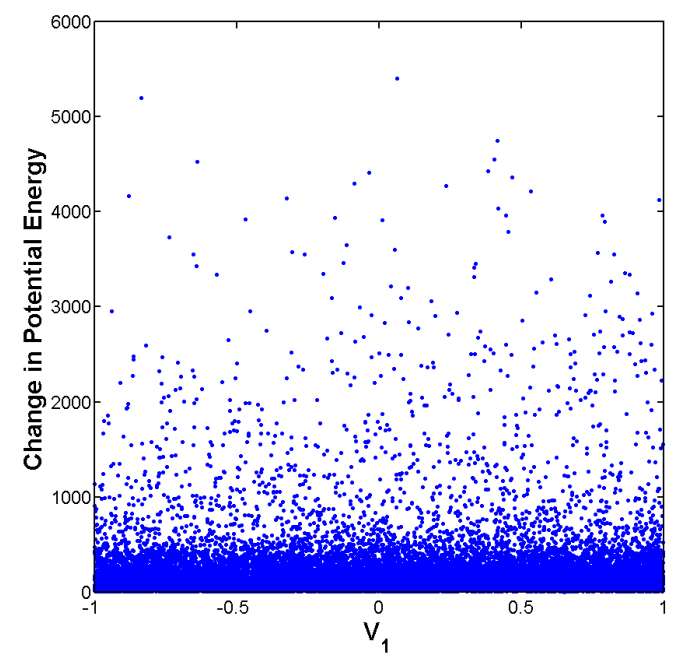

(c)

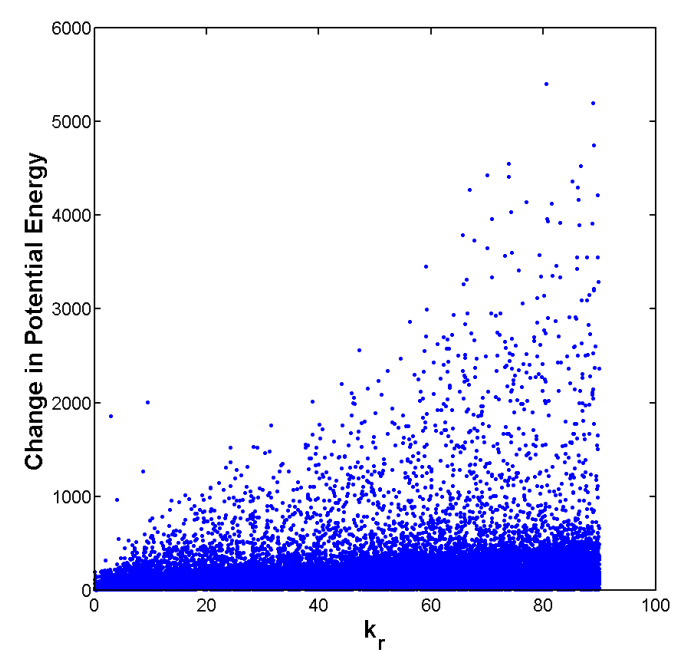

(b)

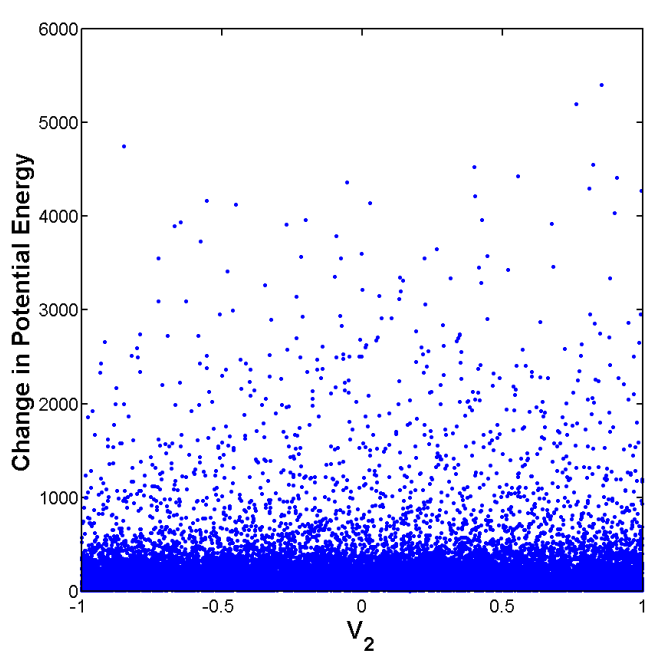

(d)

Figure 2: Scatterplots illustrating a qualitative sensitivity analysis for the polyethylene chain using 40,000 Monte Carlo samples of the parameter space. The observable is the change in potential energy as the chain is deformed.

size $10 \times 10 \times 10 \mathrm{~nm}$ and under biaxial deformation (along the $x$ and $y$ directions) is considered. The initial chain structure is created using a method similar to those previously developed using Monte Carlo self-avoiding random walks [8]. The initial structure shown in Fig. 5 contains 500 chains with 10 CG beads per chain. 


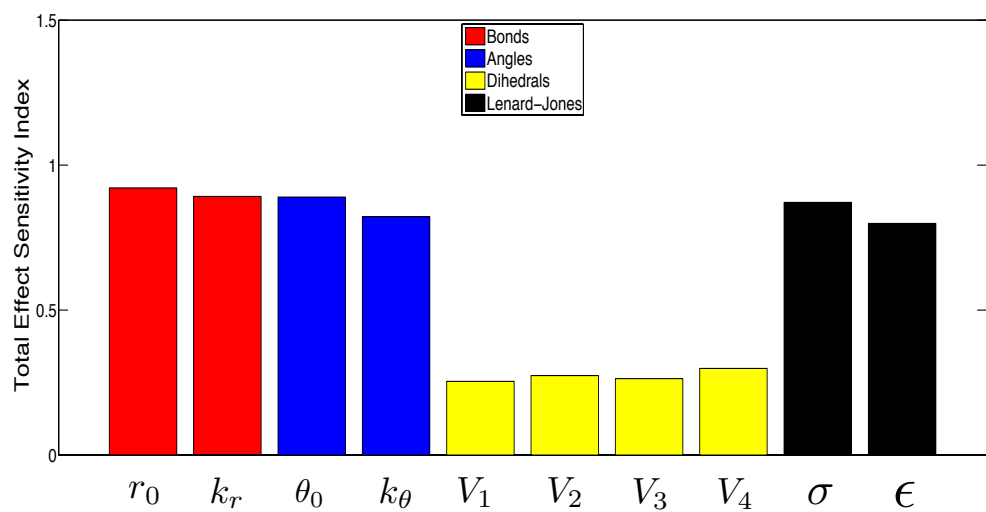

\begin{tabular}{|c|c|}
\hline Parameters & $S_{T}$ \\
\hline \hline$r_{0}$ & 0.92 \\
\hline$k_{r}$ & 0.89 \\
\hline$\theta_{0}$ & 0.89 \\
\hline$k_{\theta}$ & 0.82 \\
\hline$V_{1}$ & 0.25 \\
\hline$V_{2}$ & 0.27 \\
\hline$V_{3}$ & 0.26 \\
\hline$V_{4}$ & 0.30 \\
\hline$\sigma$ & 0.87 \\
\hline$\varepsilon$ & 0.80 \\
\hline
\end{tabular}

Figure 3: Bar chart illustrating the results of the sensitivity analysis using total sensitivity indices for the deformation of the polyethylene chain using 20,000 Monte Carlo samples of the parameter space.

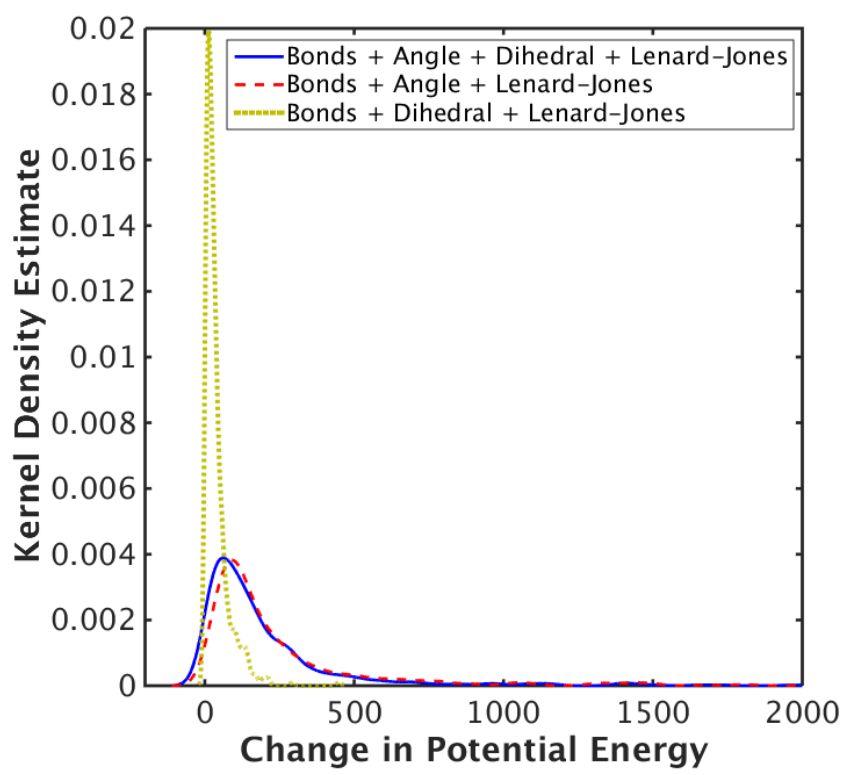

Figure 4: Comparison between the kernel density estimates of the change in potential energy resulting from the deformation of a polyethylene chain for the cases when dihedral and angle parameters are excluded from calculations. Results show 500 Monte Carlo samples of the parameter space.

Before applying the deformation, an equilibration sequence is performed on the initial structure, following the steps presented [24]. The equlibrated polyethylene system is then deformed under biaxial tensile strain applied at a constant strain rate. The mechanical response of the system can be characterized by the variation 
of the Second Piola-Kirchhoff stress against the Lagrangian Green strain in each direction and, correspondingly, against the Helmholtz free energy, here equivalent to the strain-energy density function $W$. As model output $Y$, we take the values of $W$ at $\lambda_{x}=1.3$, where $\lambda_{x}$ (i.e. the principal stretch) is defined as the ratio of the length of the deformed simulation box along the $x$-axis to the undeformed length measured at the end of the equilibration step. That is,

$$
Y(\boldsymbol{\theta})=W\left(\lambda_{x}=1.3\right) .
$$

Scatterplots generated for bond length $r_{0}$ and angle $\theta_{0}$ as well as two dihedral coefficients, $V_{3}$ and $V_{4}$, are shown in Fig. 6. The estimated total effect sensitivity indices for all parameters are presented in Fig. 7. Comparing these results to those in Section 3.2.1, we conclude that the effect of bond length and angle are more pronounced in the larger scale polyethylene system, while the dihedral interactions between the beads remain weaker. The latter observation confirms the assumptions made in $[50,51]$ for simulating large scale amorphous polyethylene and illustrates that, indeed, dihedrals can be excluded from the calibration.

\subsection{Calibration}

Let $\mathbf{D}_{c}$ be the vector of observational data supplied by the AA model for a calibration scenario $S_{c}, \mathbf{D}_{c}$ being a vector in a space $\mathcal{D}$ of observational data. If $d: \Theta \rightarrow \mathcal{D}$ is the parameter-to-observation map provided by model $\mathcal{P}$, and if the discrepancy $\varepsilon$ between observational data and model prediction is additive and represented by a random variable with density $p$, then the density

$$
\pi\left(\mathbf{D}_{c} \mid \boldsymbol{\theta}\right)=p\left(\mathbf{D}_{c}-\mathbf{d}(\boldsymbol{\theta})\right)
$$

is the likelihood function, the conditional probability density of the data given parameters $\boldsymbol{\theta}$. The error $\varepsilon$ represents the sum of the observational noise and error due to model inadequacy $[20,48]$. The density $p$ is often assumed to be Gaussian with covariance matrix defined by parameters that are added to the list of model parameter $\boldsymbol{\theta}$ and are determined by the calibration tests.

The posterior probability density is given by Bayes's rule,

$$
\pi\left(\boldsymbol{\theta} \mid \mathbf{D}_{c}\right)=\frac{\pi\left(\mathbf{D}_{c} \mid \boldsymbol{\theta}\right) \pi(\boldsymbol{\theta})}{\pi\left(\mathbf{D}_{c}\right)}
$$

where $\pi(\boldsymbol{\theta})$ is the prior, representing prior information on $\boldsymbol{\theta}$ and $\pi\left(\mathbf{D}_{c}\right)$ is the evidence,

$$
\pi\left(\mathbf{D}_{c}\right)=\int_{\Theta} \pi\left(\mathbf{D}_{c} \mid \boldsymbol{\theta}\right) \pi(\boldsymbol{\theta}) d \boldsymbol{\theta}
$$

$\Theta$ being the parameter space for model $\mathcal{P}$.

When prior information about parameters can be inferred from data from the AA model, the prior can be computed using the maximum entropy method 
advocated by Jaynes [32, 33]. Using the maximum entropy derivation for prior distributions incorporates any prior information (e.g. from the AA data) we may have about a parameter, e.g. mean, variance, range, etc., as constraints while acknowledging the fact that this information is incomplete. For example, if the mean of a parameter is known, the maximum entropy prior is an exponential distribution. If, in addition, the variance is known, a Gaussian distribution maximizes the entropy. In [21] it is demonstrated that useful prior probabilities can be derived from AA data. Given suitable priors and the likelihood function, the actual posterior distribution (14) is determined by implementing any one of several robust sampling algorithms. In the examples described later, we employ a MCMC (Markov Chain Monte Carlo) algorithm available in the software package QUESO [53].

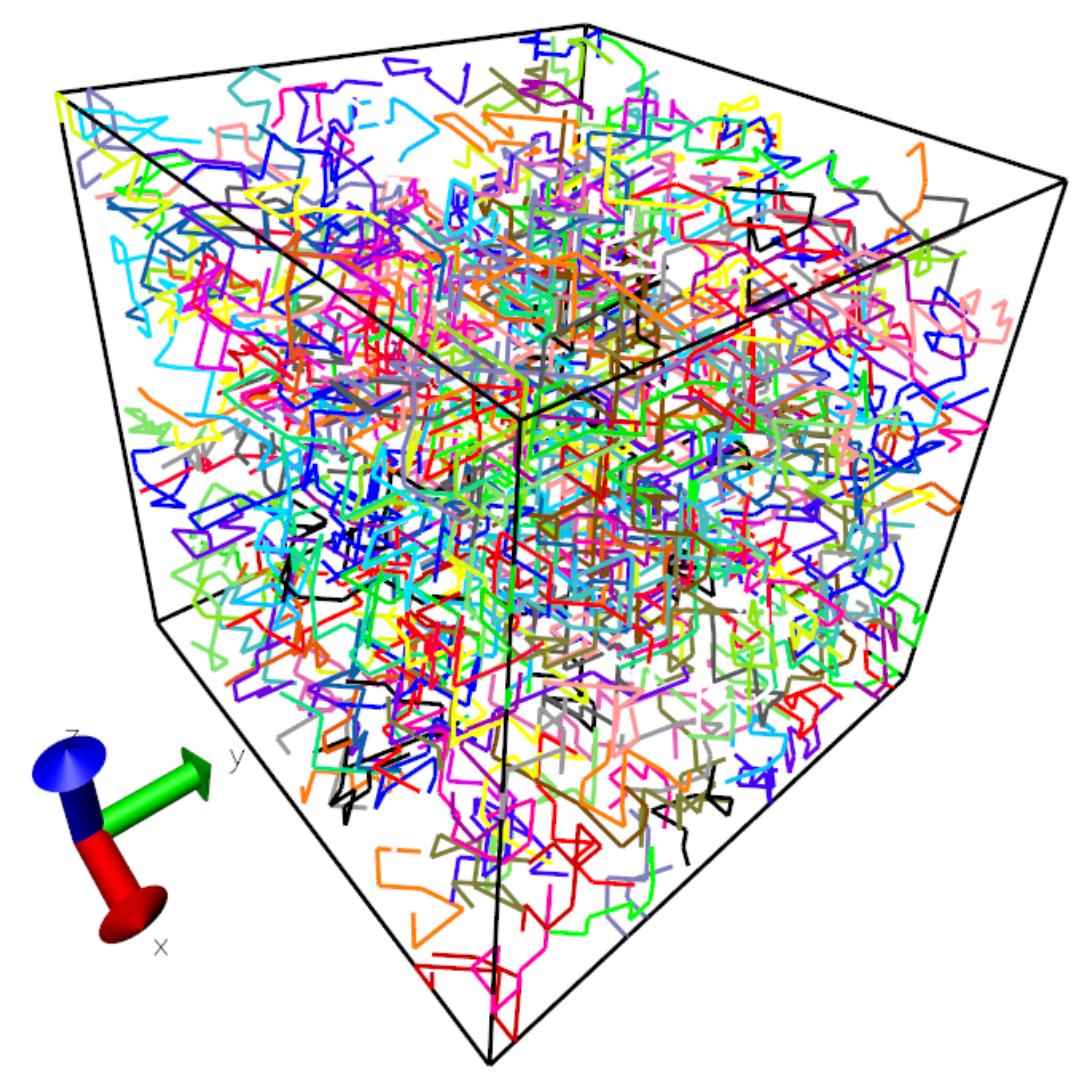

Figure 5: Sensitivity analysis scenario close to the prediction scenario of a polyethylene system. The configuration is generated using the Monte Carlo self-avoiding random walk algorithm within a cube of dimensions $10 \times 10 \times 10 \mathrm{~nm}$. The colored lines represent different chains (total number of chains $=500$, chain length $=10 \mathrm{CG}$ beads). 


\section{BAYESIAN FRAMEWORK FOR MODEL VALIDATION UNDER}

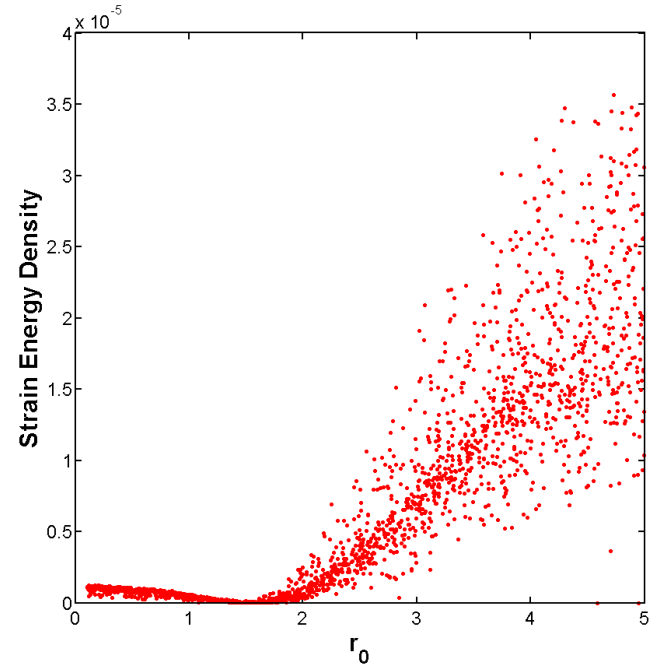

(a)

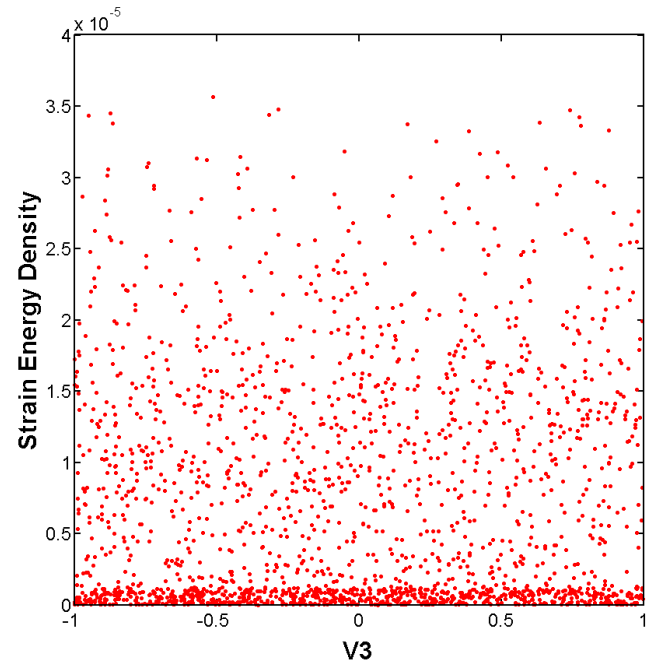

(c)

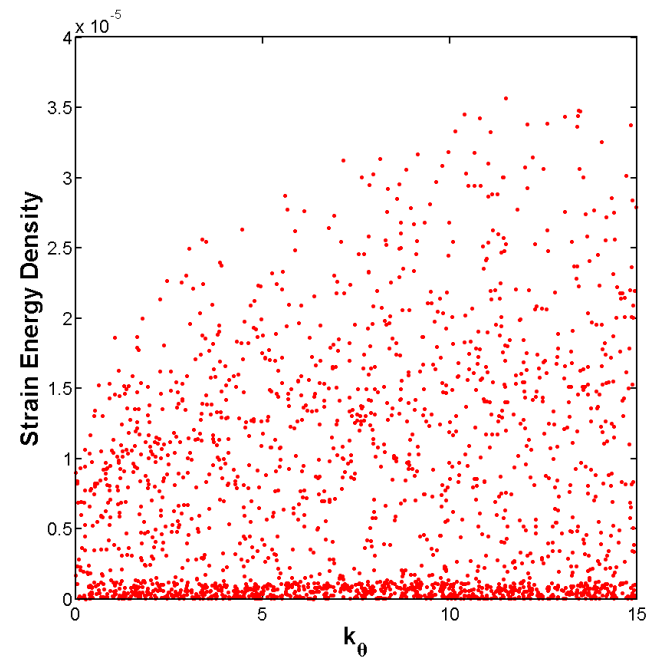

(b)

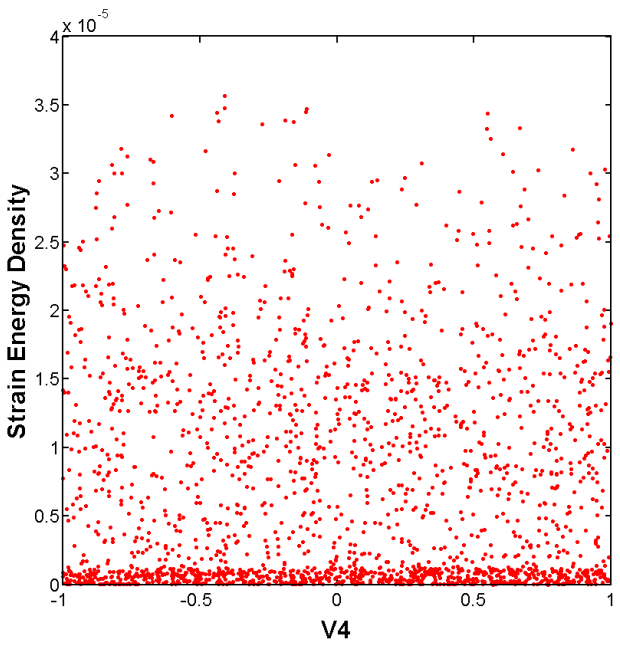

(d)

Figure 6: Scatterplots illustrating a qualitative sensitivity analysis for the prediction scenario of the polyethylene system using 1000 Monte Carlo samples of the parameter space. The observable is the value of the strain-energy density function $W$ at the deformation corresponding to $\lambda_{x}=1.3$.

\subsection{Model Plausibility}

Returning to Bayes's rule (14), and recognizing explicitly that we are calibrating a single model $\mathcal{P}_{j}$ among a set of possible models $\mathcal{M}$, we rewrite the rule in an 


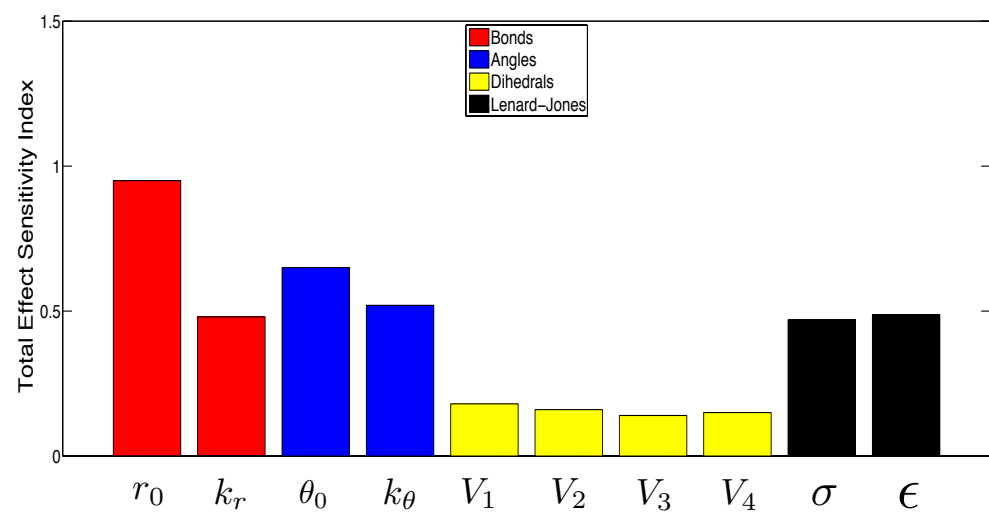

\begin{tabular}{|c|c|}
\hline Parameters & $S_{T}$ \\
\hline \hline$r_{0}$ & 0.95 \\
\hline$k_{r}$ & 0.48 \\
\hline$\theta_{0}$ & 0.65 \\
\hline$k_{\theta}$ & 0.52 \\
\hline$V_{1}$ & 0.18 \\
\hline$V_{2}$ & 0.16 \\
\hline$V_{3}$ & 0.14 \\
\hline$V_{4}$ & 0.15 \\
\hline$\sigma$ & 0.47 \\
\hline$\varepsilon$ & 0.48 \\
\hline
\end{tabular}

Figure 7: Bar chart illustrating the results of the sensitivity analysis using total sensitivity indices for the deformation of the prediction scenario of the polyethylene system using 1000 Monte Carlo samples of the parameter space.

equivalent way that recognizes probabilities conditioned on the choices $\mathcal{P}_{j}$ and $\mathcal{M}$,

$$
\pi\left(\boldsymbol{\theta}_{j} \mid \mathbf{D}_{c}, \mathcal{P}_{j}, \mathcal{M}\right)=\frac{\pi\left(\mathbf{D}_{c} \mid \boldsymbol{\theta}_{j}, \mathcal{P}_{j}, \mathcal{M}\right) \pi\left(\boldsymbol{\theta} \mid \mathcal{P}_{j}, \mathcal{M}\right)}{\pi\left(\mathbf{D}_{c} \mid \mathcal{P}_{j}, \mathcal{M}\right)}, \quad 1 \leq j \leq m
$$

The marginalization of the numerator on the right-hand side is the model evidence,

$$
\pi\left(\mathbf{D}_{c} \mid \mathcal{P}_{j}, \mathcal{M}\right)=\int_{\Theta} \pi\left(\mathbf{D}_{c} \mid \boldsymbol{\theta}, \mathcal{P}_{j}, \mathcal{M}\right) \pi\left(\boldsymbol{\theta} \mid \mathcal{P}_{j}, \mathcal{M}\right) d \boldsymbol{\theta}
$$

The model evidence can be regarded as a likelihood density in a higher form of Bayes's rule, where we compute the posterior model plausibility,

$$
\rho_{j}=\pi\left(\mathcal{P}_{j} \mid \mathbf{D}_{c}, \mathcal{M}\right)=\frac{\pi\left(\mathbf{D}_{c} \mid \mathcal{P}_{j}, \mathcal{M}\right) \pi\left(\mathcal{P}_{j} \mid \mathcal{M}\right)}{\pi\left(\mathbf{D}_{c} \mid \mathcal{M}\right)}, \quad 1 \leq j \leq m
$$

where,

$$
\sum_{j=1}^{m} \rho_{j}=1 .
$$

The model(s) in class $\mathcal{M}$ with plausibilities $\rho_{j}$ closest to one are deemed the most plausible for given data $\mathbf{D}_{c}$. The use of model plausibilies as a basis for model selection has been advocated [5-7, 45, 48, 49] and used successfully in selecting CG models in [21].

\subsection{Validation}

With the calibration posterior $\pi\left(\boldsymbol{\theta} \mid \mathbf{D}_{c}\right)$ for the model judged at this point to be the most plausible in hand, we compute the posterior for a validation scenario with 
validation data $\mathbf{D}_{v}$ :

$$
\pi\left(\boldsymbol{\theta} \mid \mathbf{D}_{v}, \mathbf{D}_{c}\right)=\frac{\pi\left(\mathbf{D}_{v} \mid \boldsymbol{\theta}, \mathbf{D}_{c}\right) \pi\left(\boldsymbol{\theta} \mid \mathbf{D}_{c}\right)}{\pi\left(\mathbf{D}_{v} \mid \mathbf{D}_{c}\right)}
$$

Thus, the validation posterior in (20) represents an update of the calibrated parameters obtained using new data $\mathbf{D}_{v}$ designed to reflect the best representation of the QoI possible in the validation scenario (see, e.g. [21, 48]). While not always necessary, it is possible at this point to compute the information gain,

$$
I\left(\pi\left(\boldsymbol{\theta} \mid \mathbf{D}_{v}, \mathbf{D}_{c}\right), \pi\left(\boldsymbol{\theta} \mid \mathbf{D}_{c}\right)\right)=\int_{\mathbf{D}_{v}} D_{K L}\left(\pi\left(\boldsymbol{\theta} \mid \mathbf{D}_{v}, \mathbf{D}_{c}\right) \| \pi\left(\boldsymbol{\theta} \mid \mathbf{D}_{c}\right)\right) \pi\left(\mathbf{D}_{v}\right) d \mathbf{D}_{v},
$$

as a measure of the value of the validation-test information over that embodied in the calibrations. Here, $D_{K L}(\cdot \| \cdot)$ is the Kullback-Leibler divergence between two distributions $p$ and $q$ defined to be

$$
D_{K L}(p \| q)=\int p(y) \log \frac{p(y)}{q(y)} d y .
$$

With the updated parameters determined by (20), we now solve the forward problem for the validation observable and compute the accuracy of the CG prediction. It is important to note that the observable computed by the CG model depends on the parameters $\boldsymbol{\theta}$. Since the parameters are random variables, so are the observables. Therefore, to solve the forward problem, the validation posterior $\pi\left(\boldsymbol{\theta} \mid \mathbf{D}_{v}, \mathbf{D}_{c}\right)$ is stochastically sampled and a pdf $\pi(U(\boldsymbol{\theta}, G))$ of the observable, for example the potential energy, is produced.

If the target observable, such as the AA potential energy, is a probability distribution, $\pi\left(u_{A A}\right)$, a natural measure of the accuracy of the $\mathrm{CG}$ approximation is the Kullback-Leibler divergence,

$$
\gamma=D_{K L}\left(\pi\left(u_{A A}\right) \| \pi(U(\boldsymbol{\theta}, G))\right)=\int_{\Gamma_{v}} \pi\left(u_{A A}(\omega)\right) \log \frac{\pi\left(u_{A A}(\omega)\right)}{\pi(U(\boldsymbol{\theta}, G(\omega)))} d \omega,
$$

where $\Gamma_{v}$ is the reduced phase space available in the validation scenario. If instead, the target observable is a scalar, usually an ensemble average such as $\left\langle u_{A A}\right\rangle$, the corresponding CG value must be found by taking the expected value,

$$
\langle U\rangle=\mathbb{E}_{\pi_{v}}[\langle U(\boldsymbol{\theta}, G)\rangle]=\int_{\Theta} \pi(U(\boldsymbol{\theta}, G)) \pi\left(\boldsymbol{\theta} \mid \mathbf{D}_{v}, \mathbf{D}_{c}\right) d \boldsymbol{\theta} .
$$

Then the results may be compared in a Euclidean metric,

$$
\gamma=\left|\left\langle u_{A A}\right\rangle-\langle U\rangle\right|
$$

For a preset tolerance $\gamma_{t o l}$, we declare the model valid (technically, "not invalid") if

$$
\gamma \leq \gamma_{t o l}
$$

Otherwise, the model is invalid and we return to the class of models and choose the most plausible in the next level of Occam categories, and repeat the calibration, validation process. This is discussed in detail in Section 4. 


\section{The Occam-Plausibility Algorithm}

Occam's razor is the principle that states that among competing theories that lead to the same prediction, the one that relies on the fewest assumptions is the best. In particular, when choosing among a set of models, the simplest valid model is the best choice. Exactly how to quantify simplicity has been a subject of debate for centuries. Here, the simplicity of a model is assumed to be determined by the number of parameters in the model: a model with two parameters is simpler than a model with three. This is in part due to the complexity and computational cost of solving the inverse problem that yields these parameters. See, for example, Jaynes [33] and Beck and Taflanidis [? ]. Furthermore, the notion of model validity is determined by a Bayesian validation test. With this principle in mind, the following Occam-Plausibility (OP) algorithm is proposed. An example with full detail is given in Section 5.

\section{Initialization}

The algorithm begins when a set $\mathcal{M}$ of possible models is identified. Each model $\mathcal{P}_{j}$ in this set has its own parameters, $\boldsymbol{\theta}_{j}$ so that

$$
\mathcal{M}=\left\{\mathcal{P}_{1}\left(\boldsymbol{\theta}_{1}\right), \ldots, \mathcal{P}_{m}\left(\boldsymbol{\theta}_{m}\right)\right\}
$$

Generally, the models within $\mathcal{M}$ will be closely related; there may be two or more models that have some of the same parameters. For example, in the case of coarsegraining, $\mathcal{M}$ is created by choosing an AA-to-CG map (or several of such maps) and tabulating the possible combinations of types of interactions, as detailed in Section 5 below.

\section{Sensitivity Analysis}

By computing model sensitivities, as described in Section 3.2, a new set of possible models may be defined

$$
\overline{\mathcal{M}}=\left\{\overline{\mathcal{P}}_{1}\left(\overline{\boldsymbol{\theta}}_{1}\right), \ldots, \overline{\mathcal{P}}_{l}\left(\overline{\boldsymbol{\theta}}_{l}\right)\right\}, \quad l \leq m,
$$

where $\overline{\mathcal{P}}_{j}$ are the models in $\mathcal{M}$ that contain only parameters to which the model output is sensitive and models containing output-insensitive parameters have been eliminated.

\section{Occam Step}

After eliminating models that contain insensitive parameters to produce the new set of models $\overline{\mathcal{M}}$, the remaining models can be separated into categories based on the number of model parameters. The models with the least parameters belong 
to Category 1, Category 2 models are slightly more complex and so on. See, for example, Table 1.

In the Occam step, the set

$$
\mathcal{M}^{*}=\left\{\mathcal{P}_{1}^{*}\left(\boldsymbol{\theta}_{1}^{*}\right), \ldots, \mathcal{P}_{k}^{*}\left(\boldsymbol{\theta}_{k}^{*}\right)\right\}
$$

is created such that all of the models $\mathcal{P}_{i}^{*}, 1 \leq i \leq k$ are Category 1 . That is, $\mathcal{M}^{*}$ contains those models in $\overline{\mathcal{M}}$ that have the smallest number of parameters. We begin the calibration and validation cycle (discussed in Section 3) with the simplest model, thus adhering to Occam's Razor.

\section{Calibration and Plausibility Steps}

All of the models in $\mathcal{M}^{*}$ are calibrated via Bayes' rule (16) and their plausibilities (18) are calculated. The most plausible model $\mathcal{P}_{j}^{*}$ may be identified such that

$$
\rho_{i}^{*} \leq \rho_{j}^{*}, \quad i=1, \ldots, k, \quad i \neq j .
$$

Only $\mathcal{P}_{j}^{*}$ will be subjected to the validation test(s).

\section{Validation Step}

The parameters of the most plausible model are updated via Bayes' rule (20), where the observational data $\mathbf{D}_{v}$ comes from the validation scenario. These updated parameters are then used in a forward problem and measured for accuracy via (23) or (25), with validity determined by (26). If the model $\mathcal{P}_{j}^{*}$ is deemed valid, it is suitable for use in the prediction scenario; otherwise, the model is invalid. Once a valid model is identified, the $(\mathrm{OP})$ algorithm is complete. Otherwise the algorithm proceeds to the iterative Occam step.

\section{Iteration}

If $\mathcal{P}_{j}^{*}$ is invalid, $\mathcal{M}^{*}$ is redefined so as to contain models of the next category. If $\mathcal{P}_{j}^{*}$ is Category 1 , the new $\mathcal{M}^{*}$ would contain Category 2 models, and so on. One additional constraint that may be imposed is to mandate that all of the models in the new $\mathcal{M}^{*}$ contain all of the parameters in $\mathcal{P}_{j}^{*}$ so that they build on the information learned about the preference the system has about model parameters.

If this iteration has been repeated enough times so that $\mathcal{P}_{j}^{*}$ already belongs to the highest category of models in $\overline{\mathcal{M}}$, the modeler must define a new set of models $\mathcal{M}$, conduct a new sensitivity analysis, and iterate through the algorithm until a valid model is found. A flowchart summarizing the full OP algorithm is shown in Figure 8 . 


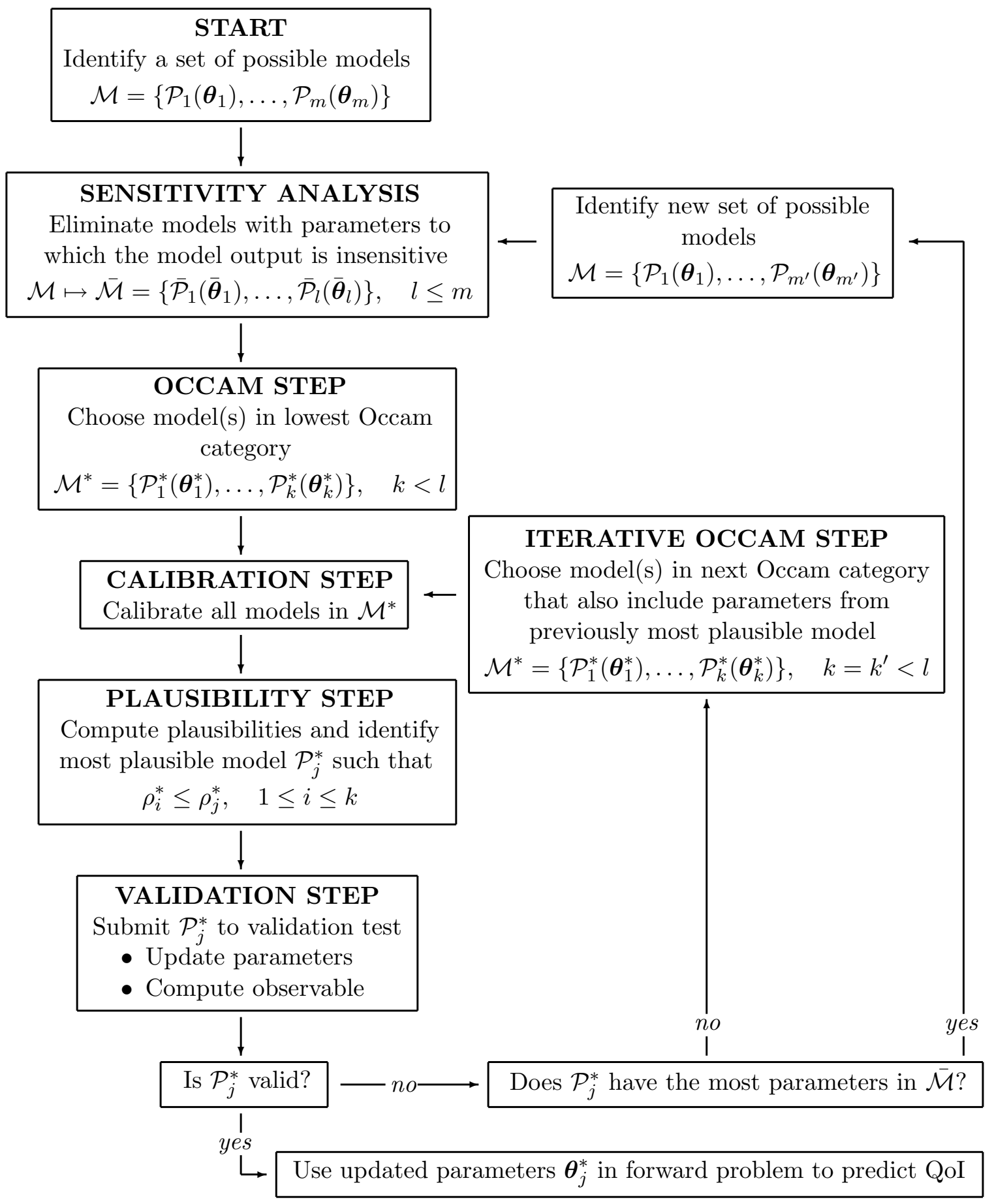

Figure 8: Flowchart of the Occam-Plausibility Algorithm. 


\section{$5 \quad$ An Example Application}

We return to the application of coarse graining to polyethylene to illustrate the OP Algorithm. Recall that in this example, to coarse grain polyethylene, two carbon atoms and their accompanying hydrogen atoms are aggregated into a single coarse-grained particle. The coarse-grained system of polyethylene has one type of particle, yielding only one type of bond, one type of angle, and one type of dihedral interaction.

\section{Initialization}

As mentioned previously, the set of coarse-grained models $\mathcal{M}=\left\{\mathcal{P}_{1}\left(\boldsymbol{\theta}_{1}\right), \ldots\right.$, $\left.\mathcal{P}_{23}\left(\boldsymbol{\theta}_{23}\right)\right\}$ is created by tabulating the possible combinations of interactions, which are listed in Table 1. Note that the Lennard-Jones 9-6 potential has been added as an alternative to the Lennard-Jones 12-6. The LJ 9-6 is a softer potential to account for the empty space that results from grouping atoms into a single sphere, which is an idealization of the CG particle. Furthermore, previous studies have indicated that an additive constant parameter should be added to the potential [21]. This parameter, $A$, represents the potential energy lost due to the coarse graining process and can be thought of as the internal bead energy.

\section{Sensitivity Analysis}

As an observable, we consider the change in the potential energy of a system of polyethylene as a load is applied to it. Data can be collected from the AA version of the system and is compared to the corresponding CG representation, as described in Section 3.2. In the case of calibration, the external force is applied to a chain of 24 carbon atoms and $12 \mathrm{CG}$ particles in the AA and CG systems, respectively. With this in mind, a sensitivity analysis is performed, with the output function $Y$ being the change in potential energy as the chain is stretched. It was shown that of the ten possible parameters, the dihedral parameters are the least influential to the chosen observable. All models with dihedral parameters are consequently removed from the set of possible models, leaving $\overline{\mathcal{M}}=\left\{\overline{\mathcal{P}}_{1}, \ldots, \overline{\mathcal{P}}_{11}\right\}=\left\{\mathcal{P}_{1}, \ldots, \mathcal{P}_{9}, \mathcal{P}_{11}, \mathcal{P}_{12}\right\}$.

\section{Occam Step}

The remaining models are separated into categories according to the number of model parameters. Category 1 contains models $\overline{\mathcal{P}}_{1}, \overline{\mathcal{P}}_{2}, \overline{\mathcal{P}}_{3}$, and $\overline{\mathcal{P}}_{4}$, each of which have two parameters. Models with four parameters, $\overline{\mathcal{P}}_{5}, \overline{\mathcal{P}}_{6}, \overline{\mathcal{P}}_{7}, \overline{\mathcal{P}}_{8}$, and $\overline{\mathcal{P}}_{9}$, are classified as Category 2. The Category 3 models, $\overline{\mathcal{P}}_{10}$ and $\overline{\mathcal{P}}_{11}$, have six parameters each.

In the first Occam Step, only Category 1 models are considered so that

$$
\mathcal{M}^{*}=\left\{\mathcal{P}_{1}^{*}\left(\boldsymbol{\theta}_{1}^{*}\right), \ldots, \mathcal{P}_{4}^{*}\left(\boldsymbol{\theta}_{4}^{*}\right)\right\},
$$




\begin{tabular}{|c|c|c|c|c|c|c|c|}
\hline Model & Bonds & Angles & Dihedrals & LJ 12-6 & LJ 9-6 & Param & Cat. \\
\hline $\mathcal{P}_{1}$ & $\checkmark$ & & & & & 3 & \multirow[t]{4}{*}{1} \\
\hline $\mathcal{P}_{2}$ & & $\checkmark$ & & & & 3 & \\
\hline $\mathcal{P}_{3}$ & & & & $\checkmark$ & & 3 & \\
\hline $\mathcal{P}_{4}$ & & & & & $\checkmark$ & 3 & \\
\hline $\mathcal{P}_{5}$ & $\checkmark$ & $\checkmark$ & & & & 5 & \multirow[t]{6}{*}{2} \\
\hline $\mathcal{P}_{6}$ & $\checkmark$ & & & $\checkmark$ & & 5 & \\
\hline $\mathcal{P}_{7}$ & $\checkmark$ & & & & $\checkmark$ & 5 & \\
\hline $\mathcal{P}_{8}$ & & $\checkmark$ & & $\checkmark$ & & 5 & \\
\hline $\mathcal{P}_{9}$ & & $\checkmark$ & & & $\checkmark$ & 5 & \\
\hline $\mathcal{P}_{10}$ & . & & $\checkmark$ & & & 5 & \\
\hline $\mathcal{P}_{11}$ & $\checkmark$ & $\checkmark$ & & $\checkmark$ & & 7 & \multirow[t]{6}{*}{3} \\
\hline $\mathcal{P}_{12}$ & $\checkmark$ & $\checkmark$ & & & $\checkmark$ & 7 & \\
\hline $\mathcal{P}_{13}$ & $\checkmark$ & & $\checkmark$ & & & 7 & \\
\hline $\mathcal{P}_{14}$ & & $\checkmark$ & $\checkmark$ & & & 7 & \\
\hline $\mathcal{P}_{15}$ & & & $\checkmark$ & $\checkmark$ & & 7 & \\
\hline $\mathcal{P}_{16}$ & & & $\checkmark$ & & $\checkmark$ & 7 & \\
\hline $\mathcal{P}_{17}$ & $\checkmark$ & $\checkmark$ & $\checkmark$ & & & 9 & \multirow[t]{5}{*}{4} \\
\hline $\mathcal{P}_{18}$ & $\checkmark$ & & $\checkmark$ & $\checkmark$ & & 9 & \\
\hline $\mathcal{P}_{19}$ & $\checkmark$ & & $\checkmark$ & & $\checkmark$ & 9 & \\
\hline $\mathcal{P}_{20}$ & & $\checkmark$ & $\checkmark$ & $\checkmark$ & & 9 & \\
\hline $\mathcal{P}_{21}$ & & $\checkmark$ & $\checkmark$ & & $\checkmark$ & 9 & \\
\hline $\mathcal{P}_{22}$ & $\checkmark$ & $\checkmark$ & $\checkmark$ & $\checkmark$ & & 11 & \multirow[t]{2}{*}{5} \\
\hline $\mathcal{P}_{23}$ & $\checkmark$ & $\checkmark$ & $\checkmark$ & & $\checkmark$ & 11 & \\
\hline
\end{tabular}

Table 1: Table of possible CG models created by all combinations of interaction potentials that may be included in the representation of the potential energy along with the number of parameters they contain (including $A$ ) and their Occam categories.

where $\mathcal{P}_{i}^{*}=\overline{\mathcal{P}}_{i}$. That is, $\mathcal{P}_{1}^{*}$ contains only bonded interactions with $\boldsymbol{\theta}_{1}^{*}=\left\{r_{0}, k_{r}\right\}$; $\mathcal{P}_{2}^{*}$ has only angular interactions, where $\boldsymbol{\theta}_{2}^{*}=\left\{\theta_{0}, k_{\theta}\right\}$; and $\mathcal{P}_{3}^{*}$ and $\mathcal{P}_{4}^{*}$ only account for Lennard-Jones non-bonded interactions with $\boldsymbol{\theta}_{3}^{*}=\boldsymbol{\theta}_{4}^{*}=\{\sigma, \epsilon\}$, though the representations differ slightly as previously mentioned.

\section{Calibration and Plausibility Steps}

Each of these models is calibrated using a Gaussian likelihood distribution with $\mathbf{D}=\left\{D_{i}\right\}_{i=1}^{150}=\left\{u\left(\omega_{i}\right)\right\}_{i=1}^{150}$, where $u\left(\omega_{i}\right)$ is the potential energy of a single hexane molecule in configuration $\omega_{i}$, and $\mathbf{d}(\boldsymbol{\theta})=\left\{U\left(G\left(\omega_{i}\right), \boldsymbol{\theta}\right)\right\}_{i=1}^{150}$, where $U$ is the CG potential energy and $G\left(\omega_{i}\right)$ is the CG representation of the AA configuration $\omega_{i}$. Priors for parameters are computed using the maximum entropy method on statis- 
tical data gathered from the AA system, as detailed in the Appendix of [21], yielding the prior distribution functions $\pi\left(\boldsymbol{\theta}_{i}^{*} \mid \mathcal{P}_{i}^{*}, \mathcal{M}\right)$. After all four models are calibrated, the following plausibilities are calculated using (18),

$$
\rho_{1}^{*}=1, \quad \rho_{2}^{*} \approx 0, \quad \rho_{3}^{*} \approx 0, \quad \rho_{4}^{*} \approx 0,
$$

illustrating $\mathcal{P}_{1}^{*}$ is the most (and the only) plausible model.

\section{Validation Step}

Moving into the validation step, the calibration posterior $\pi\left(\boldsymbol{\theta}_{1}^{*} \mid \mathbf{D}, \mathcal{P}_{1}^{*}, \mathcal{M}^{*}\right)$ becomes the prior in the validation scenario, where we consider a canonical ensemble simulation of a single molecule of octadecane. Again, the likelihood is taken to be Gaussian with $\mathbf{D}_{v}=\left\{D_{i}\right\}_{i=1}^{150}=\left\{u\left(\omega_{i}\right)\right\}_{i=1}^{150}$, where $u\left(\omega_{i}\right)$ is the potential energy of the system in configuration $\omega_{i}$, and $\mathbf{d}(\boldsymbol{\theta})=\left\{U\left(G\left(\omega_{i}\right), \boldsymbol{\theta}\right)\right\}_{i=1}^{150}$, where $U$ is the CG potential energy of the corresponding CG configuration $G\left(\omega_{i}\right)$. The parameters for $\mathcal{P}_{1}^{*}$ are updated via $(20)$, and the updated parameters are used to simulate a CG system of octadecane.

For the validation observable, we use the potential energy of the system so that $\pi\left(Q \mid \boldsymbol{\theta}_{1, v}^{*}\right)=\pi\left(U\left(G, \boldsymbol{\theta}_{1, v}^{*}\right)\right)$ if the observable is the entire distribution of the potential energy or, if a scalar is selected, $Q=\left\langle U\left(G, \boldsymbol{\theta}_{1, v}^{*}\right)\right\rangle$. The distributions of the potential energy of the CG and AA systems of octadecane are shown in Fig. 9. From this it can be qualitatively seen that the model is not valid.

Quantitatively, we select the validation criteria for the scalar and pdf observables, respectively, to be

$$
\gamma_{1, t o l}=0.2 Q_{A A}, \quad \gamma_{2, t o l}=0.05 \sigma_{A A}^{2},
$$

where $Q_{A A}=\left\langle u_{A A}\right\rangle$ and $\sigma_{A A}^{2}$ is the variance of $\pi\left(u_{A A}\right)$. Using (23) and (25), we obtain

$$
\gamma_{1}=0.6173 Q_{A A}, \quad \gamma_{2}=0.2435 \sigma_{A A}^{2},
$$

rendering $\mathcal{P}_{1}^{*}$ invalid as $\gamma_{1}>\gamma_{1, \text { tol }}$ and $\gamma_{2}>\gamma_{2, t o l}$.

\section{Iteration}

Moving into the iterative Occam step, a new $\mathcal{M}^{*}$ is defined which contains models in the next category, in this case is Category 2. In addition, we impose the constraint that the models in this set contain the parameters that were included in the previously most-plausible model. That is, models in $\mathcal{M}^{*}$ must contain bond parameters. This constraint creates an algorithm that is closer to hierarchical modeling in which the most plausible model is built upon and improved instead of discarded when invalidated.

The new $\mathcal{M}^{*}$ is therefore

$$
\mathcal{M}^{*}=\left\{\mathcal{P}_{1}^{*}\left(\boldsymbol{\theta}_{1}^{*}\right), \mathcal{P}_{2}^{*}\left(\boldsymbol{\theta}_{2}^{*}\right), \mathcal{P}_{3}^{*}\left(\boldsymbol{\theta}_{3}^{*}\right)\right\},
$$


Distributions of AA and CG Potential Energies of $S_{v}$

Distributions of $A A$ and $C G$ Potential Energies of $S_{v}$

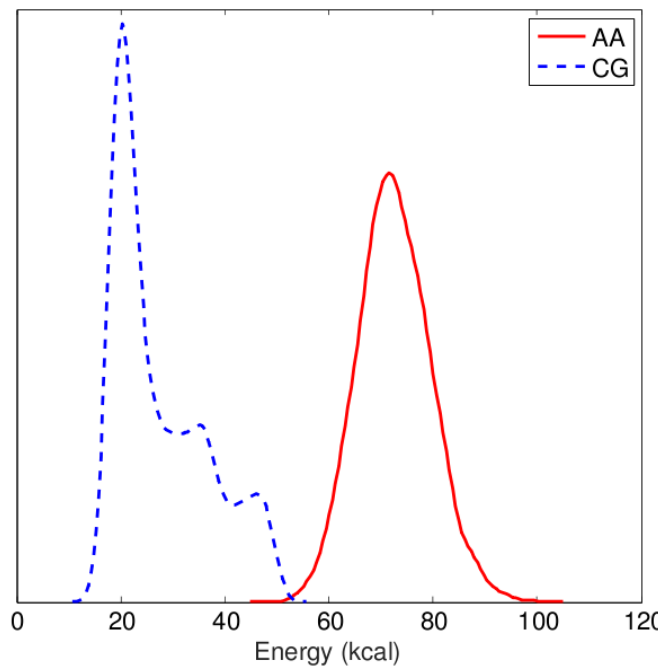

(a)

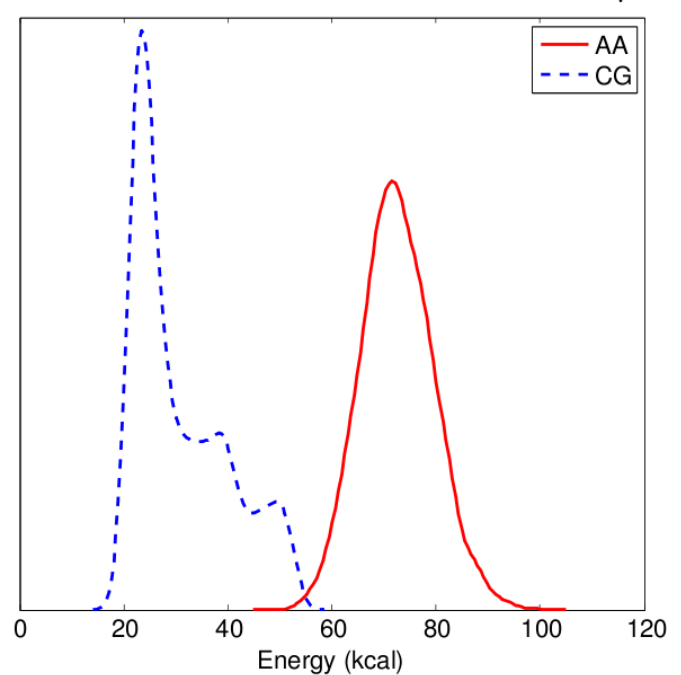

(b)

Figure 9: Comparison of the AA and CG distributions of the validation observable. The CG observables are computed using the parameter distributions, $\pi\left(\boldsymbol{\theta} \mid \mathbf{D}_{v}, \mathbf{D}_{c}\right)$, yielded by the validation update from the first Occam level (a) and the second Occam level (b). Qualitatively, it can be seen that the CG models are not valid.

where $\mathcal{P}_{1}^{*}=\overline{\mathcal{P}}_{5}$ contains bonded and angular interactions with $\boldsymbol{\theta}_{1}^{*}=\left\{k_{r}, r_{0}, k_{\theta}, \theta_{0}\right\}$, $\mathcal{P}_{2}^{*}=\overline{\mathcal{P}}_{6}$ considers bonded and LJ 12-6 interactions so that $\boldsymbol{\theta}_{2}^{*}=\left\{k_{r}, r_{0}, \sigma, \epsilon\right\}$, and $\mathcal{P}_{3}^{*}=\overline{\mathcal{P}}_{7}$ has bonded and LJ 9-6 interactions with $\boldsymbol{\theta}_{3}^{*}=\left\{k_{r}, r_{0}, \sigma, \epsilon\right\}$.

Considering the same calibration scenario described above, each model in $\mathcal{M}^{*}$ is calibrated. The ensuing plausibility calculations yield,

$$
\rho_{1}^{*}=3.7891 \times 10^{-7}, \quad \rho_{2}^{*}=0.3420, \quad \rho_{3}^{*}=0.6580,
$$

making $\mathcal{P}_{3}^{*}$ the most plausibile model with $\mathcal{P}_{2}^{*}$ close behind. Considering also the same validation scenario, the parameters of $\mathcal{P}_{3}^{*}$ are updated and the same validation test is performed. The resulting distributions of the potential energy are shown in Fig. 9. Using the same observables, $\pi\left(Q \mid \boldsymbol{\theta}_{3, v}^{*}\right)=\pi\left(U\left(G, \boldsymbol{\theta}_{3, v}^{*}\right)\right)$ and $Q=\left\langle U\left(G, \boldsymbol{\theta}_{3, v}^{*}\right)\right\rangle$, we find

$$
\gamma_{1}=0.5731 Q_{A A}, \quad \gamma_{2}=0.2084 \sigma_{A A}^{2}
$$

by (25) and (23), respectively. Thus $\mathcal{P}_{3}^{*}$ is invalid by criteria (33).

We again move through the iterative Occam step, where $\mathcal{M}^{*}$ now contains models of Category 3 that include parameters from the previous $\mathcal{P}_{3}^{*}$. That is,

$$
\mathcal{M}^{*}=\left\{\mathcal{P}_{1}^{*}\left(\boldsymbol{\theta}_{1}^{*}\right), \mathcal{P}_{2}^{*}\left(\boldsymbol{\theta}_{2}^{*}\right)\right\},
$$


Distributions of AA and CG Potential Energies of $S_{v}$

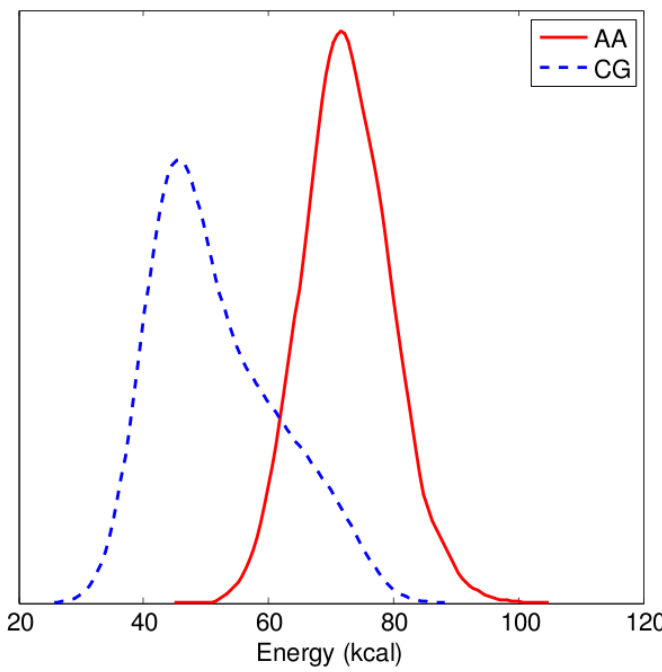

(a)
Distributions of AA and CG Potential Energies of $S_{v}$

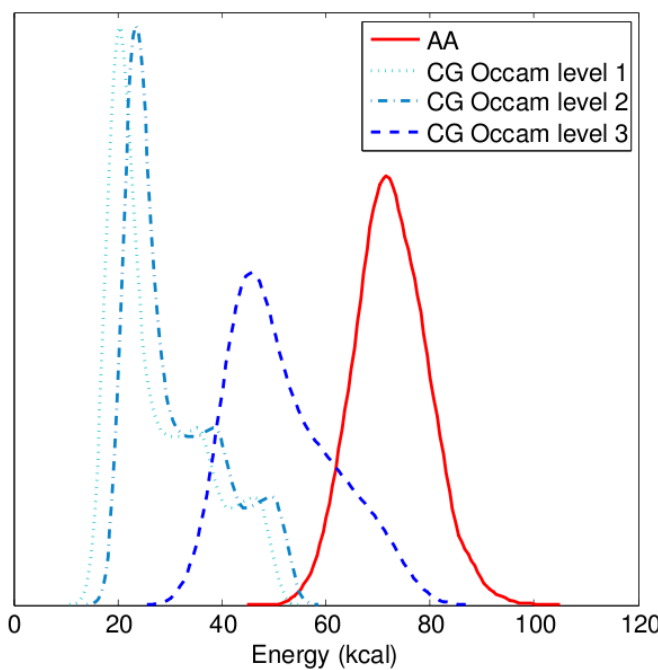

(b)

Figure 10: Comparison of the AA and CG distributions of the validation observable. The CG observables are computed using the parameter distributions, $\pi\left(\boldsymbol{\theta} \mid \mathbf{D}_{v}, \mathbf{D}_{c}\right)$, yielded by the validation update from the last Occam level (a). In (b), we see that the $\mathrm{CG}$ distributions get closer to the AA distribution with each iteration of the Occam-Plausibility Algorithm.

where $\mathcal{P}_{1}^{*}=\overline{\mathcal{P}}_{10}$ includes bonded, angular, and LJ 12-6 interactions and $\mathcal{P}_{2}^{*}=\overline{\mathcal{P}}_{11}$ includes bonded, angular, and LJ 9-6 interactions. Both models have the same parameter vector, $\boldsymbol{\theta}_{j}^{*}=\left\{k_{r}, r_{0}, k_{\theta}, \theta_{0}, \sigma, \epsilon\right\}$. Calibration and plausibility calculations show

$$
\rho_{1}^{*}=0.5, \quad \rho_{2}^{*}=0.5
$$

making both models equally plausible. Performing the validation update and validation test, the results of which are shown in Fig. 10, we calculate

$$
\gamma_{1}=0.1721 Q_{A A}, \quad \gamma_{2}=0.0452 \sigma_{A A}^{2} .
$$

Therefore, $\gamma_{1} \leq \gamma_{1, t o l}$ and $\gamma_{2} \leq \gamma_{2, t o l}$, making model $\mathcal{P}_{1}^{*}$ valid according to the validation criteria and observables selected.

It can be argued that, qualitatively, the Category 3 model deemed valid according to the preset validation criteria is not "close enough" to the observable produced by the AA model. Thus we may wish to tighten our validation criteria to, for example,

$$
\gamma_{2, t o l}=0.1 Q_{A A} .
$$

In this case, the model $\mathcal{P}_{1}^{*}$ from Category 3 is no longer valid. Since this model has the most parameters in $\overline{\mathcal{M}}$, following the OP Algorithm requires identifying a 
completely new set, $\mathcal{M}$, of possible models. This may constitute identifying new functional forms that may possibly represent the physics of the system (in this case, new functional forms for the potential energy $U$ ) or identifying a new degree-offreedom reduction (in this case, a new AA-to-CG map).

Thus, let us redefine our coarse-grained mapping so that each CG particle contains a single carbon atom and the hydrogen atoms that are bonded to it. This representation is often called the united atom (UA) representation. As before, this coarse-grained system of polyethylene has one type of particle, yielding the same set of models and the same Occam categories listed in Table 1. A sensitivity study is carried out in the prediction $\left(\mathrm{C}_{24} \mathrm{H}_{50}\right)$, validation $\left(\mathrm{C}_{18} \mathrm{H}_{38}\right)$, and calibration $\left(\mathrm{C}_{6} \mathrm{H}_{14}\right)$ scenarios. Again, dihedral interactions may be disregarded.

Starting again with the models in Category 1, the same calibration scenario previously described yields plausibilities

$$
\rho_{1}^{*}=1, \quad \rho_{2}^{*} \approx 0, \quad \rho_{3}^{*} \approx 0, \quad \rho_{4}^{*} \approx 0 .
$$

Thus model $\mathcal{P}_{1}^{*}$, which contains only bonded interactions, is moved into the validation sstage, where the same validation scenario is used. The observables produced by the updated parameters are shown in Figure 11. Using 25,

$$
\gamma_{2}=0.08 Q_{A A},
$$

rendering $\mathcal{P}_{1}^{*}$ valid for predicting the chosen quantity of interest.

\section{Summary and Conclusions}

Bayesian statistical inverse analysis can provide a powerful approach to the calibration and validation of coarse-grained models of atomistic systems, an approach that inherently copes with uncertainties in model parameters, data, and even the selection of the model itself.

Each family of CG models is perceived to be the result of a map $G$ from the AA system to the CG configurations, and each image under the map corresponds to a set of parametric classes of models differing in the conformation of potential energy terms and in model parameters. There can be many maps $G\left(=G_{1}, G_{2}, \cdots, G_{k}\right)$, each corresponding to a decision on the structure of the CG model.

In many cases, key observables and quantities of interest to be predicted by CG models are not sensitive to changes in model parameters. The calculation of variance-based model sensitivities provides an a priori indication of which parameters do and do not influence predictions, and those that do not, can, in theory, be eliminated at the onset. A more qualitative indication of parameter sensitivities can also be provided by scatterplots, which can be used in conjunction with sensitivity calculations. We provided examples of cases in which sensitivities computed for both calibration and prediction scenarios produced consistent indications of parameters that could be removed without appreciably influencing predicted observables. 


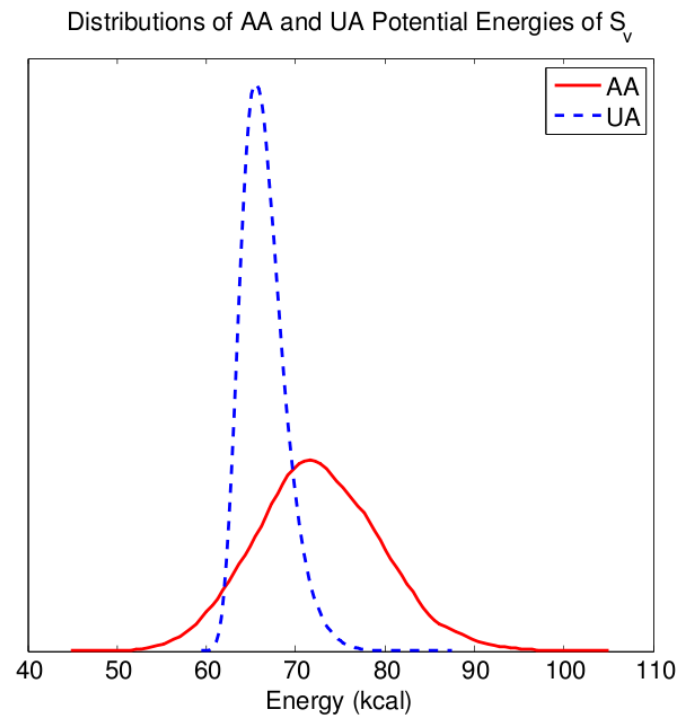

Figure 11: Comparison of the AA and CG distributions of the validation observable. The CG observable is computed using the UA model and the parameters deemed "valid" according to the validation criteria (43).

Once a sensitivity analysis is implemented and irrelevant parameters are discarded, one can partition the surviving model classes into a hierarchy we refer to as Occam categories, in deference to Occam's razor, with model simplicity equated to the number of parameters in each model class. We, therefore, need to use only models in lowest category containing a valid model: once a valid model class is identified, it is not necessary to consider other models which, by construction, have more than the minimum number of required parameters. We calibrate these models in a calibration scenario, as discussed in Section 3.2. But which models among a calibrated category are best?

The notion of Bayesian posterior model plausibilities can greatly reduce the ambiguity in model selection and can provide a systematic basis for model selection among classes of $\mathrm{CG}$ models for given AA data. The most plausible model in a sensitivity class is determined using (18) and (19) for given AA data.

Is the most plausible model in a category valid? To answer this question requires that we construct a validation scenario (or experiment), select a meaningful AA observable and, importantly, select a tolerance that we set as the tolerable accuracy we will accept for the models's ability to predict the observable. We impose conditions such as (23), (25), and (26). If these criteria are met, the model is declared "valid", even though we understand that the choice of the observable, the means to measure prediction accuracy, and the chance of the tolerance $\gamma$ are subjective choices: different choices may lead to the conclusion that the model is 
invalid (hence, one sometimes claims a model is "not invalid" as a weaker conclusion than the model is "valid").

If the model is invalid, we must move to a higher Occam category of models. If models in all categories prove to be invalid, we must redefine the AA-to-CG map, going to another family of model classes. The choice of a $G_{k}$ more likely to succeed is, at this point, a subjective decision, based largely on prior information and the experience of the modeler.

We refer to the process just described and demonstrated in this work as the Occam-Plausibility Algorithm. Several possibilities for improvement and refinement of steps in the process exist, and will hopefully be the subject of future work. We contemplate extending our validation algorithm to truly multiscale models in which valid CG models are used as a basis for virtual validation of macroscale models of continua. We hope to also check such results with appropriate experimental data if accessible.

\section{Acknowledgments}

This material is based upon work supported by the U.S. Department of Energy Office of Science, Office of Advanced Scientific Computing Research, Applied Mathematics program under Award Number DE-5C0009286. We like to acknowledge the many fruitful discussions with Ron Elber, Peter Rossky, and Eric Wright, from which we've benefited greatly, and earlier interactions with Serge Prudhomme.

\section{References}

[1] M. Adams, D. Higdon, et al., editors. Assessing the Reliability of Complex Models: Mathematical and Statistical Foundations of Verification, Validation, and Uncertainty Quantification. The National Academic Press, Washington, D.C., 2012.

[2] P. Angelikopoulos, C. Papadimitriou, and P. Koumoutsakos. Bayesian uncertainty quantification and propagation in molecular dynamics simulations: A high performance computing framework. The Journal of Chemical Physics, 137(14):-, 2012.

[3] P. Angelikopoulos, C. Papadimitriou, and P. Koumoutsakos. Data driven, predictive molecular dynamics for nanoscale flow simulations under uncertainty. The Journal of Physical Chemistry B, 117:14808-14816, 2013.

[4] I. Babuška, R. Tempone, and F. Nobile. A systematic approach to model validation based on Bayesian updates and prediction-related rejection criteria. Computer Methods in Applied Mechanics and Engineering, 197:2517-2539, 2008. 
[5] J. L. Beck. Bayesian system identification based on probability logic. Structural Control and Health Monitoring, 17(7):825-847, 2010.

[6] J. L. Beck and A. A. Taflanidis. Prior and posterior robust stochastic predictions for dynamical systems using probability logic. International Journal for Uncertainty Quantification, 3(4):271-288, 2013.

[7] J. L. Beck and K.-V. Yuen. Model selection using response measurements: Bayesian probabilistic approach. Journal of Engineering Mechanics, 130(2):192-203, 2004.

[8] K. Binder. Monte Carlo and Molecular Dynamics Simulations in Polymer Science. Oxford Univ. Press, New York, 1995, 1995.

[9] E. Brini, V. Marcon, and N. F. A. van der Vegt. Conditional reversible work method for molecular coarse graining applications. 13(22):10468-10474, 2011.

[10] E. Brini and N. F. A. van der Vegt. Chemically transferable coarse-grained potentials from conditional reverse work calculations. The Journal of Chemical Physics, 137(15):154113, 2012.

[11] B. R. Brooks, R. E. Bruccoleri, B. D. Olafson, D. J. States, S. Swaminathan, and M. Karplus. CHARMM: A program for macromolecular energy, minimization and dynamics calculations. Journal of Computational Chemistry, 4(2):187$217,1983$.

[12] S. P. Carmichael and M. S. Shell. A new multiscale algorithm and its application to coarse-grained peptide models for self-assembly. The Journal of Physical Chemistry B, 116(29):8383-8393, 2012.

[13] A. Chaimovich and M. S. Shell. Coarse-graining errors and numerical optimization using a relative entropy framework. The Journal of Chemical Physics, 134(9):094112, 2011.

[14] W. D. Cornell, P. Cieplak, C. I. Bayly, I. R. Gould, K. M. Merz, Jr., D. M. Ferguson, D. C. Spellmeyer, T. Fox, J. W. Caldwell, and P. A. Kollman. A second generation force field for the simulation of proteins, nucleic acids, and organic molecules. Journal of the American Chemical Society, 117(19):51795197, 1995.

[15] R. I. Cukier, C. M. Fortuin, K. E. Shuler, A. G. Petschek, and J. H. Schaibly. Study of the sensitivity of coupled reaction systems to uncertainties in rate coefficients. i theory. The Journal of Chemical Physics, 59(8):3873-3878, 1973.

[16] A. Das and H. C. Andersen. The multiscale coarse-graining method. ix. a general method for construction of three body coarse-grained force fields. The Journal of Chemical Physics, 136(19):-, 2012. 
[17] A. Das and H. C. Andersen. The multiscale coarse-graining method. viii. multiresolution hierarchical basis functions and basis function selection in the construction of coarse-grained force fields. The Journal of Chemical Physics, 136(19):-, 2012.

[18] F. Ercolessi and J. B. Adams. Interatomic potentials from first-principles calculations: The force-matching method. Europhysics Letters, 26(8):583, 1994.

[19] P. Español and I. Zúñiga. Obtaining fully dynamic coarse-grained models from md. Phys. Chem. Chem. Phys., 13:10538-10545, 2011.

[20] K. Farrell and J. T. Oden. Statistical calibration and validation methods of coarse-grained and macro models of atomic systems. ICES REPORT 12-45, 2012 .

[21] K. Farrell and J. T. Oden. Calibration and validation of coarse-grained models of atomic systems: Application to semiconductor manufacturing. Comput. Mech., 54(1):3-19, July 2014.

[22] P. J. Flory. Thermodynamics of high polymer solutions. The Journal of Computational Physics, 10(1):51-61, 1942.

[23] T. Homma and A. Saltelli. Importance measures in global sensitivity analysis of nonlinear models. Reliability Engineering and System Safety, 52(1):1 - 17, 1996.

[24] D. Hossain, M. Tschopp, D. Ward, J. Bouvard, P. Wang, and M. Horstemeyer. Molecular dynamics simulations of deformation mechanisms of amorphous polyethylene. Polymer, 51(25):6071 - 6083, 2010.

[25] M. L. Huggins. Solutions of long chaim compounds. The Journal of Chemical Physics, 9(5):440, 1941.

[26] S. Izvekov. Towards an understanding of many-particle effects in hydrophobic association in methane solutions. The Journal of Chemical Physics, 134(3):034104, 2011.

[27] S. Izvekov, P. W. Chung, and B. M. Rice. The multiscale coarse-graining method: Assessing its accuracy and introducing density dependent coarsegraining potentials. The Journal of Chemical Physics, 133(6):064109, 2010.

[28] S. Izvekov, M. Parrienllo, C. J. Burnham, and G. A. Voth. Effective force fields for condensed phase systems from ab initio molecular dynamics simulation: A new method for force matching. The Journal of Chemical Physics, 120(23):10896-10913, 2004. 
[29] S. Izvekov and G. A. Voth. A multiscale coarse-graining method for biomolecular systems. The Journal of Physical Chemistry B, 109(7):2469-2473, 2005.

[30] S. Izvekov and G. A. Voth. Multiscale coarse graining of liquid-state systems. The Journal of Chemical Physics, 123(13):134105, 2005.

[31] S. Izvekov and G. A. Voth. Modeling real dynamics in the coarse-grained representation of condensed phase systems. The Journal of Chemical Physics, 125(15):151101, 2006.

[32] E. T. Jaynes. Prior probabilities. Systems Science and Cybernetics, IEEE Transactions on, 4(3):227-241, 1968.

[33] E. T. Jaynes. Probability Theory: The Logic of Science. Cambridge University Press, Cambridge, 2003.

[34] W. L. Jorgensen, D. S. Maxwell, and J. Tirado-Rives. Development and testing of the OPLS all-atom force field on conformational energetics and properties of organic liquids. Journal of the American Chemical Society, 118(45):1122511236, 1996.

[35] W. L. Jorgensen and J. Tirado-Rives. The OPLS potential functions for proteins. Energy minimizations for crystals of cyclic peptides and crambin. Journal of the American Chemical Society, 110(6):1657-1666, 1988.

[36] Y. Li, B. C. Abberton, M. Kröger, and W. K. Liu. Challenges in multiscale modeling of polymer dynamics. Polymers, 5(2):751-832, 2013.

[37] P. Liu, Q. Shi, H. Daumé, and G. A. Voth. A bayesian statistic approach to multiscale coarse graining. The Journal of Chemical Physics, 129(21):214114, 2008.

[38] A. P. Lyubartsev and A. Laaksonen. Calculation of effective interaction potentials from radial distribution functions: A reverse Monte Carlo approach. Physical Review E, 52(4):3730-3737, 1995.

[39] A. P. Lyubartsev and A. Laaksonen. Effective potentials for ion-DNA interactions. The Journal of Chemical Physics, 111(24):11207, 1999.

[40] R. L. McGreevy and L. Pusztai. Reverse Monte Carlo simulation: A new technique for the determination of disordered structures. Molecular Simulation, 1(6):359-367.

[41] J. W. Mullinax and W. G. Noid. Extended ensemble approach for deriving transferable coarse-grained potentials. The Journal of Chemical Physics, 131(10):104110, 2009. 
[42] W. G. Noid. Perspective: Coarse-grained models for biomolecular systems. The Journal of Chemical Physics, 139:090901, 2013.

[43] W. G. Noid, J.-W. Chu, G. S. Ayton, V. Krishna, S. Izvekov, G. A. Voth, A. Das, and H. C. Andersen. The multiscale coarse-graining method. I. A rigorous bridge between atomistic and coarse-grained models. The Journal of Chemical Physics, 128:244114, 2008.

[44] W. G. Noid, P. Liu, Y. Wang, J.-W. Chu, G. S. Ayton, S. Izvekov, H. C. Andersen, and G. A. Voth. The multiscale coarse-graining method. II. Numerical implementation for coarse-grained molecular models. The Journal of Chemical Physics, 128:244115, 2008.

[45] J. T. Oden, A. Hawkins, and S. Prudhomme. General diffuse-interface theories and an approach to predictive tumor growth modeling. Mathematical Models and Methods of Applied Science, 20(3):1-41, 2010.

[46] J. T. Oden, R. Moser, and O. Ghattas. Computer predictions with quantified uncertainty, Part I. SIAM News, 43(9), November 2010.

[47] J. T. Oden, R. Moser, and O. Ghattas. Computer predictions with quantified uncertainty, Part II. SIAM News, 43(10), December 2010.

[48] J. T. Oden, E. E. Prudencio, and P. T. Bauman. Virtual model validation of complex multiscale systems: Applications to nonlinear elastostatics. Computer Methods in Applied Mechanics and Engineering, 266:162-184.

[49] J. T. Oden, E. E. Prudencio, and A. Hawkins-Daarud. Selection and assessment of phenomenological models of tumor growth. Mathematical Models and Methods in Applied Sciences, 23(7):1309-1338, 2013.

[50] J. T. Padding and W. J. Briels. Uncrossability constraints in mesoscopic polymer melt simulations: Non-rouse behavior of c120h242. The Journal of Chemical Physics, 115(6):2846-2859, 2001.

[51] J. T. Padding and W. J. Briels. Zero-shear stress relaxation and long time dynamics of a linear polyethylene melt: A test of rouse theory. The Journal of Chemical Physics, 114(19):8685-8693, 2001.

[52] S. Plimpton. Fast parallel algorithms for short-range molecular dynamics. Journal of Computational Physics, 117:1-19, 1995.

[53] E. Prudencio and K. Schulz. The parallel c++ statistical library queso: Quantification of uncertainty for estimation, simulation and optimization. In M. Alexander, P. DAmbra, A. Belloum, G. Bosilca, M. Cannataro, M. Danelutto, B. Martino, M. Gerndt, E. Jeannot, R. Namyst, J. Roman, S. Scott, 
J. Traff, G. Valle, and J. Weidendorfer, editors, Euro-Par 2011: Parallel Processing Workshops, volume 7155 of Lecture Notes in Computer Science, pages 398-407. Springer Berlin Heidelberg, 2012.

[54] D. Reith, M. Pütz, and F. Müller-Plathe. Deriving effective mesoscale potentials from atomistic simulations. The Journal of Computational Chemistry, 24(13):1624-1636, 2003.

[55] A. Saltelli. Making best use of model evaluations to compute sensitivity indices. Computer Physics Communications, 145(2):280 - 297, 2002.

[56] A. Saltelli, P. Annoni, I. Azzini, F. Campolongo, M. Ratto, and S. Tarantola. Variance based sensitivity analysis of model output. design and estimator for the total sensitivity index. Computer Physics Communications, 181(2):259 $270,2010$.

[57] A. Saltelli, K. Chan, and E. Scott. Sensitivity Analysis. Number no. 2008 in Wiley paperback series. Wiley, 2009.

[58] A. Saltelli, M. Ratto, T. Andres, F. Campolongo, J. Cariboni, D. Gatelli, M. Saisana, and S. Tarantola. Global Sensitivity Analysis: The Primer. Wiley, 2008.

[59] A. Saltelli and S. Tarantola. On the relative importance of input factors in mathematical models. Journal of the American Statistical Association, 97(459):702-709, 2002.

[60] M. S. Shell. The relative entropy is fundamental to multiscale and inverse thermodynamic problems. The Journal of Chemical Physics, 129(14):144108, 2008 .

[61] Q. Shi, S. Izvekov, and G. A. Voth. Mixed atomistic and coarse-grained molecular dynamics: Simulation of membrance-bound ion channel. The Journal of Physical Chemistry B, 110(31):15045-15048, 2006.

[62] I. M. Sobol'. Sensitivity estimates for nonlinear mathematical models. Matematicheskoe Modelirovanie, 2:112-118, 1990.

[63] I. M. Sobol'. Sensitivity analysis for non-linear mathematical models. Mathematical Modeling and Computational Experiment, 1:407-414, 1993.

[64] Q. Wang, S. R. Kulkarni, and S. Verdú. Divergence estimation for multidimensional densities via $\mathrm{k}$-nearest neighbor distances. IEEE Transactions on Information Theory, 55(5), May 2009.

[65] P. K. Weiner and P. A. Kollman. AMBER: Assisted Model Building with Energy Refinement. A general program for modeling molecules and their interactions. Journal of Computational Chemistry, 2(3):287-303, 1981. 
[66] J. Zhou, I. F. Thorpe, S. Izvekov, and G. A. Voth. Coarse-grained peptide modeling using a systematic multiscale approach. Biophysical Journal, 92(12):42894303, 2007.

\section{A Sensitivity Analysis}

Sensitivity analysis is the study of how the change in input factors of a model, qualitatively and quantitatively, affects the variation and uncertainty in the model outputs. Among the various existing techniques for sensitivity analysis of model output, variance-based methods $[15,62,63]$ has been proven to be effective and well suited for practical application. In these methods the sensitivity of the output to an input variable is measured by the amount of (conditional) variance in the output caused by that input. Variance-based methods are model independent and address interaction effects among input factors.

\section{A.1 Decomposition of Variance}

Suppose a model $Y=f\left(\theta_{1}, \theta_{2}, \ldots, \theta_{k}\right)$ with $k$ uncertain input factors $\theta_{i}$, where $Y$ is model output and $f$ is a square integrable function over the $k$-dimensional unit hypercube,

$$
\Omega^{k}=\left(\boldsymbol{\theta} \mid 0 \leq \theta_{i} \leq 1 ; i=1, \ldots, k\right) .
$$

Using the Hoeffding decomposition of $f[62,63]$ and conditional expectations of the model, $E\left(Y \mid \theta_{i}\right)$, one can retrieve the following decomposition of the output variance, $V(Y)$,

$$
V(Y)=\sum_{i} V_{i}+\sum_{i} \sum_{j>i} V_{i j}+\cdots+V_{12 \ldots k}
$$

where

$$
\begin{array}{ccc}
V_{i}=V\left(f_{i}\left(\theta_{i}\right)\right)= & V_{\theta_{i}}\left(E_{\boldsymbol{\theta}_{\sim i}}\left(Y \mid \theta_{i}\right)\right), \\
V_{i j}=V\left(f_{i j}\left(\theta_{i}, \theta_{j}\right)\right)= & V_{\theta_{i} \theta_{j}}\left(E_{\boldsymbol{\theta}_{\sim i j}}\left(Y \mid \theta_{i}, \theta_{j}\right)\right) \quad-V_{\theta_{i}}\left(E_{\boldsymbol{\theta}_{\sim i}}\left(Y \mid \theta_{i}\right)\right) \\
& -V_{\theta_{j}}\left(E_{\boldsymbol{\theta}_{\sim j}}\left(Y \mid \theta_{j}\right)\right),
\end{array}
$$

and so on for higher order terms. In (46) $\theta_{i}$ is the $i$-th factor, $\boldsymbol{\theta}_{\sim i}$ denotes the matrix of all factors but $\theta_{i}, V_{\theta_{i}}\left(E_{\boldsymbol{\theta}_{\sim i}}\left(Y \mid \theta_{i}\right)\right)$ and $V_{\theta_{j}}\left(E_{\boldsymbol{\theta}_{\sim j}}\left(Y \mid \theta_{j}\right)\right)$ are the first-order effects, and $V_{\theta_{i} \theta_{j}}\left(E_{\boldsymbol{\theta}_{\sim i j}}\left(Y \mid \theta_{i}, \theta_{j}\right)\right)$ is the joint effect of the pair $\left(\theta_{i} ; \theta_{j}\right)$ on $Y$. In $V_{\theta_{i}}\left(E_{\theta_{\sim i}}\left(Y \mid \theta_{i}\right)\right)$, the inner expectation operator accounts for the mean of $Y$ taken over all possible values of $\boldsymbol{\theta}_{\sim i}$ while keeping $\theta_{i}$ fixed, and the outer variance is taken over all possible values of $\theta_{i}$. Therefore, $V_{\theta_{i}}\left(E_{\boldsymbol{\theta}_{\sim i}}\left(Y \mid \theta_{i}\right)\right)$ is the expected reduction in variance that would be obtained when $\theta_{i}$ is fixed. 


\section{A.2 Sensitivity indices}

For non-additive models (e.g. the molecular models under study), the response of $Y$ depends on the interaction between $\theta_{i}$ and $\theta_{j}$. Dividing (45) by $V(Y)$, forms the sensitivity indices $S_{i}, S_{i j}, \cdots, S_{12 \ldots k}$, yielding

$$
\sum_{i} S_{i}+\sum_{i} \sum_{j>i} S_{i j}+\ldots+S_{12 \ldots k}=1 .
$$

Decomposing the variance by conditioning with respect to all factors but $X_{i}$,

$$
V(Y)=V_{\boldsymbol{\theta}_{\sim i}}\left(E_{\theta_{i}}\left(Y \mid \boldsymbol{\theta}_{\sim i}\right)\right)+E_{\boldsymbol{\theta}_{\sim i}}\left(V_{\theta_{i}}\left(Y \mid \boldsymbol{\theta}_{\sim i}\right)\right) .
$$

and dividing both sides by $V(Y)$, results in a variance-based sensitivity measure, the so-called the total effect index $[23,59]$,

$$
S_{T_{i}}=\frac{E_{\boldsymbol{\theta}_{\sim i}}\left(V_{\theta_{i}}\left(Y \mid \boldsymbol{\theta}_{\sim i}\right)\right)}{V(Y)}=1-\frac{V_{\boldsymbol{\theta}_{\sim i}}\left(E_{\theta_{i}}\left(Y \mid \boldsymbol{\theta}_{\sim i}\right)\right)}{V(Y)},
$$

where $E_{\boldsymbol{\theta}_{\sim i}}\left(V_{X_{i}}\left(Y \mid \boldsymbol{\theta}_{\sim i}\right)\right)$ is the remaining variance of $Y$ for fixed values of $\theta_{i}$ and $V_{\boldsymbol{\theta}_{\sim i}}\left(E_{\theta_{i}}\left(Y \mid \boldsymbol{\theta}_{\sim i}\right)\right)$ is the expected reduction in variance if all values other than $\theta_{i}$ are fixed.

Total effect $S_{T_{i}}$ measures the total contribution to the output variation due to factor $\theta_{i}$. Small values of the total effect index $\left(S_{T_{i}} \approx 0\right)$ imply that $X_{i}$ can be fixed at any value within its range of variability (uncertainty) without appreciably affecting the output. $S_{T_{i}}$ provides information on the non-additive feature of model. Note that since the interaction effect among factors $\theta_{i}$ and $\theta_{j}$ is counted in both $S_{T_{i}}$ and $S_{T_{j}}$, the sum of the $S_{T_{i}}$ will be larger than $1, \sum_{i=1}^{k} S_{T_{i}} \geq 1$.

\section{A.3 Computation of the sensitivity indices}

Saltelli $[55,56,58]$ presented a Monte Carlo scheme to compute the indices with a reduced computational cost of evaluating multi-dimensional integrals. The method is the extension of the original approach proposed by Sobol' $[62,63]$ and Homma and Saltelli [23] with the following procedure:

- Generate two $N \times k$ independent sampling matrices, $\mathbf{A}$ and $\mathbf{B}$, where each row is a sample point in the hyperspace of $k$ dimensions. $N$ is called a base sample with respect to the probability distributions of the input variables.

- Define a matrix $\mathbf{D}_{i}$ formed by all columns of $\mathbf{A}$ except the $i$ th column, which is from $\mathbf{B}$.

- Compute the model outputs for all the matrices (i.e. $N \times 1$ vectors of model outputs):

$$
\mathcal{Y}_{A}=f(\mathbf{A}) \quad ; \quad \mathcal{Y}_{B}=f(\mathbf{B}) \quad ; \quad \mathcal{Y}_{D_{i}}=f\left(\mathbf{D}_{i}\right)
$$


- The total-effect indices can be estimated by,

$$
S_{T_{i}} \approx 1-\frac{\frac{1}{N} \sum_{j=1}^{N} \mathcal{Y}_{A}^{(j)} \mathcal{Y}_{D_{i}}^{(j)}-\left(\frac{1}{N} \sum_{j=1}^{N} \mathcal{Y}_{A}^{(j)}\right)^{2}}{\frac{1}{N} \sum_{j=1}^{N} \mathcal{Y}_{A}^{(j)} \mathcal{Y}_{A}^{(j)}-\left(\frac{1}{N} \sum_{j=1}^{N} \mathcal{Y}_{A}^{(j)}\right)^{2}}
$$

The relation (51) can be obtained by substituting the Monte-Carlo estimators proposed by Saltelli [55],

$$
V_{\boldsymbol{\theta}_{\sim i}}\left[E_{\theta_{i}}\left(Y \mid \boldsymbol{\theta}_{\sim i}\right)\right] \approx \frac{1}{N} \sum_{j=1}^{N} \mathcal{Y}_{A}^{(j)} \mathcal{Y}_{D_{i}}^{(j)}-E^{2}(Y)
$$

along with

$$
V(Y)=E\left(Y^{2}\right)-E^{2}(Y)=\frac{1}{N} \sum_{j=1}^{N} \mathcal{Y}_{A}^{(j)} \mathcal{Y}_{A}^{(j)}-\left(\frac{1}{N} \sum_{j=1}^{N} \mathcal{Y}_{A}^{(j)}\right)^{2}
$$

into (49). 